\title{
ESTABLISHMENT OF THE CENTER FOR ADVANCED SEPARATION TECHNOLOGIES
}

\author{
ANNUAL TECHNICAL PROGRESS REPORT \\ Report Period \\ October 1, 2003 to September 30, 2004
}

Compiled by

Christopher E. Hull

Issued January 30, 2005

DOE Award Number:

DE-FC26-01NT41091

Center for Advanced Separation Technologies

Virginia Polytechnic Institute \& State University

Blacksburg, Virginia 24061-0258

and

National Research Center for Coal and Energy

West Virginia University

Morgantown, WV 26506-6064 


\section{DISCLAIMER}

This report was prepared as an account of work sponsored by an agency of the United States Government. Neither the United States Government nor any agency thereof, nor any of their employees, makes any warranty, express or implied, or assumes any legal liability or responsibility for the accuracy, completeness, or usefulness of any information, apparatus, product, or process disclosed, or represents that its use would not infringe privately owned rights. Reference herein to any specific commercial product, process, or service by trade name, trademark, manufacture, or otherwise does not necessarily constitute or imply its endorsement, recommendation, or favoring by the United States Government or any agency thereof. The views and opinions of authors expressed herein do not necessarily state or reflect those of the United States Government or any agency thereof. 


\begin{abstract}
This Technical Progress Report describes progress made on the eight sub-projects awarded in the first year and the five projects awarded in the second year of Cooperative Agreement DE-FC26-01NT41091: Establishment of the Center for Advanced Separation Technologies. This work is summarized in the body of the main report: the individual subproject Technical Progress Reports are attached as Appendices.
\end{abstract}

Note: SI is an abbreviation for "Le Systeme International d’Unites." 


\section{TABLE OF CONTENTS}

DISCLAIMER

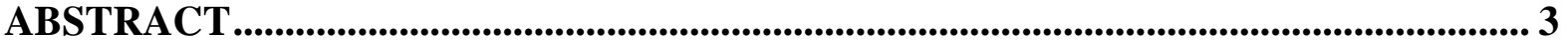

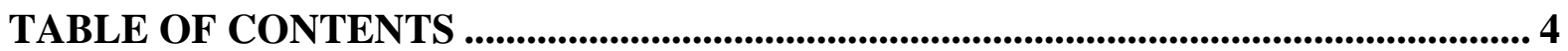

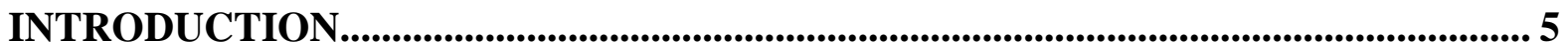

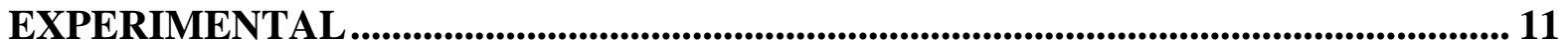

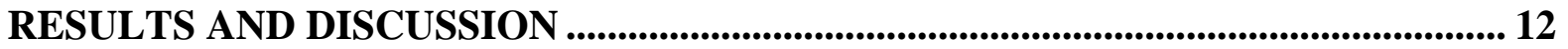

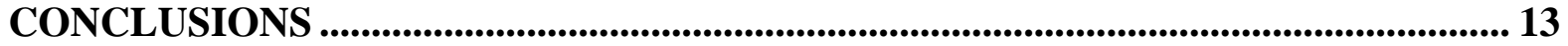

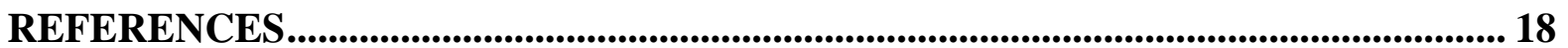

APPENDIX A: IMPROVING COARSE PARTICLE FLOTATION ............................ 19

APPENDIX B: MODELING OF FLOTATION FROM FIRST PRINCIPLES ........... 26 APPENDIX C: STUDIES OF FROTH STABILITY AND MODEL DEVELOPMENT

APPENDIX D: DIRECT MEASUREMENT OF FORCES IN FLOTATION SYSTEMS

APPENDIX E: NOVEL SURFACTANTS AS COLLECTORS FOR FROTH

FLOTATION.

APPENDIX F: COLUMN FLOTATION OF FINE DOLOMITIC PHOSPHATE BY SELECTIVE FATTY ACIDS.

APPENDIX G: DEVELOPMENT OF A NOVEL FINE PARTICLE CENTRIFUGE. 73 APPENDIX H: NEW STRATEGIES FOR DEWATERING OF COALS .................... 81 APPENDIX I: NOVEL BIOLEACHING TECHNOLOGY ASSISTED BY ELECTROLYTIC PROCESSES 86

APPENDIX J: EVALUATION OF COAL CLEANING EFFICIENCY USING TRANSPONDER-BASED DENSITY TRACERS. 90 


\section{INTRODUCTION}

The U.S. is the largest producer of mining products in the world. In 1999, U.S. mining operations produced $\$ 66.7$ billion worth of raw materials that contributed a total of $\$ 533$ billion to the nation's wealth. Despite these contributions, the mining industry has not been well supported with research and development funds as compared to mining industries in other countries. To overcome this problem, the Center for Advanced Separation Technologies (CAST) was established by Virginia Tech and West Virginia University to develop technologies that can be used by the U.S. mining industry to create new products, reduce production costs, and meet environmental regulations. This endeavor is supported through U.S. DOE Cooperative Agreement No. DE-FC26-01NT41091: Establishment of the Center for Advance Separation Technologies.

Much of the research to be carried out at CAST will be longer-term, high-risk, basic research, and will be carried out in four broad areas:

a) Solid-solid separation

b) Solid-liquid separation

c) Chemical/Biological extraction, and

d) Sensor and control development.

Distribution of funds has been handled via competitive solicitation of research proposals through Site Coordinators at the two universities. Two RFP's have been issued to date. The first of these, referred to as the CAST I - Round 1 RFP, was issued on August 17, 2001 and closed October 15, 2001. A total of 12 proposals were received in response to this first RFP. These were first reviewed and ranked by a group of technical reviewers (selected primarily from industry). Based on these reviews, and an assessment of overall program requirements, the CAST Technical Committee made an initial selection/ranking of proposals and forwarded these proposals to the DOE/NETL Project Officer for final review and approval. This process took some 6 months to complete but 8 projects were in place at the constituent universities ( 5 at Virginia Tech and three at West Virginia University) by May 17, 2002.

This was followed, on June 1, 2002 with the CAST I - Round 2 RFP, which closed on July 19, 2002. A total of 12 proposals were received in response to this second RFP five were selected for funding. These were in place at the constituent universities (2 at Virginia Tech and 3 at West Virginia University) by March 1, 2003.

All thirteen of these projects are listed below by category, along with brief abstracts of their aims and objectives. 


\section{a) Solid-Solid Separation}

\section{Improving Coarse Particle Flotation}

Principal Investigators: R.-H. Yoon and G.H. Luttrell, (Virginia Tech).

Period of Performance: May 17, 2002-May 31, 2005.

The recovery of coarse particles by flotation is a common problem in the coal and minerals processing industry. The objective of this project is to develop methods of extending the upper particle size limit for flotation. The availability of such a technology will improve processing efficiency and greatly simplify the plant flowsheet. Two different complementary approaches will be used to achieve the project objective. One is to use novel flotation reagents that will help increase the attachment force between air bubbles and coarse particles, and the other is to develop new flotation machines specifically designed to promote coarse particle flotation.

\section{Separation of Small Particles due to Density Differences in a CFB Riser System} Principal Investigators: E. Johnson and B. Kang (West Virginia University). Period of Performance: May 17, 2002-May 31, 2005.

The efficiency of separating small (less than 250 micrometers in diameter) dense particles from small light particles in the internal recirculation flow within a CFB riser is being investigated. Currently, there are many systems for separating fine particles. However, these systems lack the efficiency and/or the economics to be commercially viable in many situations. This research is directed towards removing pyrite from coal fines at a coal cleaning plant. Also, of concern will be the removal of radioactive particles from the desert sand at the Nevada Test Sites.

\section{Modeling of Flotation From First Principles}

Principal Investigator: R.-H. Yoon (Virginia Tech).

Period of Performance: May 17, 2002-May 31, 2005.

Flotation is widely regarded as the best available technology for separating fine particles. The present form of the technology was invented nearly 100 years ago, yet there is still no reliable model for predicting flotation rate from first principles. It is the purpose of the proposed work to develop a flotation model that can predict flotation performance from both surface chemistry and hydrodynamic parameters.

\section{Studies of Froth Stability and Model Development}

Principal Investigator: R.-H. Yoon (Virginia Tech).

Period of Performance: May 17, 2002-May 31, 2005.

Froth plays an important role in flotation. It determines the final grade of the product and the maximum carrying capacity (or throughput) of a flotation machine. Also, many operators use stronger frothers to produce smaller air bubbles and, hence, higher recovery and throughput. Despite its importance, little is known of the fundamentals of foam and froth stability. It is, therefore, proposed to study the various factors affecting the stability 
of flotation froth. This will be accomplished by using a thin film balance technique and by monitoring the stability of froth in a bubble column using a video camera. The results will be used for developing a froth model and also for developing effective defoamers.

\section{Direct Measurement of Forces in Flotation Systems}

Principal Investigators: W.A. Ducker and R.-H. Yoon (Virginia Tech).

Period of Performance: May 17, 2002-May 31, 2005.

The objective of this project is to directly measure the force as a function of separation between a particle and a bubble in aqueous solution. This force controls the attachment and detachment of particles to bubbles, which is an essential step in determining the efficiency of the separation of mineral particles in a flotation cell. We are fabricating a device specially designed for these measurements. The device will use a force-detection method from an Atomic Force Microscope and a separation-detection method from a Surface Forces Apparatus. The key advances are to explicitly measure both the separation between the particle and the bubble and the shape of the bubble at all times. Without this separation and shape data, it is not possible to compare the measured forces to theoretical estimates or to the practice of flotation. After fabrication of the device, measurements of the forces acting on hydrophilic, hydrophobic, and charged particles in aqueous solutions of surfactant molecules will be obtained.

\section{Novel Surfactants as Collectors for Froth Flotation}

Researcher: Richard D. Gandour (Virginia Tech)

Period of Performance: March 1, 2003-May 31, 2005.

Our goals are to synthesize novel, inexpensive surfactants that outperform current collectors in mineral flotation. These surfactants will selectively bind to particles and create a hydrophobic coating on a particle to enable selective separation by froth flotation. We plan to synthesize surfactants that have very hydrophobic, long chains, yet have sufficient water solubility for mineral processing and coal-fines processing. These amphiphiles, which we call "hydra surfactants," will have one tail and three identical functional groups on the head. These novel molecules will be useful for many different separations in mineral processing, but initially will be tested on separation of discoloring impurities (i.e., anatase (TiO2) and iron oxides) from clay.A strategy to make these surfactants from environmentally friendly vegetable oils is outlined.

\section{Column Flotation of Fine Dolomitic Phosphate by Selective Fatty Acids}

Researcher: F. F. Peng (West Virginia University)

Period of Performance: March 1, 2003-May 31, 2005.

Among the detrimental impurities in phosphate ores, dolomite is considered the most troublesome for down stream operations. Dolomite may cause higher consumption of sulfuric acid, reduce filtration rates and lower $\mathrm{P}_{2} \mathrm{O}_{5}$ content in fertilizer manufacturing processes. Difficulties in separating dolomite from phosphate arise from the fact that most of the dolomite is disseminated in the phosphate mineral. Both are oxide type minerals and have the same calcium cationic component. They exhibit similar 
electrokinetics, adsorption and desorption behavior in physical separation process such as flotation. Several processing schemes have been developed to handle this problem, but none are satisfactory due to high $\mathrm{MgO}$ content and/or low overall $\mathrm{P}_{2} \mathrm{O}_{5}$ recovery in the final phosphate concentrate. In this proposed research project, samples from Florida dolomitic phosphate minerals will be tested in an open flotation column. New selective fatty acid collectors for dolomite particles and appropriate mixtures of phosphoric acid/sulfuric acid depressant for phosphate particles will be selected and used. The aim is to achieve a phosphate concentrate containing as high as $30 \% \mathrm{P}_{2} \mathrm{O}_{5}$ and less than $1.0 \%$ $\mathrm{MgO}$ contents at high $\mathrm{P}_{2} \mathrm{O}_{5}$ recovery. Liberation analysis of dolomitic phosphate pebble will be conducted. The selectivity and frothability of the new selective fatty acid collectors will also be characterized by determining dolomitic phosphate flotation. Success in this research project will enable the mineral industry to increase its phosphate reserve base, by recovering phosphate from formerly unusable phosphatic minerals and low grade phosphate reserves.

\section{b) Solid-Liquid Separations}

\section{Development of a Novel Fine Particle Centrifuge}

Researchers: G.H. Luttrell and R-H. Yoon (Virginia Tech)

Period of Performance: March 1, 2003-May 31, 2005.

The solid-solid separation processes employed by modern coal preparation plants require large amounts of process water. After cleaning, the unwanted water must be removed from the surfaces of the particles using mechanical dewatering equipment. Coarse particles can be readily dewatered using simple screening systems, while finer particles require more complicated unit operations such as centrifuges and filters. Unfortunately, the processes used to dewater fine particles are inherently inefficient and expensive to operate and maintain. To overcome these problems, a novel centrifugal filter has been developed by researchers at Virginia Tech. Preliminary test data suggest that this new technology can reduce the moisture content of fine coal products by approximately $30-50 \%$ compared to existing dewatering processes. The objective of this project will be to construct a continuous prototype unit and to conduct a detailed experimental investigation of this new technology.

\section{New Strategies for Dewatering of Coals}

Researchers: M. Seehra, A. Manivannan \& M. Bachlechner (West Virginia University)

Period of Performance: March 1, 2003-May 31, 2005.

A two-year innovative research program on the combined experimental-modeling studies of coal-water interactions and coal dewatering is proposed. The experimental program will first focus on determining the relative amounts of "free" and "bound" water in wet coals using analytical techniques of thermogravimetric analysis, heat capacity measurements and $1 \mathrm{H}$ nuclear magnetic resonance. Laboratory scale dewatering experiments will then be carried out by vacuum filtration and centrifugation, combined with pulsed heating at microwave or IR $(3300 \mathrm{~cm}-\mathrm{l})$ frequencies to liberate the "bound" water. These investigations will be complimented with modeling studies of the coal-water 
interactions and the effect of centrifugation on dewatering from surface-bound water and coal pores. This integrated experimental-modeling approach is an important component of the proposed research since the results from the molecular-dynamics simulations could provide unique technological insights that will increase the efficiency of dewatering processes. Finally, scale-up of the resulting successful approaches for coal dewatering will be proposed at the conclusion of the two-year program.

\section{c) Chemical/Biological Extraction}

\section{Novel Bioleaching Technology Assisted by Electrolytic Processes}

Principal Investigators: E. Cho and R. Yang (West Virginia University).

Period of Performance: May 17, 2002-May 31, 2005.

Bioleaching of sulfide minerals using bacteria Thiobacillus ferrooxidans has been identified as a promising method for heap or dump leaching of low grade mixed sulfide ores containing pyrite $\left(\mathrm{FeS}_{2}\right)$, chalcopyrite $\left(\mathrm{CuFeS}_{2}\right)$, and/or sphalerite ( $\left.\mathrm{ZnS}\right)$. However, the major problem with bioleaching is that the rates are too slow for wide commercial utilization. The approach being used in this project focuses on the growth of bacteria under the influence of an applied direct potential and on the utilization of the bacteria to leach sulfide minerals. The objective is to identify the optimum conditions for the bioand electro- leaching reactions in order to maximize overall leaching rates of the sulfide minerals.

\section{Coal Desulfurization Using Hypochlorite and Cupric Ion as a Catalyst - Feasibility Study}

Researchers: E. H. Cho \& R. Y. K. Yang (West Virginia University)

Period of Performance: March 1, 2003-May 31, 2005.

The utilization of coal as a feedstock to produce carbon materials is very much hampered by the presence of its organic sulfur content. However, the organic sulfur is very difficult to remove. It is generally accepted that only two methods have capability to remove the organic sulfur rather effectively; one is with a melt of sodium hydroxide/potassium hydroxide at high temperatures (Gravimelt Process) and the other is with chlorine chemicals. However, the Gravimelt Process contaminates the coal structure, and its decontamination and the regeneration of the reagents may be insurmountable problems in terms of economic feasibility. Chlorine chemicals also chlorinate the coal structure and the removal of organic sulfur is not high enough to produce a premium coal or feedstock. It is proposed that organic sulfur be leached with hypochlorite using cupric amine as a catalyst. Hypochlorite is the predominant chlorine derivative at high pH's. The catalysis of cupric amine was found during 1990s in leaching of gold with hypochlorite and other oxidants. We will conduct two kinds of consecutive experiments. In the first, coal will be leached in a reactor with a solution containing hypochlorite and cupric amine. In the second, the leached coal will be hydrolyzed in hot sodium carbonate solution, which is believed to cleave the organic sulfur bond from coal and thus further reduce the sulfur content. Leached coal and hydrolyzed coal samples will be analyzed particularly for organic sulfur and chloride content. The data will be analyzed to 
determine the desulfurization potential through the two consecutive experiments and also the chlorination/dechlorination of the coal matrix through each of the two experiments.

\section{d) Sensor and Control Development}

\section{Development of Electrochemical Sensor for On-Site Monitoring of Heavy Metal Ions in Coal Processing And Utilization}

Principal Investigators: A. Manivannan and M. Seehra (West Virginia University). Period of Performance: May 17, 2002-May 31, 2005.

The aim of this research program is to develop a novel electrochemical sensor based on a boron-doped diamond (BDD) electrode for monitoring/controlling heavy metal ions such as $\mathrm{Hg}, \mathrm{Zn}, \mathrm{Cu}, \mathrm{Pb}, \mathrm{As}, \mathrm{Cd}$, and $\mathrm{Fe}$ encountered in the processing and utilization of coals. This research is based on earlier testwork in which ppb levels of $\mathrm{Pb}$ and Hg were detected in laboratory prepared solutions. BDD electrodes are superior to other commonly used electrodes (such as glassy carbon) in terms of their ruggedness, chemical stability, wide potential window and lower background current. These advantages are important for the simultaneous detection of a number of elements in solution.

\section{Evaluation of Coal Cleaning Efficiency Using Transponder-Based Density Tracers} Principal Investigators: G.H. Luttrell, R.-H. Yoon and C.J. Wood (Virginia Tech). Period of Performance: May 17, 2002-May 31, 2005.

Density tracers are one of the most powerful diagnostic tools for evaluating the performance of heavy media circuits in coal preparation plants. The objective of this project is to develop a new generation of tracers that can be automatically tracked using recently developed transponder technology. This development also makes it possible for efficiency tests to be performed very rapidly by a single person in an extremely cost-efficient manner. The project will be carried out in two phases. In the first phase of work, the hardware and software required to develop a prototype transponder-based system will be procured and evaluated using a simulated circuit at the Virginia Tech coal laboratory. In the second phase, the monitoring system will be relocated to an industrial coal preparation plant for field-testing. 


\section{EXPERIMENTAL}

The CAST initiative is comprised of a diverse group of subprojects, most of which are multistage, task-oriented developmental projects that cannot be conveniently categorized by the traditional reporting criteria required by the DOE Uniform Reporting Requirements. For example, several of the projects have required the construction of unique test equipment, others the generation of simulation models, etc., as preliminary tasks in the overall execution of the project. As such, they are more appropriately described and discussed as "Project Tasks” within the context of the individual Technical Progress Reports. These reports are attached to this document as Appendices and should be referred to for this information. 


\section{RESULTS AND DISCUSSION}

The CAST initiative is comprised of a diverse group of subprojects, most of which are multistage, task-oriented developmental projects that cannot be conveniently categorized by the traditional reporting criteria required by the DOE Uniform Reporting Requirements. For example, several of the projects have required the construction of unique test equipment, others the generation of simulation models, etc., as preliminary tasks in the overall execution of the project. As such, the presentation of results is more appropriately described and discussed within the context of the individual Technical Progress Reports. These reports are attached to this document as Appendices and should be referred to for this information. 


\section{CONCLUSIONS}

This aggregated Technical Progress Report covers the third year of operation of Cooperative Agreement DE-FC26-01NT41091: Establishment of the Center for Advanced Separation Technologies. A total of thirteen (13) sub-projects are currently funded: twelvemonthly Progress Reports (covering the period October 1, 2003-September, 2004) have been generated for 10 of these projects which are still in progress. These are attached to this document as Appendices. Three (3) projects have been completed and final reports will be issued separately. A brief summary of progress during the year, along with plans for the future, is given below for each of the sub-projects.

\section{a) Solid-Solid Separation}

\section{VA003 - Improving Coarse Particle Flotation (Appendix A)}

Principal Investigators: R.-H. Yoon and G.H. Luttrell (Virginia Tech)

Period of Performance: May 17, 2002-May 31, 2005

Direct measurements of the bubble-particle detachment force were made during this reporting period. The experiments carried out to date indicate that the detachment of a hydrophobic glass sphere from the bubble occurs as two dependent events. The energy required in the first stage (E1) is dependent on the chemical properties of the bulk solution (i.e., surface tension), while the energy required in the second stage (E2) is dependent on the particle hydrophobicity. The detachment energy calculated from these measurements was successfully correlated with the theoretical work of adhesion.

During the next reporting period, additional analyses will be performed using the data obtained from the detachment force measurements. Laboratory testing of several of the novel flotation reagents will also be carried out using relatively coarse samples from various sites. Operating parameters, such as the solids content, agitation speed, $\mathrm{pH}$ levels, and conditioning time, will also be evaluated in greater detail.

\section{WV002 - Separation of Small Particles due to Density Differences in a CFB Riser System}

Principal Investigators: E. Johnson and B. Kang (West Virginia University)

Period of Performance: May 17, 2002-May 31, 2005

A Final Report is to be issued

3. VA001 - Modeling of Flotation From First Principles (Appendix B)

Principal Investigator: R.-H. Yoon (Virginia Tech)

Period of Performance: May 17, 2002-May 31, 2005

A flotation model was developed that can predict flotation from both hydrodynamic and surface chemistry parameters under turbulent flow conditions. It was developed by considering the subprocesses of collision, attachment, detachment, and froth phase 
recovery. The collision subprocess was based on Abrahamson's model, which assumes a Stokes number of infinity. The attachment subprocess was modeled by considering the energy barriers relative to the kinetic energies involved. The energy barriers were determined by considering the surface forces, while the kinetic energies were calculated using the particle velocities at the critical rupture thickness of the wetting film. The detachment subprocess was modeled from the work of adhesion and kinetic energies of detachment. The kinetic energies were calculated by assuming a two-compartment energy dissipation model. The froth recoveries were calculated using the Gorain et al.'s model.

The model was used to simulate flotation using both hydrodynamic and surface chemistry parameters under turbulent conditions. The simulation results show that flotation is efficient at a relatively narrow range of particle sizes. It is also shown that floatation rate is critically dependent on contact angle, zeta-potential and surface tension, as has been amply demonstrated in the literature and in flotation practice.

Further refinement of the flotation model will continue during the next reporting period. This will include a more in-depth theoretical analysis of the effects of turbulence on particle and bubble movements. Experimental work will include the flotation tests to validate the model as well as DPIV data analysis. Also, the froth model presented here will be modified so that it considers the effect of particle size.

\section{VA002 - Studies of Froth Stability and Model Development (Appendix C) \\ Principal Investigator: R.-H. Yoon (Virginia Tech) \\ Period of Performance: May 17, 2002-May 31, 2005}

The pressure decay method was evaluated for studying the stability of foams and froths at low surfactant concentrations. It involves monitoring the change in pressure external to the foam in container of constant volume and temperature. The experimental data are then converted to decay of the interfacial area of the foam as a function of time. The measured results agree well with previous measurements of disjoining pressure isotherm, as well as those obtained using shake test. This technique is thus expected to be useful for studying the effects of particle size, shape and hydrophobicity on froth stability.

Future work will use pressure decay method to investigate froth stability.

\section{VA004 - Direct Measurement of Forces in Flotation Systems (Appendix D) Principal Investigators: W.A. Ducker and R.-H. Yoon (Virginia Tech) Period of Performance: May 17, 2002-May 31, 2005}

During the past reporting period, experimental measurements of surface forces were performed using model (silica) surfaces treated with surfactant (C18TACl). The surfactant produces attractive forces between silica-like surfaces in aqueous solutions. These attractive forces are much greater in magnitude and range than expected for the 
van der Waals force between silica or hydrocarbon surfaces. Both the magnitude and range of the anomalous attractive forces are diminished through the addition of low concentrations of salt (10-4 M NaCl), but are not largely influenced by degassing or $\mathrm{pH}$ changes. In the absence of a good reason to believe that either the adsorption of bubbles or the water structure is dramatically affected by such low concentrations of simple salt or small changes in $\mathrm{pH}$, several other possibilities have been considered to explain the long-range attraction. An explanation that is consistent with the experimental results is that the attractive force is due to correlations between patches of positive charge on one surface and patches of negative charges on the other surface. Such patches could exist on isolated surfaces and the correlations be produced by surface mobility, or patches of opposite charge on each surface could be produced by the fusion and rupture of layers when the surfaces are brought into contact and then separated.

Much of the work conducted during the next reporting period will focus on the continued construction and shakedown testing of the experimental apparatus for the direct measurement of force and separation distance between bubbles and particles.

\section{VA007 - Novel Surfactants as Collectors for Froth Flotation (Appendix E)}

Researcher: Richard D. Gandour (Virginia Tech)

Period of Performance: March 1, 2003-May 31, 2005

A procedure has been developed for making three classes of hydra surfactants which are soluble in aqueous triethanolamine.

Future work will be the scale up of these syntheses to provide samples for testing by Professor Yoon's group.

\section{WV006 - Column Flotation of Fine Dolomitic Phosphate by Selective Fatty Acids (Appendix F)}

Researcher: F. F. Peng (West Virginia University)

Period of Performance: March 1, 2003-May 31, 2005

Experiment runs using flotation column for fine dolomitic phosphate pebble flotation based on Center Composite Experiment Design have been conducted in systematic tests. The results show that the $\mathrm{P} 2 \mathrm{O} 5$ recovery is very high (over 95\%), but $\mathrm{MgO}$ rejection is low due to possible insufficient of collector dosages for this period of the experiment design runs, which can be observed from the appearance and thickness of froth layers. The improvement in reduction of $\mathrm{MgO}$ is expecting for the rest of the tests for the experiment design runs which required more higher dosages.

Experiment design runs for fine dolomitic phosphate pebble column flotation based on Center Composite Experiment Design for systematic testing will be continued in systematic approach, and to complete experiment design runs. The results will be subject to analyzed for ANOVA using JMP software tools for P2O5 recovery and MgO rejection as the responses. 


\section{e) Solid-Liquid Separations}

\section{VA006 - Development of a Novel Fine Particle Centrifuge (Appendix G)}

Researchers: G.H. Luttrell and R-H. Yoon (Virginia Tech)

Period of Performance: March 1, 2003-May 31, 2005

A project has been initiated to construct and test a hyperbaric filter centrifuge that is capable of substantially improving the removal of moisture from fine particle streams. Laboratory experiments performed using a batch laboratory unit indicates that moisture reductions of up to 50\% can be achieved using this new technology. During this reporting period, work was continued to complete the construction of a continuous prototype. The prototype system is comprised of a two-stage process incorporating a thickening step and dewatering step. Once construction is completed, the prototype unit will be used (i) to demonstrate the capabilities of this new technology at a larger scale and (ii) to obtain scale-up data for the engineering design of a production scale unit.

The next step in this project is to complete fabrication of the hyperbaric centrifuge and begin testing as described under Task 2. Also, laboratory-scale tests will run concurrently using the lab centrifuge to further understand and optimize each operating parameter in this novel centrifugation process.

\section{WV004 - New Strategies for Dewatering of Coals (Appendix H)}

Researchers: M. Seehra, A. Manivannan \& M. Bachlechner (West Virginia University)

Period of Performance: March 1, 2003-May 31, 2005

During this reporting period, efforts were directed at designing and constructing a laboratory-scale coal dewatering apparatus using the conventional $2.45 \mathrm{GHz}$ microwaves.

A commercial microwave oven was modified so that wet coal can be fed to a microwave oven on a conveyor belt. This open design necessitated substantial microwave shielding and a remote control electronic circuit. Details of the systems are presented in this report, along with some preliminary results. Also included is a description of the status of the modeling studies of coal-water interactions.

As outlined in the report the end of section (a) under Project Tasks, systematic studies of the efficiency of the microwave dewatering using the laboratory scale unit (Fig. 1 and Fig. 2) are planned for the coming months. The belt speed and hence the optimum resident time of coal in the microwave unit, for maximum dewatering, needs to be determined. The modeling studies should provide some insight into the mechanism of water absorption in coals. 


\section{b) Chemical/Biological Extraction}

10. WV001 - Novel Bioleaching Technology Assisted by Electrolytic Processes (Appendix I)

Principal Investigators: E. Cho and R. Yang (West Virginia University)

Period of Performance: May 17, 2002-May 31, 2005

The leaching conversion of chalcopyrite sample in bioleaching increased as time went on, reached $0.57 \%$ at 22 days and then tended to level off. However, the leaching conversion of the mineral in the electro-bioleaching reached only $0.45 \%$ at the same time period of 22 days but increased without leveling off. It reached $0.64 \%$ in 38 days. These results show that the electro-bioleaching is slightly better than the bioleaching; however, the effect of the electro-bioleaching is not clearly pronounced.

Future experimental work will be focused on the leaching of pyrite and sphalerite in the two leaching modes of bioleaching and electro-bioleaching.

11. WV005 - Coal Desulfurization Using Hypochlorite and Cupric Ion as a Catalyst Feasibility Study

Researchers: E. H. Cho \& R. Y. K. Yang (West Virginia University)

Period of Performance: March 1, 2003-May 31, 2005

A Final Report is to be issued

c) Sensor and Control Development

12. WV003 - Development of Electrochemical Sensor for On-Site Monitoring of Heavy Metal Ions in Coal Processing And Utilization (Final Report to be issued)

Principal Investigators: A. Manivannan and M. Seehra (West Virginia University)

Period of Performance: May 17, 2002-May 31, 2004 (2-Year Project)

A Final Report is to be issued.

13. VA005 - Evaluation of Coal Cleaning Efficiency Using Transponder-Based Density Tracers (Appendix $\mathbf{J}$ )

Principal Investigators: G.H. Luttrell, R.-H. Yoon and C.J. Wood

Period of Performance: May 17, 2002-May 31, 2005

Several important activities were completed during the past reporting period. Prototype blocks were manufactured and were found to have a density tolerance that is acceptable for the proposed work (i.e., +0.01 SG units). The change in block density resulting from the loss of volume due to abrasion was also deemed to be within an acceptable range. Pilot-scale tests conducted using twin conveyor belts to simulate an industrial setting indicated that the low-cost TI RFID system is well suited for this 
particular application. Data obtained from the pilot-scale trials were used to develop an antenna array that can accurately identify tracer tags at a distance of 45-60 cm (18-24 inches) regardless of orientation on a moving conveyor belt.

During the next reporting period, work is expected to focus on (i) production of a large batch of tracers for testing in an industrial application, (ii) the investigation of different antenna configurations/arrays to extend the capabilities of the system, (iii) continued development of data acquisition system for deployment in industrial environment, and (iv) full-scale field testing at an industrial site.

\section{REFERENCES}

References utilized by the individual sub-projects are reported in the relevant Technical Progress Report in the attached Appendices. 
Appendix A: Improving Coarse Particle Flotation 


\section{TECHNICAL PROGRESS REPORT}

\section{Contract Title and Number: \\ Establishment of the Center for Advanced Separation \\ Technologies (DE-FC26-01NT41091)}

Sub-Recipient Project Title:

Improving Coarse Particle Flotation

Principal Investigators:

Roe-Hoan Yoon and Gerald H. Luttrell

Contact Address:

146 Holden Hall

Virginia Polytechnic Institute \& State University

Blacksburg, VA 24061

Subcontractor Address:

No subcontracts issued.
Period of Performance:

Starting Date: 9/17/01

Ending Date: 5/31/05

\section{ABSTRACT}

The recovery of coal particles by flotation is a common problem in the coal and minerals processing industry. The objective of this project is to develop methods of extending the upper particle size limit for flotation. The availability of such a technology will improve processing efficiency and greatly simplify the plant flowsheet. Two different complementary approaches will be used to achieve the project objective. One is to use novel coarse coal floatation reagents that will help increase the attachment force between air bubbles and coal particles, and the other is to develop a new flotation machine specifically designed to promote coarse particle flotation. During this reporting period, fundamental experiments were conducted using a model system in order to develop a better understanding of the bubble-particle detachment mechanism.

\section{INTRODUCTION}

\section{$\underline{\text { Background }}$}

There has been a substantial advancement in the science of flotation during the last two decades. This fundamental research has shown that the probability of bubble-particle attachment can be expressed as: 


$$
\mathrm{P}=\mathrm{P}_{\mathrm{c}} \mathrm{P}_{\mathrm{a}}\left(1-\mathrm{P}_{\mathrm{d}}\right)
$$

in which $P_{c}$ is the probability of collision, $P_{a}$ the probability of bubble-particle adhesion after collision, and $P_{d}$ is the probability of detachment after adhesion. Past investigations suggest that the difficulty in floating coarser particles can be attributed to a large increase in $P_{d}$ as particle size increases. Unfortunately, the underlying factors that control $P_{c}$ are not well understood.

\section{Objective and Approach}

The objective of this project will be to develop two complementary methods for improving the recovery of coarse particles during froth flotation. One method is to use novel coarse flotation reagents that increase the adhesion force between air bubbles and hydrophobic particles. The second method is to develop a flotation machine specifically designed to promote the flotation recovery of coarse particles.

\section{PROJECT TASKS}

\section{Task 1 - Sample Acquisition}

Several samples were collected during the past reporting period for use in Task 4 Batch Flotation Tests. These experiments are currently ongoing and the data will be presented in the next technical progress report.

\section{Task 2 - Detachment Measurements}

Work conducted during the past reporting period primarily focused on measurements of bubble-particle detachment forces. The detachment force was measured using a hanging balance (Sigma 70 surface tensiometer) with a resolution of $1 \mu \mathrm{N}$. A schematic of this deviced was provided in the last technical progress report. The balance was connected to a computer to record force and distances every $0.25 \mathrm{sec}$. The bubble-particle interactions were also visually recorded using a Phantom V4.0 CCD camera. The detachment experiments were conducted by pulling a soda lime glass sphere (mean diameter of $2007 \mu \mathrm{m} \pm 40 \mu \mathrm{m}$ ) from the surface of a flat bubble. The glass sphere was methylated to achieve varying degrees of hydrophobicity. For comparison, advancing and receding contact angles were measured on methylated silica glass plates using the Wilhelmy plate principle. The measured contact angles were used to determine the surface free energy $\left(\gamma_{s}\right)$ using the expression:

$$
\gamma_{s}=\gamma_{l}\left(\cos \theta_{r}-\cos \theta_{a}\right) \frac{\left(1+\cos \theta_{a}\right)^{2}}{\left(1+\cos \theta_{r}\right)^{2}-\left(1+\cos \theta_{a}\right)^{2}}
$$

where, $\gamma_{l}$ is the surface tension of water and $\theta_{r}$ and $\theta_{a}$ are the receding and advancing contact angles, respectively (Chibowski, 2003). 
A typical force curve obtained using the hanging balance is shown in Figure 1. The detachment force is calculated from the difference between the maximum force and the baseline. The baseline is the force exerted by the weight of the sphere only. The energy ( $\mathrm{E}$ ) is taken as the integral of the force $(\mathrm{F})$ applied across the distance between equilibrium and detachment. The equilibrium distance is shown in the figure as $\mathrm{x}_{0}$. This value corresponds to the point at which the bubble is neither pushing up nor pulling down on the glass sphere.

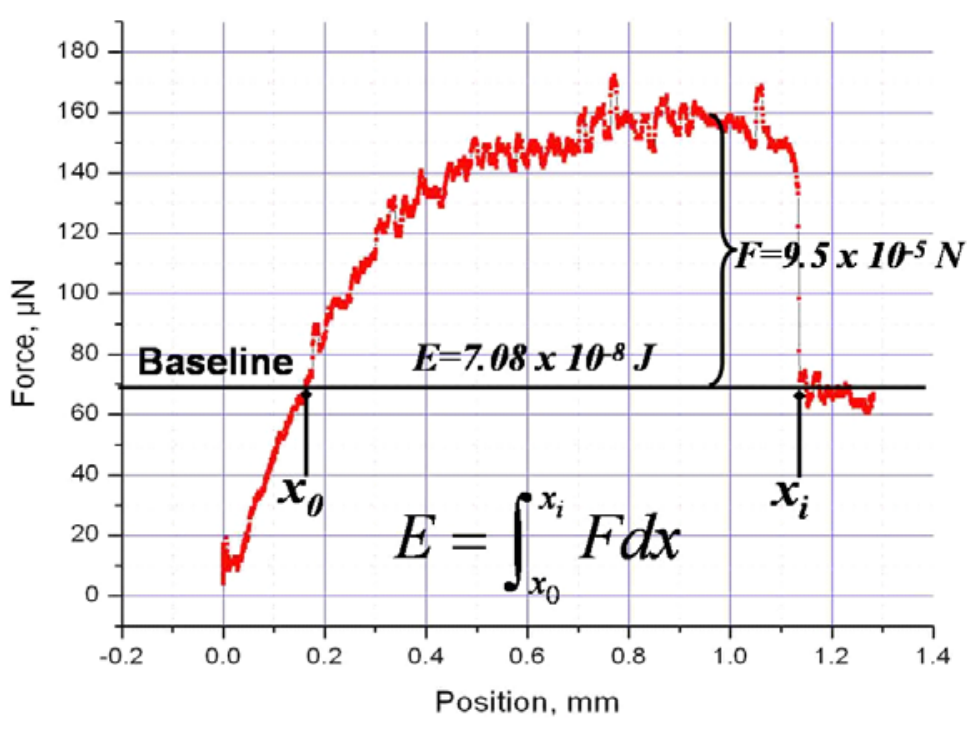

Figure 1. Typical detachment force curve.

The experiments carried out to date indicate that the detachment of a hydrophobic glass sphere from the bubble occurs as two dependent events (Figure 2). The energy required in the first event $\left(E_{1}\right)$ is dependent on the chemical properties of the bulk solution (i.e., surface tension), while the energy required in the second event $\left(E_{2}\right)$ is dependent on the particle hydrophobicity. In the first event, the three-phase-contact (TPC) line is pinned on the silica surface as the bubble is being stretched. Contact area remains constant and the bubble continues to stretch until enough force is applied to move the TPC line up to the advancing contact angle (although surface roughness can cause the bubble to stretch slightly beyond this point). Once the advancing contact angle is reached, the vapour phase begins to retract across the surface of the particle. The force remains constant as the TPC line moves across the sphere until detachment takes place.

Further support for the two-event detachment process can be obtained by comparing the energy components with the work of adhesion. The

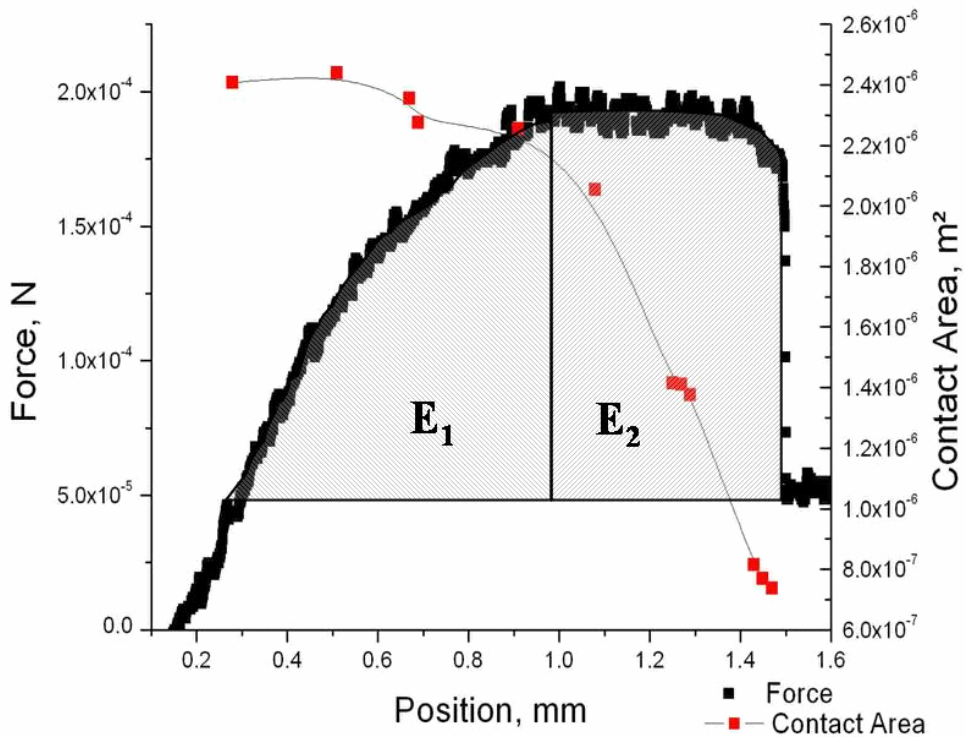

Figure 2. Detachment curve and contact area. 
work of adhesion $\left(\mathrm{W}_{\mathrm{A}}\right)$ can be calculated from differences in surfaces areas of the various interfaces between the initial attached and final detached states. As can be seen from Table 1 , the energy $E_{l}$ is fairly constant for $48^{\circ}$ and $95^{\circ}$ surfaces, because this portion of the detachment does not depend on the surface chemistry properties of the sphere. In mineral processing, this part of the detachment energy could be maximized using frother to create stronger bubbles. $E_{1}$ can also be regarded as the activation energy for detachment due to surface roughness and the magnitude dependent on the surface tension of the bulk solution in which the process takes place. $E_{2}$ shows good correlation with the work of adhesion calculation because it is the actual energy of detachment, whereas $E_{l}$ is the energy necessary to begin (activate) the detachment process.

A mathematical model of the detachment can now be developed. Figure 3 shows the bubble deformation geometry as the glass sphere is pulled away from the bubble surface. The air-water interface deformation can be described by the Huh and Scriven (1969) solution to the Young-Laplace capillary equation. This equation can be solved numerically to determine the height $(h)$ of the TPC line above the flat bubble:

$$
\begin{aligned}
& h=R \sin \alpha \sin \beta\left[\ln \frac{4 L}{R \sin \alpha(1+\cos \beta)}-\sigma\right] \\
& L \equiv \sqrt{\frac{\gamma}{\rho g}}
\end{aligned}
$$

where $\rho$ is the density of the liquid, $g$ is gravity, and $\gamma$ is the interfacial tension, $R$ is the radius of the sphere, $L$ is the capillary length, and $\sigma$ is the Euler constant (James, 1974). The force exerted by the bubble onto the sphere depends on the contact angle and shape of the bubble as these determine the angle at which the force is applied. The total force $F$ exerted on the sphere in the vertical direction is given by:

$$
F=2 \pi R \sin \alpha \cdot \gamma_{L V} \cos \left(\frac{\pi}{2}-\theta+\alpha\right)
$$


When the sphere is at the equilibrium position, the bubble is not deformed and is not exerting any force on the sphere. The angle between the sphere and bubble at this point is the receding contact angle (Preuss and Butt, 1998), which can easily be calculated from the contact radius. Likewise, as the sphere is

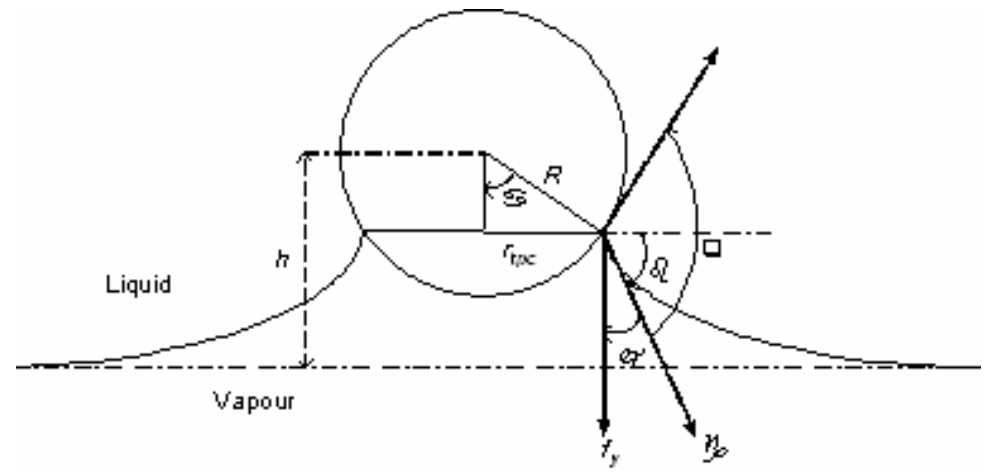

Figure 3. Bubble-sphere detachment geometry. pulled away, the contact angle can be calculated by solving Eq. [3] at progressing heights of the sphere above the bubble. Once the contact angle is known, and assuming a constant contact radius during the stretching portion of the detachment process, the force is calculated using Eq. [4] for each sphere height above the flat bubble datum. Force distance curves obtained in this manner were compared to the measured force curves shown in Figure 4. The "Calculated Force I" represents the stretching portion of the force curve calculated as described above. This term represents the force exerted by the bubble on the sphere as the contact angle shifts from a receding to an advancing contact angle. The "Calculated Force I" shows good correlation with the measured force curve, although Figure 4(a) demonstrates some of the difficulty in simulating the detachment process. The experimental and calculated forces are in agreement for the first third of the curve, at which point the bubble seems to have 'slipped' on the sphere, causing a change in the slope due to the new contact radius.
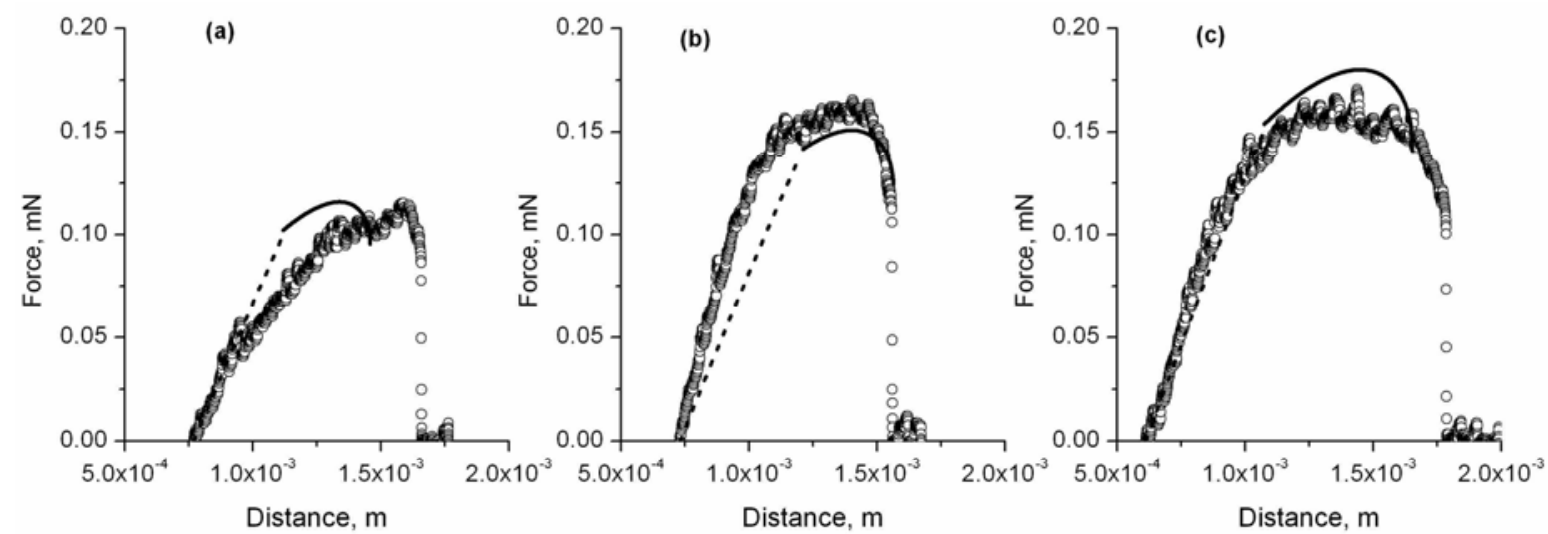

- Measured Force Calculated Force I Calculated Force II

Figure 4. Detachment force vs. sphere center distance from the flat bubble for (a) $38^{\circ}$ (b) $78^{\circ}$ (c) $95^{\circ}$ methylated silica spheres. 


\section{SUMMARY}

Direct measurements of the bubble-particle detachment force were made during this reporting period. The experiments carried out to date indicate that the detachment of a hydrophobic glass sphere from the bubble occurs as two dependent events. The energy required in the first stage $\left(E_{1}\right)$ is dependent on the chemical properties of the bulk solution (i.e., surface tension), while the energy required in the second stage $\left(E_{2}\right)$ is dependent on the particle hydrophobicity. The detachment energy calculated from these measurements was successfully correlated with the theoretical work of adhesion.

\section{FUTURE WORK}

During the next reporting period, additional analyses will be performed using the data obtained from the detachment force measurements. Laboratory testing of several of the novel flotation reagents will also be carried out using relatively coarse samples from various sites. Operating parameters, such as the solids content, agitation speed, $\mathrm{pH}$ levels, and conditioning time, will also be evaluated in greater detail.

\section{REFERENCES}

Chibowski, E. (2003). "Surface free energy of a solid from contact angle hysteresis." Advances in Colloid and Interface Science 103(2): 149-172.

Huh, C. and L. E. Scriven (1969). Shapes of axysimmetric fluid interfaces of unbounded extent. Journal of Colloid and Interface Science. 35: 323.

James, D. F. (1974). The meniscus on the outside of a small circular cylinder. Journal of Fluid Mechanics. 63: 657-664.

Preuss, M. and H.-J. Butt (1998). "Measuring the Contact Angle of Individual Colloidal Particles." Journal of Colloid and Interface Science 208(2): 468-477.

\section{PUBLICATIONS/PRESENTATIONS}

To date, no major publications have resulted from this project.

\section{APPENDICES}

No appendices are included in this report. 
Appendix B: Modeling of Flotation From First Principles 


\section{TECHNICAL PROGRESS REPORT}

\begin{tabular}{|c|c|}
\hline Contract Title and Number: & Period of Performance: \\
\hline & \\
\hline Technologie & Ending Date: \\
\hline
\end{tabular}

Sub-Recipient Project Title:

Modeling of Flotation From First Principles

Principal Investigators:

Roe-Hoan Yoon

Contact Address:

146 Holden Hall

Virginia Polytechnic Institute \& State University

Subcontractor Address:

No subcontracts issued.
Report Information:

Type: Annual

Number: 4

Period: $\quad$ 10/01/03-9/30/04

Date: $\quad 9 / 30 / 04$

Code: VA001-R04

Contact Information:

Phone: (540)231-4508

Fax: (540)231-3948

E-Mail: $\quad$ cast@vt.edu

Subcontractor Information:

Phone:

Fax:

E-Mail:

\section{ABSTRACT}

A turbulent flotation model has been developed by considering the subprocesses of collision, attachment, detachment, and froth recovery. Abrahamson's model (1975) of infinite Stokes number is used to model collision, while the attachment and detachment subprocesses are modeled by considering the kinetic energies required to overcome the energy barriers and the work of adhesion, respectively. The energy barriers are determined by considering various surface forces, including electrostatic, dispersion and hydrophobic forces, while the kinetic energies are determined from the particle velocities at close separation distances from air bubbles. The latter has been determined by modifying the turbulent root-mean-square (RMS) velocities of Liepe and Moeckel (1976) and Schubert (1999) in view of a lubrication theory. In calculating the RMS velocities, it is assumed that a flotation cell is subdivided into low and high energy dissipation zones. The model predictions made by varying various hydrodynamic and surface chemistry parameters are consistent with flotation practice. 


\title{
INTRODUCTION
}

\author{
$\underline{\text { Background }}$
}

Sulman and Packard (1905) was the first to use forced air for flotation. Although the technology has advanced and evolved to many different forms for a wide variety of applications, the basic principles involved in this important solid-solid separation process remain the same. It is a three-phase process, in which one type of particles (solid) is separated from another by air bubbles (gas) in a medium of water (liquid). Hydrophobic particles attach themselves to gas bubbles and rise to the top of a flotation cell and exit the liquid phase while hydrophilic particles remain in the liquid phase. The process is controlled by numerous factors, which may be grouped into hydrodynamic and surface chemical parameters.

The three phases are turbulently mixed to disperse gas phase in liquid phase, to keep solid particles in suspension, and to transport the suspension from one cell to another. The gas bubbles and solid particles collide with each other in the cell, the frequency of collision being determined by the turbulence created by an impeller. The hydrodynamic forces created by the turbulence also affect the efficiency of bubbleparticle adhesion and detachment. Many of the flotation models developed to date use the hydrodynamic parameters affecting the processes of bubble-particle collision, adhesion, and detachment. The model predicting the root-mean-square (RMS) velocity fluctuations of particles and bubbles serve as a basis for studying the effects of various hydrodynamic parameters in flotation (Leipe and Markel, 1976; Schubert, 1999).

Surface forces also play a crucial role in bubble-particle interaction. Derjagiun and Duhkin (1961) were the first to model the process using both hydrodynamic and surface forces. They suggested that during the course of bubble-particle interaction, a particle must pass through three successive zones, i.e., hydrodynamic, diffuso-phoretic and wetting zones. As the particle reaches the wetting zone, it would experience three surface forces, namely, electrostatic, van der Waals dispersion, and structural forces. In a later publication, Derjaguin and Duhkin (1969) suggested that bubble-particle adhesion can occur without penetrating the wetting film, which is referred to as 'contactless flotation', which may explain the difficulty in achieving high-degree of selectivity in fine particle flotation. The structural force was considered 'hydrophilic', which was assumed to be zero when bubble-particle adhesion is to occur.

Pashley and Israelashvili (1982) measured the forces between two mica surfaces that have been hydrophobized with cetylammounium bromide (CTAB). They showed that the structural force, which Derjaguin and Duhkin assumed to be zero, was actually negative (attractive) for hydrophobic surfaces. Follow-up experiments by numerous other investigators also showed the presence of the attractive structural force, which was appropriately referred to as hydrophobic force (Christenson and Claesson, 2001). It was shown that the hydrophobic force increases with increasing water contact angle (Yoon and Ravishankar, 1996). Other investigators showed, on the other hand, that the long- 
range attractions observed with hydrophobic surfaces are actually the capillary forces created by coalescing air bubbles. Ishida et al. (2002) reported indeed that the long-range attractions disappeared when force measurements were conducted in degassed solutions. Mao et al. (2004) used ellipsometry to search for the evidence of nano-bubbles forming on hydrophobic surfaces to no avail. Further, Meyer et al. (2005) measured strong hydrophobic forces between silica surfaces in degassed octadecylammonium chloride (C18TAC) solutions. Similar results have been observed by Zhang, et al. (2005),

Despite the controversy surrounding the existence of hydrophobic force, Yoon and Mao (1996) modeled the subprocess of bubble-particle attachment by comparing the force (or energy) barriers created by surface forces with the kinetic energies available during the process of collision under quiescent conditions. Attachment will be fast if the kinetic energy exceeds the energy barrier. This approach is similar to the concept of fast and slow coagulations depending on the magnitudes of the energy barriers relative to the kinetic energy of the particles involved (Smoluchowski 1917, 1917; Overbeek, 1952; Hunter, 1986). In most cases, both air bubbles and collector-coated particles are negatively charged; therefore, the electrical double layer forces are repulsive. It is well known also that van der Waals dispersion forces between air bubbles and particles are repulsive. Thus, the long-range hydrophobic attraction is the only force that can reduce the energy barriers and, hence, reduce the kinetics of bubble-particle attachment. Indeed, it is well documented in the literature that flotation rate increases with increasing particle hydrophobicity.

The Yoon and Mao's model is based on the first order rate equation:

$$
\frac{d N_{1}}{d t}=-k N_{1}
$$

where $N_{1}$ is the number of particles in a cell, and the rate constant k is given by:

$$
\begin{aligned}
k & =\frac{1}{4} S_{b} P_{c} P_{a}\left(1-P_{d}\right) \\
& =\frac{1}{4} S_{b}\left[\frac{3}{2}+\frac{4 \mathrm{Re}_{b}^{0.72}}{15}\right]\left(\frac{r_{1}}{r_{2}}\right)^{2} \exp \left(-\frac{E_{1}}{E_{k}}\right)\left[1-\exp \left(-\frac{W_{A}+E_{1}}{E_{k}^{\prime}}\right)\right]
\end{aligned}
$$

Here, $S_{b}$ is the surface area rate of air bubbles, $P_{c}$ probability of bubble-particle collision, ${ }^{P_{a}}$ probability of adhesion, ${ }^{P_{d}}$ probability of detachment, Reb bubble Reynolds number, $r_{1}$ particle radius, $r_{2}$ bubble radius, E1 energy barrier for bubble-particle attachment, Ek the kinetic energy for attachment, $E_{k}^{\prime}$ kinetic energy for detachment, and $W_{A}$ is the work of bubble-particle adhesion. Eq. [2] was derived for flotation under quiescent conditions and describes the events occurring in the liquid (or pulp) phase only. Therefore, it may not be applicable for practical applications in industry where turbulence 
is a fact of life and froth phase plays an important role. Nevertheless, the model was based on first principles; therefore, it has diagnostic and predictive capabilities. The model predictions agreed well with the results of the frothless, single-bubble flotation experiments conducted under quiescent conditions.

In the present work, a flotation model has been developed using a similar approach as for developing Eq. [2] but with turbulence and froth phase into consideration, so that it can be of relevance to industrial practice. In this communication, the basic frame work of the model that is currently under development is presented.

\section{$\underline{\text { Objective and Approach }}$}

The objective of this project is to develop a universal model for the froth flotation process. The model will be constructed using the framework originally proposed by Yoon and Mao (1996). In particular, new hydrodynamic expressions will be derived so that the model can be useful for turbulent conditions. Since the modeling expressions will be developed from first principles, the flotation model will have both predictive and diagnostic capabilities. The proposed effort will involve four individual tasks, ie.: Task 1 - Model Development, Task 2 - DPIV Measurements, Task 3 - Model Validation, and Task 4 - Simulator Development.

\section{PROJECT TASKS}

\section{Model Development}

\section{$\underline{\text { Collision Frequency }}$}

Due to the turbulent environment inside a flotation cell, particle-bubble collisions are modeled far differently than in a quiescent environment. For laminar flows, the collision frequency has been modeled by the volume of the slurry swept by a bubble, which can be calculated using the stream functions around the bubble (Mao and Yoon 1997). In a turbulent flow, bubbles and particles do not follow the path of the fluid; therefore, the collision is modeled based upon the deviation of the bubbles and particles from the fluid path. The Stokes number (St), which is a ratio between the particle relaxation (response) time $(\tau i)$ and the smallest fluid relaxation time (Kolmogorov timescale, $\tau \eta$ ), represents how well a particle follows the fluid flow and serves as a means to measure the deviation. If a particle's relaxation time is equal to or less than the Kolmogorov timescale, then the lag time between the fluid movement and the particle movement will be zero and the particle will follow the fluid completely. Any particles with relaxation times above the Kolmogorov timescale will have some departure from the fluid path. Thus, 


$$
S t=\frac{\tau_{i}}{\tau_{\eta}}=\tau_{i} /\left(\frac{v}{\varepsilon}\right)^{1 / 2}
$$

where $v$ is kinematic viscosity of fluid and $\varepsilon$ is energy dissipation. Particle and bubble relaxation times are given by various authors in the literature (Govan 1989; Ceylan, Altunbas et al. 2001; Bourloutski and Sommerfeld 2002).

There are two collision mechanisms involved in turbulent flow field. For particles of Stokes numbers less than 1, collision occurs due to the relative motions of particles (fluid, solid, or gas) in a shear field. For particles of Stokes number of greater than 1, collision occurs due to the acceleration of large and/or heavy particles caused by inertial effects.

Saffman and Turner (1956) proposed a collision model based upon the shear mechanism, where the Stokes number is zero, while Abrahamson (1975) proposed a model based entirely on the accelerative mechanism, where the Stokes number is infinity. There are collision models that combine the shear and accelerative mechanisms (Williams and Crane 1983; Kruis and Kusters 1997), but no model developed to date accounts for the collision between bubbles and particles in water. Modeling collision in water is complicated because bubbles are lighter than the medium, while particles are heavier. In the present work, the Abrahamson's model is used as most particles in flotation have Stokes numbers larger than 1 (Sundaram and Collins 1997).

According to the Abrahamson's model, the collision frequency between particle 1 and bubbles 2 is given by

$$
Z_{12}=2^{3 / 2} \pi^{1 / 2} N_{1} N_{2} d_{12}^{2} \sqrt{\left(\bar{U}_{1}^{2}+\bar{U}_{2}^{2}\right)}
$$

which is a function of the number of particles (N1) and bubbles (N2) per unit volume of slurry, the average collision diameter $(\mathrm{d} 12=\mathrm{r} 1+\mathrm{r} 2)$, and the turbulent root-mean-squared (RMS) velocity fluctuations of particles $\left(\sqrt{\overline{U_{1}}}\right)$ and bubbles $\left(\sqrt{\overline{U_{2}}}\right)$. In the present work, the particle RMS velocities were calculated using the following empirical relationship (Liepe and Moeckel, 1976; Schubert, 1999):

$$
\sqrt{\bar{U}_{1}^{2}}=0.4 \frac{\varepsilon^{4 / 9} d_{1}^{7 / 9}}{v^{1 / 3}}\left(\frac{\rho_{1}-\rho_{3}}{\rho_{3}}\right)^{2 / 3}
$$

Recently, Brady et al. (2004) measured particle velocities using digital particle image velocimetry (PIV) technique and compared the results with those calculated using Eq. [5]. For $80 \mu \mathrm{m}$ particles under grid turbulence, the errors were within $12 \%$. On the other hand, the bubble RMS velocities calculated using the Liepe and Moeckel's model 
were much worse. Therefore, the following equation was used to calculate the bubble RMS velocities (Lee et al., 1987):

$$
\bar{U}_{2}^{2}=C_{0}\left(\varepsilon d_{2}\right)^{2 / 3}
$$

\section{Rate Equation in Pulp Phase}

Knowing the collision frequency (Z12) from Eq. [4] and the corresponding probability of collection $(\mathrm{P})$, it is now possible to calculate the changes in particle concentration (N1) in a flotation cell with time (t), as follows:

$$
\frac{d N_{1}}{d t}=-Z_{12} P_{A}\left(1-P_{D}\right) R_{F}
$$

where PA is the probability of attachment, PD the probability of detachment, and RF is the fractional recovery in froth phase.

Comparing Eq. [7] with Eq. [1], one obtains the following:

$$
k=\frac{Z_{12} P_{A}\left(1-P_{D}\right) R_{F}}{N_{1}}
$$

as an expression for the first-order rate constant for the flotation processes occurring under turbulent conditions. Let $Z_{12}=\Omega N_{1} N_{2}$, then Eq. [8] is reduced to:

$$
k=\Omega N_{2} P_{A}\left(1-P_{D}\right) R_{F}
$$

In the model developed by Yoon and Mao (1996), the probability of attachment, PA, was considered to be a function of the energy barrier (E1) for bubbleparticle attachment and the kinetic energy of attachment (Ek),

$$
P_{A}=\exp \left(-\frac{E_{1}}{E_{k}}\right)
$$


while PD is a function of work of adhesion (WA), E1 and the kinetic energy of detachment (Ek').

$$
P_{D}=\exp \left(-\frac{W_{A}+E_{1}}{E_{k}^{\prime}}\right)
$$

Yoon and Mao (1996) showed that

$$
W_{A}=\gamma_{l v} \pi r_{1}^{2}(1-\cos \theta)^{2}
$$

where $\gamma \mathrm{lv}$ is the surface tension of water and $\theta$ is the water contact angle of the particle with radius $\mathrm{r} 1$.

In the present work, the energy barrier E1 has been determined using the extended DLVO theory,

$$
V_{T}=V_{E}+V_{D}+V_{H}
$$

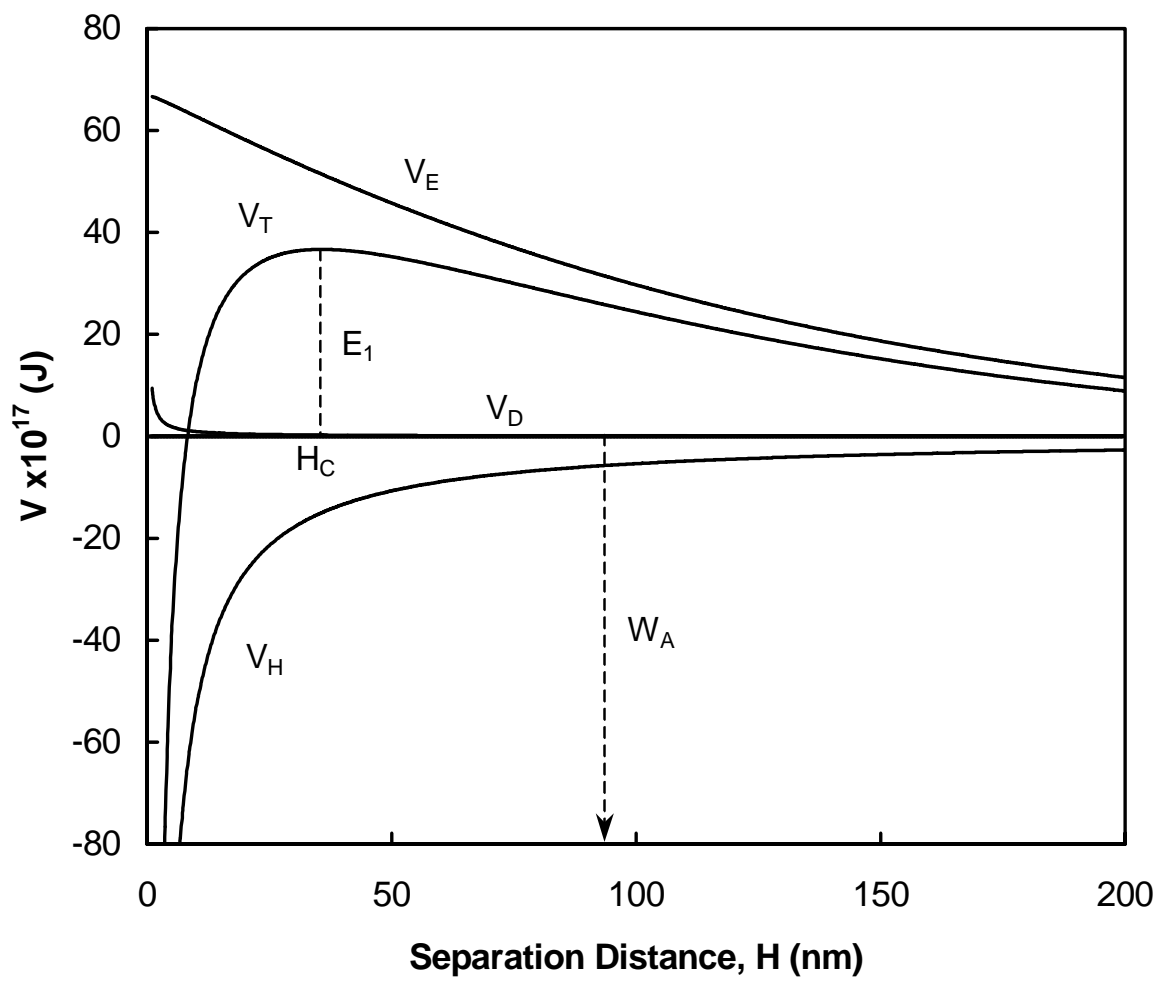

Figure 1. Surface energy vs. distance diagram for bubble-particle interactions: $\mathrm{V}_{\mathrm{E}}=$ electrostatic repulsive energy; $V_{D}=$ dispersion interaction energy; $V_{H}=$ hydrophobic interaction energy; $V_{T}=V_{E}+V_{D}+V_{H} ; E_{1}=$ energy barrier; $H_{c}=$ critical rupture thickness; $\mathrm{W}_{\mathrm{A}}=$ work of adhesion. 
which states that the free energy of interaction, VT, between two particles is given as the sum of electrostatic, VE, van der Waals dispersion, VD, and hydrophobic, VH, interaction energies. Under most flotation conditions particularly for flotation in alkaline conditions, both VE and VD are repulsive, while VH is attractive as shown in Figure 1.

The electrostatic interaction energy, VE, can be calculated using the following relation (Hogg, Healey and Fuerstenau, 1966)

$$
V_{E}=\frac{\pi \varepsilon_{0} \varepsilon r_{1} r_{2}\left(\Psi_{1}^{2}+\Psi_{2}^{2}\right)}{\left(r_{1}+r_{2}\right)}\left[\frac{2 \Psi_{1} \Psi_{2}}{\Psi_{1}^{2}+\Psi_{2}^{2}} \ln \left(\frac{1+e^{-\kappa H}}{1-e^{-\kappa H}}\right)+\ln \left(1-e^{-2 \kappa H}\right)\right]
$$

where 0 is permittivity in vacuum, . is the dielectric constant of the medium, $r 1$ and $r 2$ are the particle and bubble radii, respectively, 1 and 2 are the double layer potentials for particle and bubble, respectively, is the inverse Debye length, and $\mathrm{H}$ is the separation distance between particle and bubble. As an approximation, 1 and 2 may be substituted by potentials.

The dispersion interaction energy can be calculated using the following relation (Rabinovich and Churaev, 1979),

$$
V_{D}=-\frac{A_{132} r_{1} r_{2}}{6 H\left(r_{1}+r_{2}\right)}\left[1-\frac{1+2 b l}{1+b c / H}\right]
$$

where A132 is the Hamaker constant for the interaction between particle 1 and bubble 2 in a medium 3. The second term of Eq. [15] is a correction factor for the retardation effect, where $b(=3 \times 10-17 \mathrm{~s}$ for most materials $)$ is a parameter characterizing materials of interacting particles, $1(=3.3 \times 1015 \mathrm{~s}-1$ for water $)$ is a parameter characterizing the medium, and $\mathrm{c}$ is the speed of light (Yoon and Mao, 1997).

The hydrophobic interaction energy $(\mathrm{VH})$ between a bubble and a particle may be calculated using the following equation (Yoon and Mao, 1996).

$$
V_{H}=-\frac{r_{1} r_{2} K_{132}}{6\left(r_{1}+r_{2}\right) H}
$$

which is of the same form as the dispersion interaction represented by Eq. [15]. The hydrophobic interaction constant, K132, can be obtained using the combining law (Yoon, Flinn, and Rabinovich, 1997):

$$
K_{132}=\sqrt{K_{131} K_{232}}
$$

in which K131 and K232 represent the hydrophobic force constant between two particles 1 and the same between two air bubbles 2, respectively. 
Many investigators showed that the long-range attractive force between two hydrophobic surfaces increases with increasing hydrophobicity. Pazhianur and Yoon (2003) showed that K131 increases with increasing water contact angle $(\theta)$, a measure of hydrophobicity. The $\log \mathrm{K} 131$ vs. $\theta$ plots given by these investigators may be used to obtain the values of the hydrophobic force constants from the contact angles that can be more readily measured in experiment. The plot shows three different regions of contact angles, in which one can obtain the value of K131 from $\theta$ using the following relation:

$$
K_{131}=a \exp \left(b_{k} \theta\right)
$$

in which a and bk are fitting parameters whose values are: $\mathrm{a}=2.732 \times 10-21$ and $\mathrm{bk}=$ 0.04136 at.$<86.89^{\circ} ; \mathrm{a}=4.888 \times 10-44, \mathrm{bk}=0.6441$ at $86.89^{\circ}<.<92.28^{\circ}$; and $\mathrm{a}=$ $6.327 \times 10-27$, bk $=0.2172$ at . $>92.28^{\circ}$.

Yoon and Aksoy (1999) and Wang and Yoon (2004) showed that the air bubble produced in pure water is hydrophobic and its hydrophobic force constant K232 decreases with increasing surfactant concentration. The following empirical relationship,

$$
K_{232}=\exp (d+e \sqrt{C})
$$

may be used to obtain the value of K232 from the molar concentration, C, of an ionic surfactant. The data obtained with dodecylammonium hydrochloride as a surfactant (Yoon and Aksoy, 1999) can be fitted with the two fitting parameters, i.e., $d=-39.67$ and $\mathrm{e}=-117.7$.

To calculate PA using Eq. [10], it is necessary to know the kinetic energy, Ek, of bubble-particle collision. There are two sources of energies for the collision. One is created by gravity and the other by turbulent agitation. The former causes particles to settle and bubbles to rise. In the present work, the velocities of both settling particles and rising air bubbles have been calculated using the equations of Dobby and Finch (1990), which were derived on the basis of the work of Masliyah (1979). It is considered that setting and rising velocities are directed toward each other along the vertical direction. The turbulent agitation, on the other hand, creases a spectrum of turbulent vortices of different dimensions, ranging from macroscale, $\Lambda$, which is of the order of impeller, to Kolmogorov microscale, $\lambda \mathrm{D}$. The latter is defined as

$$
\lambda_{D}=\left(\frac{v^{3}}{\varepsilon}\right)^{1 / 4}
$$

where $\varepsilon$ is the rate of energy dissipation. In general, the bubbles and particles in flotation interact with vortices of larger than this dimension. In the present work, Eqs. [5] and [6] have been used to calculate the turbulent relative velocities of particles and bubbles, 
respectively. It is considered that the turbulent velocities, i.e., $\sqrt{\bar{U}_{1}^{2}}$ and $\sqrt{\bar{U}_{2}^{2}}$, are directed toward each other.

It is assumed that the rising/settling velocities are in the vertical plane (due to gravity), while the turbulent velocities are in the horizontal plane (due to impeller). The net relative velocity, $U_{12 N e t}$, at which a bubble and a particle collide with each other is then given by

$$
U_{12 \text { Net }}^{2}=U_{12 T u r}^{2}+U_{12 R / S}^{2}
$$

where $U_{12 T u r}$ is the turbulent collision velocity due to turbulence and $U_{12 R / S}$ is the same due to gravity. One can then calculate Ek using the following equation:

$$
E_{k}=\frac{1}{2}\left(m_{1}+m_{2}\right) U_{12 N e t}^{2}
$$

where $\mathrm{m} 1$ and $\mathrm{m} 2$ are the masses of particle and bubble, respectively.

In calculating the velocities of particles and bubbles using Eqs. [5] and [6], there are two choices regarding the value of energy dissipation rate. One is to use a mean value $(\varepsilon)$ for the entire flotation cell and the other is to use local dissipation rate $(\varepsilon d)$. The latter has been chosen in the present work. Thus,

$$
\varepsilon_{d}=\varphi \frac{P}{M}
$$

where $\mathrm{P}$ is power input, $\mathrm{M}$ mass of the slurry, and $\varphi$ is the local energy dissipation coefficient, which is the ratio of $\varepsilon d$ to $\varepsilon$. In engineering systems, the peak value of $\varphi$ is 5 to 200 times the mean value (Schultze, 1984). Lu et al. (1998) reported the value of $\varphi=$ 50 at the tip of an impeller of a laboratory mixer. In the present work, it is assumed that $\varphi$ $=0.01$ for the calculation of Ek for attachment. Eq. [22] may also be used for the calculation of Ek' for detachment, for which it is assumed that $\varphi=200$. These assumptions suggest that bubble-particle attachment can occur at relatively low kinetic energies, while detachment requires higher kinetic energies which are imparted near the impeller. Detachment can occur in relatively large vortices, in which bubbles move towards the center and particles toward the outside due to their different relative densities in a liquid phase (Crowe et al., 1995; Chahine, 1995).

As a particle approaches a bubble, the distance, $\mathrm{H}$, between the two decreases with time. As $\mathrm{H}$ reaches the critical rupture distance $\mathrm{Hc}$, the wetting film ruptures spontaneously and a three-phase contact is formed. It is necessary, therefore, to compare Ek with the energy barrier E1 at Hc, which in turn requires the velocity of the particle at the rupture thickness rather than in the bulk of the solution. According to the Reynolds 
lubrication theory, which was developed for a sphere approaching a flat surface, the particle should experience a hydrodynamic resistance force Fhyr during the process of film thinning:

$$
F_{\text {hyd }}=6 \pi \mu r_{1} U \beta
$$

where $\mathrm{U}$ is the velocity of the sphere at $\mathrm{H}, \mu$ is the viscosity, and is a dimensionless parameter, which is given as the ratio between $\mathrm{r} 1$ and H. In the present work, the values of have been determined using the following relationship (Barnocky and Davis, 1989),

$$
\beta=\frac{r_{1}}{4 H}
$$

which is derived for a small sphere approaching a large sphere. By equating the lubrication resistance force at $\mathrm{Hc}$ to the drag force on a sphere of radius $\mathrm{r}$, one obtains the following (Luttrell \& Yoon, 1992):

$$
U_{H_{c}}=\frac{U}{\beta}
$$

which can be used to determine the velocity of the particles colliding with bubbles at Hc. It is this velocity rather than the velocity in the bulk solution that is used to calculate Ek.

\section{Froth Recovery}

Froth behavior is very complex. The loss of bubble surface area due to coalescence, and the drainage of liquid from lamella and plateau boarders affect the fate of particles in a froth phase. Also, particles can either stabilize or destabilize froth depending on the hydrophobicity and the size of the particles present in a froth phase. For various reasons, only a fraction, $\mathrm{Rf}$, of the particles entering a froth is recovered, while the rest is returned to the pulp. A simple approach to modeling the recovery process in a froth phase would be to assume that $\mathrm{Rf}$ is proportional to the bubble surface area. Thus,

$$
R_{F}=\frac{S_{f}}{S_{0}}=\left(\frac{3 V_{g}}{r_{2-f}}\right) /\left(\frac{3 V_{g}}{r_{2-0}}\right)=\frac{r_{2-0}}{r_{2-f}}
$$

where S0 and Sf represent the surface area rates at the bottom and top of a froth, respectively. As shown, froth recovery is equal to the bubble size ratio at the top and bottom of a froth, which would be the case for flotation operating under the maximum carrying capacity limit.

Gorain et al. (1998) proposed a simple froth recovery model: 


$$
R_{F}=\exp \left(-\alpha \tau_{f}\right)
$$

which suggests that the recovery varies with the retention time, f, of air in a froth phase, which is obtained by dividing froth height with superficial gas rate Vg. . is an adjustable parameter, which varies in the range of 0.1 and 0.5 .

\section{RESULTS AND DISCUSSION}

Simulations were run to show the effect of different variables on the flotation rate constant. The variables studied included the particle diameter (Dp), bubble diameter $(\mathrm{Db})$, surface tension $(\gamma \mathrm{lv})$, contact angle $(\theta)$, zeta potentials of particles $(\zeta \mathrm{p})$ and of bubbles $(\zeta \mathrm{b})$, and specific energy input ( $\varepsilon \mathrm{sp})$. All of the simulation results presented in this communication were obtained using local energy dissipation $(\varepsilon d)$ rather than the mean energy dissipation $(\varepsilon)$. As has already been note, it was assumed that the local energy dissipation coefficient $(\varphi)$ was 0.01 for attachment and that it was 200 for detachment. These values are not far off the local energy dissipation rates measured near the impeller (Lu, et al. 1998; Pyke, et al., 2002).

Unless otherwise stated, the values of the different parameters used for simulations were as follows: $\varepsilon \mathrm{sp}=2 \mathrm{~kW} / \mathrm{m} 3, \mathrm{Db}=1 \mathrm{~mm}, \zeta \mathrm{p}=-20 \mathrm{mV}, \zeta \mathrm{b}=-30 \mathrm{mV}, \gamma \mathrm{lv}$ $=68.0 \mathrm{mN} / \mathrm{m}$. The density of the particle was $2,650 \mathrm{~kg} / \mathrm{m} 3$, which was for silica. It was assumed that the contact angle $\theta$ of the silica particles was $60 \mathrm{o}$, which gave the value of $\mathrm{K} 131=3.27 \times 10-20 \mathrm{~J}$ using the appropriate fitting parameters of Eq. [18].

The Hamaker constants used in the present work were $5.04 \times 10-20 \mathrm{~J}$ for silica, 0 for air, and 4.38x10-20 J for water, from which the value of A132 = -3.18x10-21 J was obtained using the geometric mean combining law. Substituting this value to Eq. [15], it was possible to calculate the repulsive dispersion interaction energy (VD). Since A132 was negative, VD is repulsive for bubble-particle interactions. The electrostatic interaction energy (VE) was calculated with Eq. [14] using $\zeta \mathrm{p}$ and $\zeta \mathrm{b}$ for 1 and 2 as approximation and using $\kappa-1=96 \mathrm{~nm}$ as the Debye length. The hydrophobic interaction energy (VH) was calculated using Eq. [16] with the value of $\mathrm{K} 132=4.14 \times 10-19 \mathrm{~J}$, which in turn was obtained using Eq. [17] from the values of K131 = 3.27x10-20 J and K232 = 5.25x10-18 J. Regarding physical variables, the superficial air flow rate was $1.0 \mathrm{~m} / \mathrm{s}$, and the froth height was kept constant at $0.4 \mathrm{~m}$. The adjustable parameter of the froth recovery model (Eq. [25]) was assumed to be 0.03 . 
Figures 2 and 3 show the effects of physical variables, i.e., bubble size (Db) and energy dissipation ( $\varepsilon \mathrm{sp})$ on flotation rate constant $(\mathrm{k})$. The simulation results obtained by varying $\mathrm{Db}$ and particle size $(\mathrm{Dp})$ are plotted versus the latter in Figure 2. Before discussing the effects of changing $\mathrm{Db}$ and $\mathrm{Dp}$, it is noted here that the magnitudes of $\mathrm{k}$ values obtained from the simulation are in general agreements with the experimental values reported in the literature. Ahmet and Jameson (1985) reported $\mathrm{k}$ in the range of 0.01 to $3 \mathrm{~min}-1$ for the flotation of quartz, depending on the particle size and agitation speed. For the flotation of sulfide minerals, the $\mathrm{k}$ values were in the range of 0.2 to 5 min1 (Trahar, 1981). It is also important to note that $\mathrm{k}$ versus Dp plots are symmetric, that is, there exists an optimum particle size for flotation at a given bubble size. The recovery versus particle size plots reported by Trahar and Warren (1976) are also symmetric, showing that flotation is effective over a relatively narrow particle size ranges. A plant survey reported by Gaudin et al. (1931) show that the flotation recovery of various sulfide minerals drops sharply above $100 \mu \mathrm{m}$ but gradually below $10 \mu \mathrm{m}$. De Bruyn and Modi (1956) showed in laboratory-scale continuous flotation tests that the optimum particle size range for silica was 10 to $40 \mu \mathrm{m}$. These results show that flotation is difficult with fine and coarse particles. The difficulty in floating fine particles is attributed to the

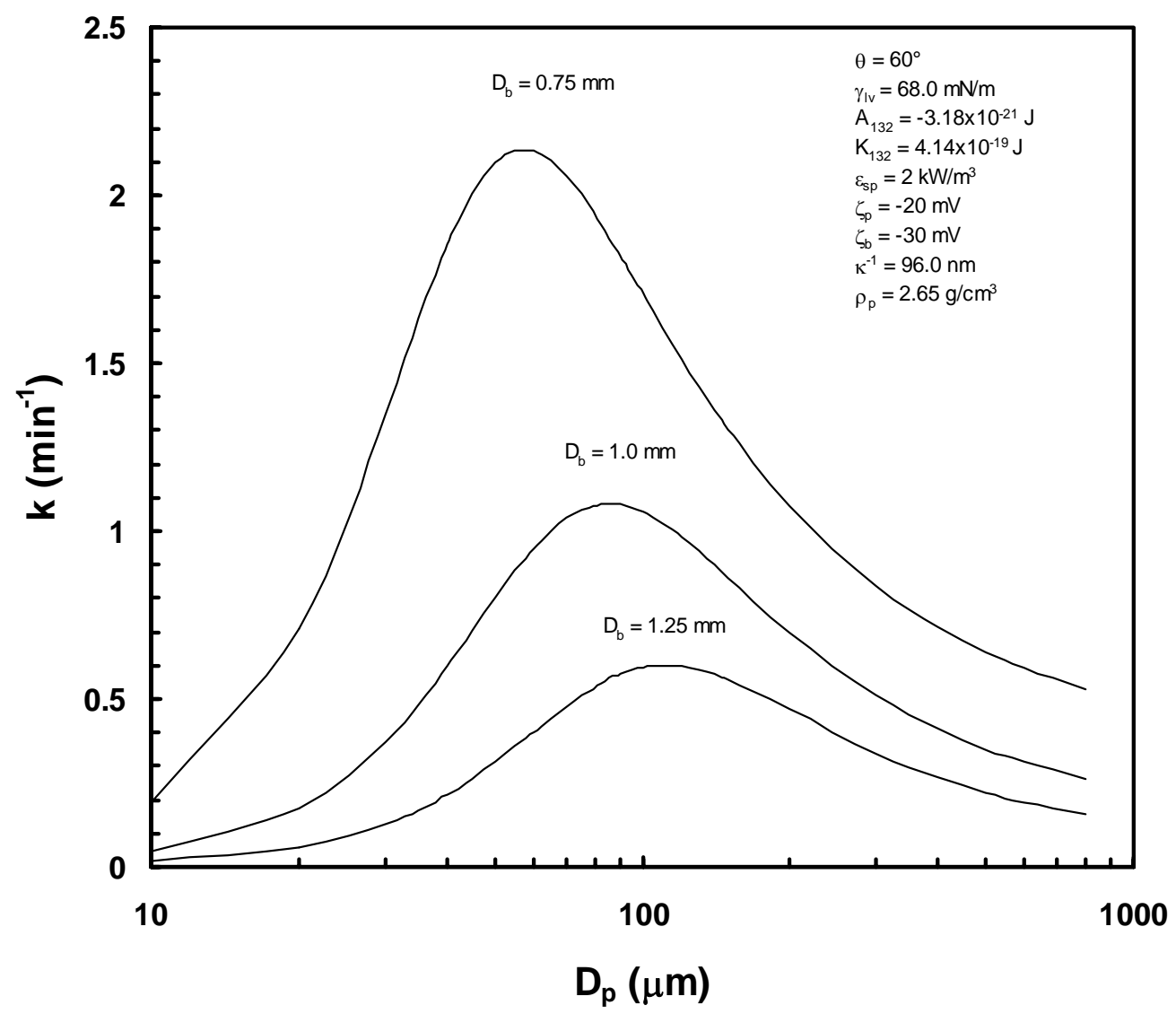

Figure 2. Effect of bubble size $\left(\mathrm{D}_{\mathrm{b}}\right)$ on flotation rate constant $(k)$. Smaller bubbles give higher rates at a given particle size $\left(D_{p}\right)$, and the optimum particle size for flotation shifts to smaller sizes, indicating that smaller bubbles are beneficial for fine particle flotation. 
low collision frequency (Z12). As shown in Eq. [4], Z12 decreases with decreasing Dp $(=2 \mathrm{r} 1)$. The difficulty in floating coarse particles, on the other hand, is due to the high probability of detachment (PD). As the particle size is increased, the turbulent RMS velocity $\left(\sqrt{\bar{U}_{1}^{2}}\right)$ is increased as shown by Eq. [5], which should in turn cause the kinetic energy of detachment (Ek') to increase. The simulation results given in Figure 2 show also that the use of smaller air bubbles shifts the optimum particle size at which maximum $\mathrm{k}$ is obtained toward a lower particle size. This finding, which can be attributed to the lower probability of detachment associated with using smaller air bubbles, suggests that the difficulty in floating fine particles can be minimized by using smaller air bubbles. Many investigators suggested the same (Ahmet and Jameson, 1985; Yoon and Luttrell, 1989).

The outcome from varying the specific energy input $\varepsilon s p$, along with the particle size Dp, is shown in Figure 3. It shows that as the energy input is increased, the rate constant $\mathrm{k}$ is shown to decrease. This may seem counter-intuitive and opposite of what is observed in flotation practice. Ahmet and Jameson (1985) showed, however, that an increase in agitation caused an increase in the rate of flotation of latex particles. For particles of higher specific gravity, agitation caused a decrease in flotation rate when using smaller air bubbles. A problem in comparing the results of the simulation with actual flotation is that the present simulation was run at a $\mathrm{Db}$ of $1 \mathrm{~mm}$, while bubble size can change with $\varepsilon s p$ in actual flotation. In general, bubble size decreases with increasing esp (Schulze, 1984; Schubert, 1999; Brake et al, 1996). The results presented in Figure 3 are an indication that at a given bubble size, an increase in energy input causes an

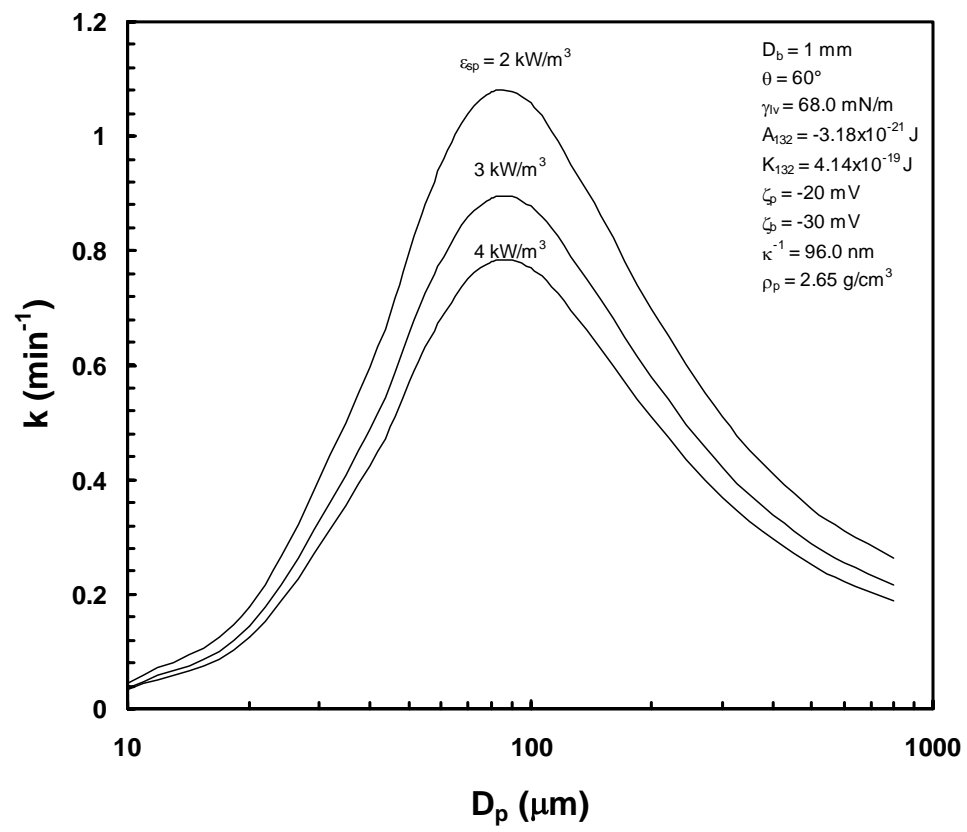

Figure 3. Effect of specific energy input $\left(\varepsilon_{\mathrm{sp}}\right)$ on flotation rate constant $(k)$ at $1 \mathrm{~mm}$ bubble size $\left(D_{b}\right)$. A higher energy input causes an increase in the kinetic energy $\left(E_{k}{ }^{\prime}\right)$ of detachment and hence a decrease in $\boldsymbol{k}$. 
increase in the kinetic energy of detachment (Ek') and, hence, a decrease in flotation rate.

Figures 4 to 6 show the effects of various surface chemistry parameters. The results of varying the contact angle $(\theta)$, along with particle size $(\mathrm{Dp})$, have been plotted versus the latter in Figure 4. As shown, the rate constant $\mathrm{k}$ increases with increasing particle hydrophobicity. An increase in hydrophobicity should have triple benefits. First, an increase in hydrophobicity should increase the work of adhesion (Wa) and, hence, decrease the probability of detachment (PD) as suggested by Eqs. [11] and [12]. Second, an increase in hydrophobicity should increase the hydrophobic force constant K131, which should in turn reduce the energy barrier E1 and, hence, PA as suggested by Eq. [10]. Third, an increase in hydrophobicity should increase the critical rupture thickness $(\mathrm{Hc})$, which should increase the kinetic energy of attachment (Ek) and, hence, the probability of attachment (PA) as suggested by Eq. [10].

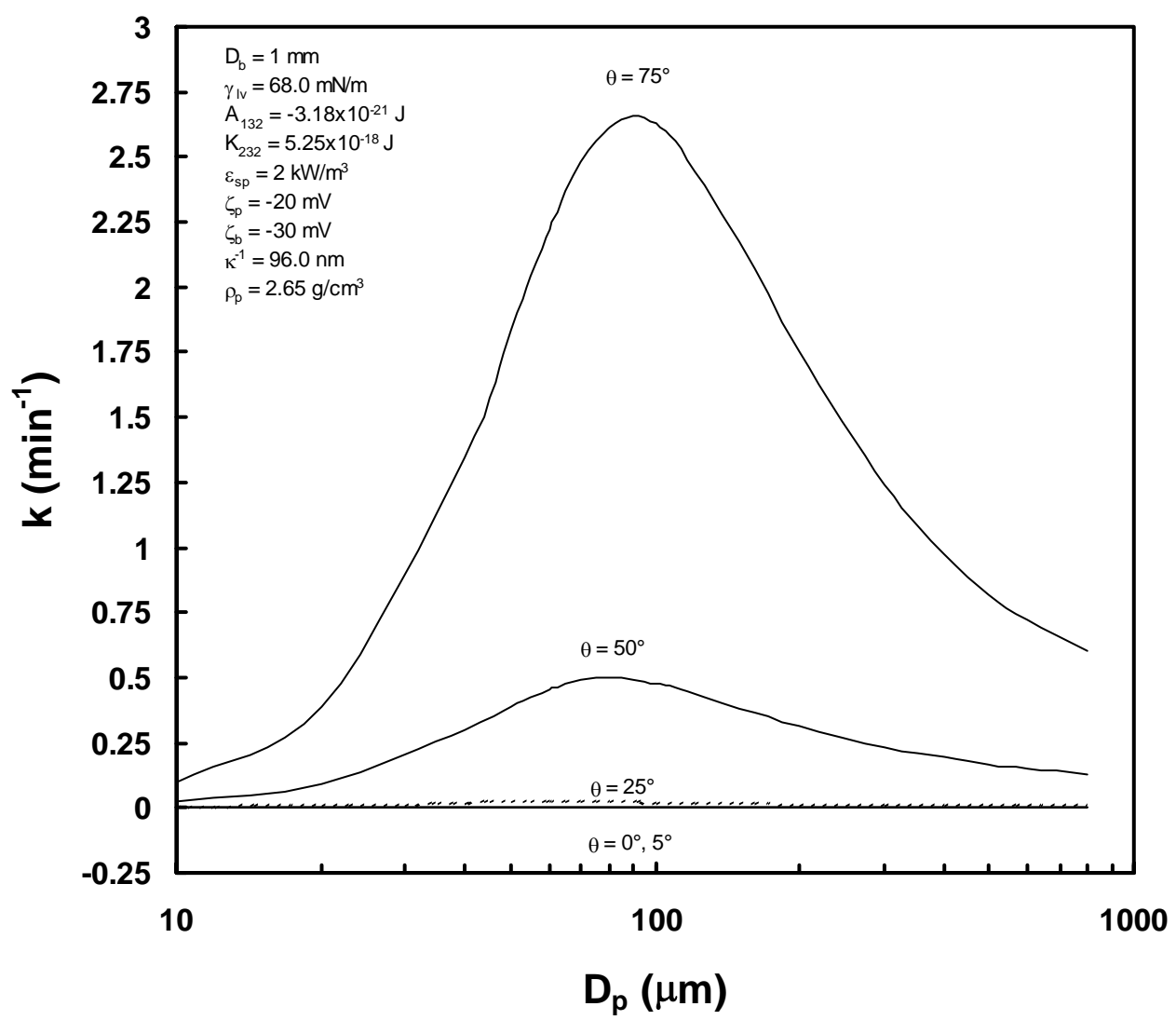

Figure 4. Effect of contact angle $(\theta)$ on flotation rate constant $(k)$. An increase in contact angle causes the energy barrier $\left(E_{1}\right)$ to decrease and the work of adhesion $\left(W_{A}\right)$ to increase, both of which contribute to increase the rate constant. 


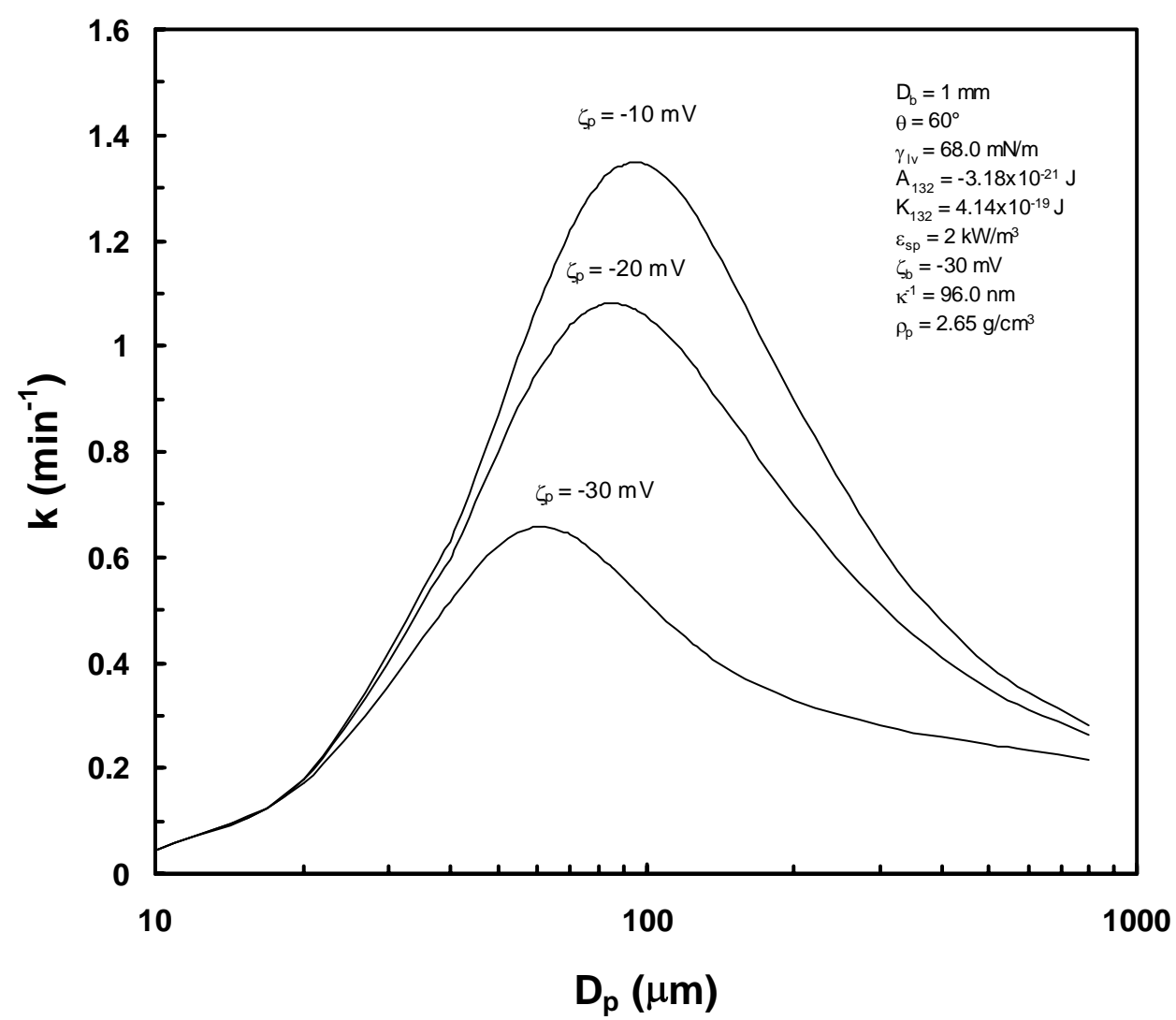

Figure 5. Effect of particle zeta potential $\left(\zeta_{p}\right)$ on flotation rate constant $(k)$ at the bubble zetapotential $\left(\zeta_{\mathrm{b}}\right)$ of $-\mathbf{3 0} \mathrm{mV}$. $k$ increases with a decrease in the negative zeta-potential of the particles due to a decrease in energy barrier $\left(E_{1}\right)$.

Figure 5 shows the simulation results obtained by varying the zeta-potentials $(\zeta \mathrm{p})$ of particles at different particle sizes. It shows that the rate constant $(\mathrm{k})$ increases substantially as $\zeta \mathrm{p}$ becomes less negative. Since the zeta-potential of air bubbles was negative $(\zeta \mathrm{b}=-30 \mathrm{mV})$, a decrease in the negative zeta-potential should result in a decrease the energy barrier (E1) and, hence, an increase in k. This result is in agreement with the work of many investigators, who showed that flotation recovery is maximum when the magnitude of zeta-potential is minimum (Fuerstenau, 1957; Jaycock and Ottewill, 1963; Baker and Miller, 1968; DeVivo and Krager, 1970; Chander and Fuerstenau, 1972; Ray and Ratcliff, 1973). The role of zeta-potential (or surface potential) has long been known. In the early 1930's, Russian researchers discovered that coal and other hydrophobic minerals such as sulfur, talc, and graphite could be floated in solutions of inorganic electrolytes without the use of either collector or frother. The major improvements were found in the kinetics of flotation (Klassen and Mokrousov, 1963; Glevov and Konishin, 1974). Several coal cleaning plants took advantage of this phenomenon by using waste solutions from soda plants, containing $\mathrm{CaCl} 2 \mathrm{~m} \mathrm{NaCl}$, and 
$\mathrm{CaCl} 2$, along with their regular flotation reagents, and achieved improved performance (Glembotskii, et al., 1963). The process of using inorganic electrolytes in flotation is known as "salt flotation". Laskowski (1965) suggested that electrolytes compress the electrical double-layer and reduce the disjoining pressure of the wetting film, which is conducive to bubble particle attachment.

There may be a close analogy between flotation and coagulation. If there is no energy barrier, i.e., $\mathrm{E} 1 \leq 0$, the coagulation is referred to as 'fast' (Smoluchowski 1917, 1917; Overbeek, 1952). If E1 $\geq 0$, coagulation is called 'slow'; however, it can give its way to fast coagulation by bringing the $\mathrm{pH}$ to the iso-electric point (i.e.p.) of the colloidal particles or by adding an electrolyte to compress the electrical double layer (Hunter, 1986).

It may be of interest to note here that a decrease in the negative zeta-potential causes the critical rupture thickness $(\mathrm{Hc})$ to increase, which in turn increases the kinetic energy of attachment (Ek). At a larger Hc, the velocity of the particle approaching an air bubble becomes higher, because the hydrodynamic resistance to film thinning becomes smaller at a larger Hc (see Eqs. [21]-[23]). Thus, the decrease in negative zeta-potential have dual benefits, that is, E1 to decrease and Ek to increase, both of which should contribute to the increase in $\mathrm{k}$.

Figure 5 shows also that the optimum particle size at which $\mathrm{k}$ reaches a maximum shifts toward a smaller particle size as the negative zeta-potential is decreased. This observation can be explained by the increase in Ek due to the increase in Hc, which should help smaller particles, more than the larger ones, to overcome the energy barrier.

The final surface force parameter that was studied was the surface tension $(\gamma \mathrm{lv})$. The results of the simulation are plotted as a function of particle size in Figure 6. It is shown that flotation rate constant decreases with decreasing surface tension, which is entirely due to the changes in work of adhesion (Wa) (see Eq. [12]). This finding suggests that excessive use of frother may be detrimental to coarse particle flotation. It should be noted, however, that the present simulation did not consider the effect of $\gamma \mathrm{lv}$ on bubble size.

The model developed in the present work is by no means complete, as flotation is a complex three-phase phenomenon and there are many unknowns. The most difficult part of modeling flotation under turbulent flow conditions is to accurately determine the kinetic energies involved in the subprocesses of bubble-particle attachment and detachment. In the present work, a flotation cell was subdivided into two compartments, i.e., high and low energy dissipation zones. It may be necessary, however, to use a multi-compartment model for energy dissipation. In the present work, a relatively simple froth recovery model was used. A preliminary work conducted using a froth model incorporating particle size effect allows the local energy dissipation coefficient $(\varphi)$ to be substantially less than what has been used in the present work. 


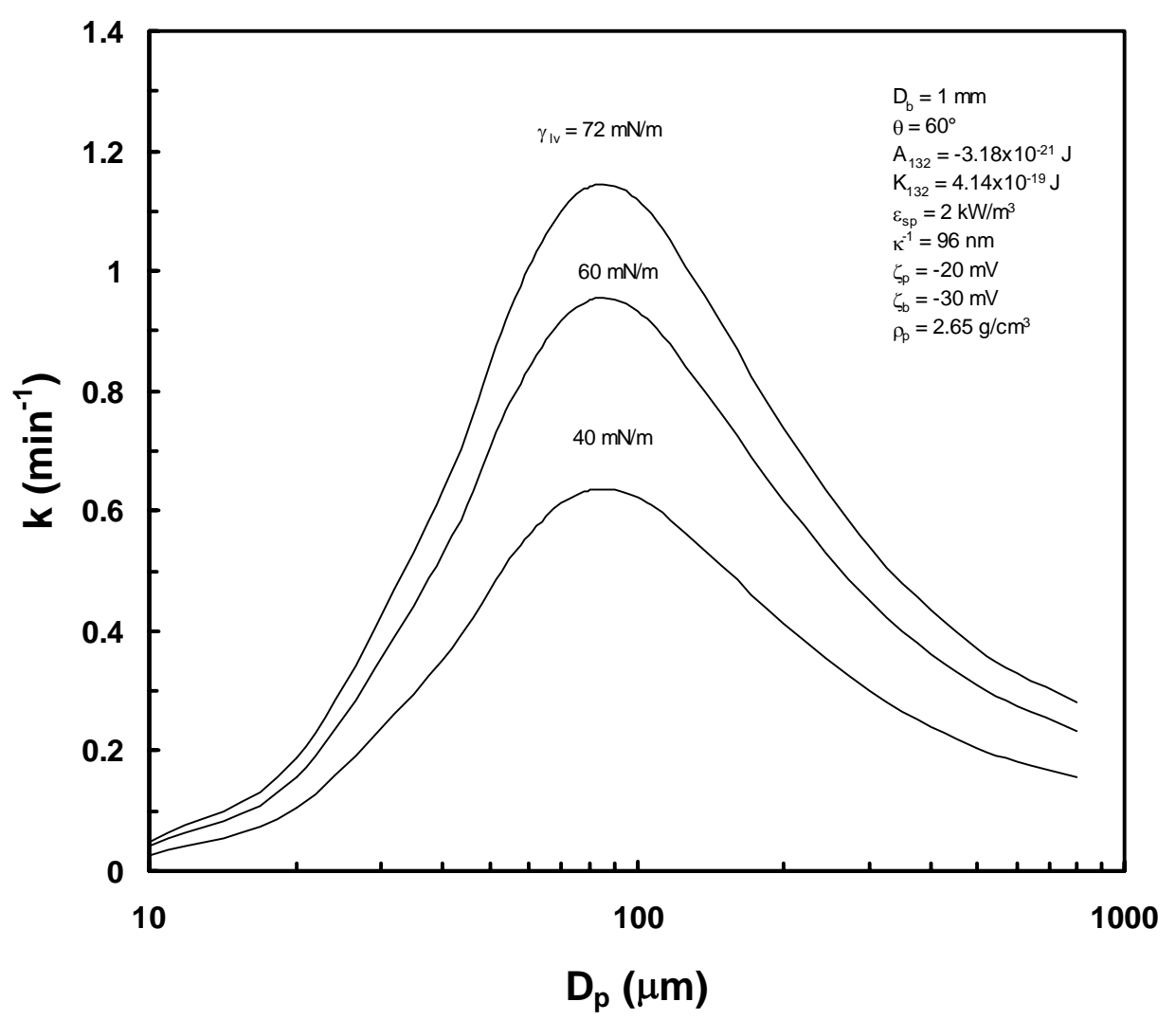

Figure 6. Effect of surface tension $\left(\gamma_{1 v}\right)$ on flotation rate constant $(k)$. A lower surface tension causes a decrease in work of adhesion $\left(\mathrm{W}_{\mathrm{A}}\right)$ and hence a decrease the probability of detachment $\left(\mathrm{P}_{\mathrm{A}}\right)$.

\section{SUMMARY}

A flotation model was developed that can predict flotation from both hydrodynamic and surface chemistry parameters under turbulent flow conditions. It was developed by considering the subprocesses of collision, attachment, detachment, and froth phase recovery. The collision subprocess was based on Abrahamson's model, which assumes a Stokes number of infinity. The attachment subprocess was modeled by considering the energy barriers relative to the kinetic energies involved. The energy barriers were determined by considering the surface forces, while the kinetic energies were calculated using the particle velocities at the critical rupture thickness of the wetting film. The detachment subprocess was modeled from the work of adhesion and kinetic energies of detachment. The kinetic energies were calculated by assuming a twocompartment energy dissipation model. The froth recoveries were calculated using the Gorain et al.'s model.

The model was used to simulate flotation using both hydrodynamic and surface chemistry parameters under turbulent conditions. The simulation results show that flotation is efficient at a relatively narrow range of particle sizes. It is also shown that 
floatation rate is critically dependent on contact angle, zeta-potential and surface tension, as has been amply demonstrated in the literature and in flotation practice.

\section{FUTURE WORK}

Further refinement of the flotation model will continue during the next reporting period. This will include a more in-depth theoretical analysis of the effects of turbulence on particle and bubble movements. Experimental work will include the flotation tests to validate the model as well as DPIV data analysis. Also, the froth model presented here will be modified so that it considers the effect of particle size.

\section{REFERENCES}

Abrahamson, J., Chemical Engineering Science, 30, 1371 (1975).

Ahmed, N. and Jameson, G.J., Int. J. Miner. Process., 14, 195 (1985).

Baker, H. F. and Miller, K.J., Mining Congress Journal, 55, 43-44, (1968).

Barnocky, G. and Davis, R. H., Int. J. Multiphase Flow, 15(4), 627-638 (1989).

Bourloutski, E. and Sommerfeld, M., FED (American Society of Mechanical Engineers) 257-2 (A, Proceedings of the 2002 ASME Joint U.S.-European Fluids Engineering Conference), 355-364 (2002).

Brady, M.R., Vlachos, P.P. and Telionis, D.P., Sherrell, I. and Yoon, R.-H., SME

Annual Meeting, February 23-25, 2004, Denver, CO., Preprint 04-193.

Chander, S. and Fuerstenau, D. W., Trans AIME, 252, 62 (1972).

Ceylan, K., A. Altunbas, et al., Powder Technology, 119, 250 (2001).

Chahine, G. L., Fluid Vortices, ed. by Green, S. I., 783 (1995).

Christenson, H.K. and Claesson, P.M., Adv. Colloid and Interface Science, 91, 391 (2001).

Crowe, C. T., Trout, T. R. and Chung, J. N., Fluid Vortices, ed. by Green, S. I., 829 (1995).

De Bruyn, P.L. and Modi, H.J., Mining Eng., 8, 415 (1956).

Derjaquin, B.V. and Dukhin, S.S., Transactions, Institute of Mining and Metallurgy 70, 221 (1961).

Derjaguin, B. V. and Duhkin, S. S., Trans. Inst. Min. Metall. 70, 221 (1961).

Derjaguin, B. V. and Duhkin, S. S., Proceedings 13 Int. Mineral Processing Congr. 2, 21 (1969).

DeVivo, D.G. and Krager, B.L., Separation Science, 5, 145 (1970).

Dobby, G.S. and Finch, J. A. in Column Flotation. Pergamon (1990).

Fuerstenau, D.W., Trans. Am. Inst. Mining Eng., 208, 1365 (1957).

Gaudin, A.M., Groh, J.O. and Henderson, H.B., AIME Eng., Tech. Pub. No. 414 (1931).

Glembotski, V.A. in Flotation, pp. 396-414, Translated by W.W. Hammond, New York, Prime Sources (1963).

Glevov, Yu. M. and Konishin, V.V. Obogashchenie Rud 4 (12), 9 (1974).

Gorain, B. K., Harris, M. C., Franzidis, J.-P. and Manlapig, E. V., Minerals Engineering 11(7), 627 (1998). 
Govan, A. H., International Journal of Multiphase Flow, 15(2), 287 (1989).

Hogg, Healey and Fuerstenau, 1966

Hunter, R.J., in Fundamentals of Colloid Science, Oxford Science Publications, pp. 94-97 (1986)

Ishida, N., Sakamoto, M., Miyahara, and Higashitani, K., Lanmuir, 16, 5681 (2000).

Jacock, M.J. and Ottewill, R.H., Trans IMM, 72, 497 (1963).

Klassen,V.I and Mokrousov, V.A. in An introduction to the Theory of Flotation, pp. 33842. London:Butterworths \& Co., Ltd. (1963).

Kruis, F. E. and Kusters, K. A. Chemical Engineering Communications, 158, 201 (1997).

Laskowski, J., Colliery Guardian 211, 361 (1965).

Lee, C. H., Erickson, L. E., et al. Chemical Engineering Communications, 59 (1-6), 65 (1987).

Liepe, F. and Moeckel, H. O., Chemische Technik 28 (4), 205 (1976).

Lu, S., Ding, Y., and Guo, J., Adv. Colloid and Interface Science, 78, 197 (1998).

Luttrell, G. H. and Yoon, R.-H., Journal of Colloid and Interface Science, 154(1), 129 (1992).

Mao, L. and Yoon, R.-H., Int. J. Miner. Process. 51(1-4), 171 (1997).

Masliyah, J. S., Chemical Engineering Science 34, 1166 (1979).

Meyer, E.E., Lin, Q., and Israelachivili, J. N., Langmuir, 21, 256 (2005).

Overbeek, J.Th. G., in Colloid Science (ed. by H.R. Kruyt), 1, Elsevier, Amsterdam (1952).

Pazhianur, R. and Yoon, R.-H., Minerals \& Metallurgical Processing, 20(4), 178 (2003).

Israelachvili, J.N. and Parshley, R.M., Nature, 300, 341 (1982).

Pyke, B., Duan, J., Fornasiero, D. and Ralston, J., in Flocculation and Flotation,

Proceedings of the Conference on Flotation and Flocculation: from Fundamentals to Applications, Hawaii, July 28-August 4 (2002).

Rey, D. and Radcliff, G.A., Canadian J. of Chemical Eng. 51, 178 (1973).

Rabinovich, Y. I., and Churaev, N. V., Colloid J. USSR, 41, 392 (1976).

Saffman, P. G. and Turner, J. S., J. Fluid Mech., 143, 16 (1956).

Schubert, H., Int. J. Miner. Process. 56, 257 (1999).

Schulze, H. J., in Physico-Chemical Elementary Processes in Flotation, Elsevier (1984).

Von Sumoluchowski, M., Z. Phys. Chem. 92, 129 (1917)

Sulman, H.L. and Kirkpatrick Pickard, H.F., U.S. Patent 793,808 (1905).

Sundaram, S. and L. R. Collins, Journal of Fluid Mechanics, 335, 75 (1997).

Trahar, W.J., Int. J. Minera. Process, 8, 289 (1981).

Trahar, W.J. and Warren, L.J., Int. J. Minera. Process., 3, 103 (1976).

Yoon, R.-H., Aksoy, B. S., J. Colloid Interface Sci., 211(1), 1 (1999).

Yoon, R.-H.., Flinn, D. H., and Rabinovich, Y. I., J. Colloid Interface Sci. 185(2), 363 (1997).

Yoon, R.-H., and Luttrell, G.H., Mineral Processing and Extractive Metallurgy Review, 5, 101 (1989).

Yoon, R.-H. and Mao, L., J. Colloid Interface Sci., 181(2), 613 (1996).

Yoon, R.-H. and Ravishankar, S. A., J. Colloid Interface Sci., 179(2), 391 (1996).

Williams, J. J. E. and Crane, R. I., International Journal of Multiphase Flow, 9(4), 421 (1983). 
Wang. L. and Yoon, R.-H., Langmuir, 20, 11457 (2004).

Mao, M., Zhang, J., Yoon, R.-H., Ducker, W.A., Langmuir, 20 (5), 1843 (2004).

Trahar, W. J., Int. J. Miner. Process., 8(4), 289-327 (1981).

Trahar, W.J. and Warren, L.J., Int. J. Miner. Process., 3, 103 (1976).

Brake, I., Eldridge, G., Luttrell, G. and Yoon, R.-H., Proceedings of the International

Symposium on Column Flotation, Montreal, Quebec, August 26-28, pp. 13-23 (1996).

Zhang, J., Yoon, R.-H., Mao, M. and Ducker, W.A., Langmuir, under review (2004).

\section{PUBLICATIONS/PRESENTATIONS}

None during the period. 
Appendix C: Studies of Froth Stability and Model Development 


\section{TECHNICAL PROGRESS REPORT}

Contract Title and Number: Crosscutting Technology Development at the Center for Advanced Separation Technologies (DE-FC26-02NT41607)

Sub-Recipient Project Title:

Studies of Froth Stability and Model Development

Principal Investigators:

Roe-Hoan Yoon

Contact Address:

146 Holden Hall

Virginia Polytechnic Institute \& State University

Blacksburg, VA 24061

Subcontractor Address:

\begin{tabular}{|ll|} 
No subcontracts issued. & Phone: \\
& Fax: \\
& E-Mail: \\
\hline
\end{tabular}

Period of Performance:

Starting Date: 9/17/01

Ending Date: 5/30/05

\section{ABSTRACT}

A method to study the stability of three-dimensional foams in the presence of low concentration surfactants has been evaluated. The method involves monitoring the change in pressure external to the foam in container of fixed volume and constant temperature. The experimental data are then converted to decay of the interfacial area of the foam as a function of time based on the equation of state of the foam. We used this technique to investigate the stability of foams stabilized by low concentration sodium dodecyl sulfate (SDS) in the presence of various concentrations of Sodium Chloride $(\mathrm{NaCl})$. It is found that the foams stabilized by $10^{-4} \mathrm{M}$ SDS are unstable with a lifetime of only several seconds. Once the gas supply rate and temperature are under good control, the interfacial area decay curves are sensitive to addition of electrolyte. The results agree well with previous measurements of disjoining pressure isotherm, as well as those obtained using shake tests. This technique is thus expected to be useful for studying the effects of particle size, shape and hydrophobicity on froth stability. 


\section{INTRODUCTION}

\section{Background}

Froths in flotation are a complex system. The stability of froths largely depends on the stability of foams, a simplified system free of solid particles. Many traditional methods exist for the measuring foam stability, such as the Ross-Milles method (1975), Bikerman method (1938), and photographic techniques (Ross and Cutillas, 1955). But none of these techniques is easily reproducible and experimental convenient. The pressure decay method, however, based on the equation of state of foam, provides a way to assess the decay of interfacial area of a foam by monitoring the rate of change in pressure external to the foam in a container of fixed volume at constant temperature. It was originally proposed by Nishioka and Ross (1981), and refined by Yu and Damodaran (1991) and Murray and Liang (1999).

\section{Objective and Approach}

We aimed to evaluate the stability of foams in the presence of low surfactant and electrolyte concentrations.

We used pressure decay method (PDM) to assess foam stability in a reproducible manner. The principle is based on the release of the slight excess Laplace pressure inside a bubble when it collapses. Thus, in a closed container containing foam, the pressure inside the container rises as the foam collapses due to bursting, coalescence, or disproportion of the bubbles, and the pressure rise $(\triangle P)$ is directly related to the decrease in the foam surface $\operatorname{area}(\triangle A)($ Nishioka and Ross, 1981):

$$
3 V \Delta P+2 \gamma \Delta A=0
$$

where $V$ is the total volume of the foam and the vapor space external to the foam, $\gamma$ is the surface tension.

The following equation describes the time-dependent changes in the interfacial area of the foam to changes in the gas pressure external to the foam.

$$
A_{t}=\frac{3 V}{2 \gamma}\left(\Delta P_{\infty}-\Delta P_{t}\right)
$$

A dimensionless fractional interfacial area at any given time $t$ during foam decay was calculated by using the equation

$$
A / A_{0}=\left(\Delta P_{\infty}-\Delta P_{t}\right) / \Delta P_{\infty}
$$

where $A_{0}$ indicates the initial interfacial area of the foam, and $\Delta P_{\infty}$ the final net pressure change. The rate of decrease in $A / A_{0}$ indicates the rate of collapse of the foam, thus the foam stability. 
Below is the schematic diagram for PDM. The right chamber which is identical to the left serves as a reference chamber to minimize the temperature fluctuation. Nitrogen gas saturated with water was used to produce the foam, using a constant flow rate of 0.6 liter per minute. Nitrogen was bubbled through the sample solution via these 12 needles for $30 \mathrm{sec}$ before valves were closed and the pressure difference between the sample and reference chambers monitored.

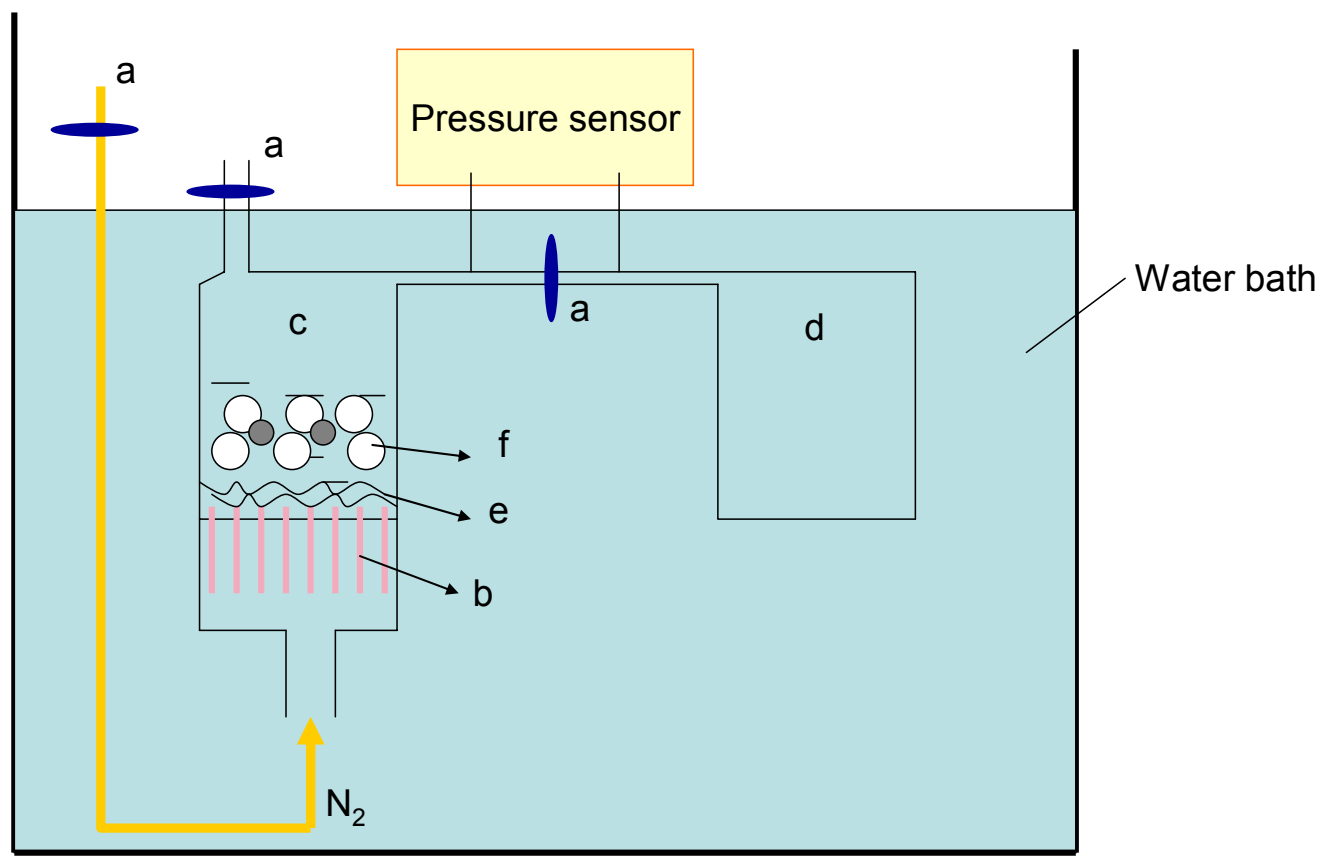

Figure 1. Schematic drawing of pressure decay method. Valves (a), 12 stainless steel needles with I.D. of $0.1 \mathrm{~mm}$ (b), cylindrical glass sample chamber (c), cylindrical glass reference chamber (d), surfactant solution in sample chamber (e), foams or froths (f).

Preliminary test showed that the gas flow rate and temperature should be under good control for unstable foams in the presence of low concentration surfactants.

\section{PROJECT TASKS}

\section{Task 1 - Foam/Froth Studies}

For comparison, we used a crude method of measuring the foam stability, the shake tests (Waltermo et al., 1996). The foam stability was measured using $50 \mathrm{ml}$ volumetric flasks filled with $25 \mathrm{ml}$ of surfactant solution. Each sample was shaken by hand with fixed frequency and time, then left to stand still. The decay time of the foam was recorded as the 
time before the appearance of the periphery of a clear surface area in the center of the foam, which eventually expanded onto a foam-free liquid surface.

Table 1. the lifetime of the foams stabilized by $10^{-4} \mathrm{M}$ SDS in the absence or presence of $\mathrm{NaCl}$.

\begin{tabular}{lccccc}
\hline $\mathrm{NaCl}(\mathrm{M})$ & 0 & $4 \times 10^{-4}$ & $10^{-3}$ & 0.01 & 0.1 \\
\hline $\mathrm{T}(\mathrm{sec})$ & 32 & 89 & 162 & 128 & 65 \\
\hline
\end{tabular}

Table 1 shows that the foam lifetime ( $\mathrm{T}$ ) increased from 32 to 162 seconds as $\mathrm{NaCl}$ concentration was increased from 0 to $1 \mathrm{mM}$. This is probably due to the decrease in hydrophobic force in single foam films. As $\mathrm{NaCl}$ concentration was increased to as high as $0.1 \mathrm{M}$, the foam lifetime decreased, indicating that the high concentration electrolyte lead to the decrease in the double layer repulsion, which in turn makes the foam less stable.

To assess foam stability more exactly and reproducibly, we used the pressure decay method. The results were shown in figure 2.

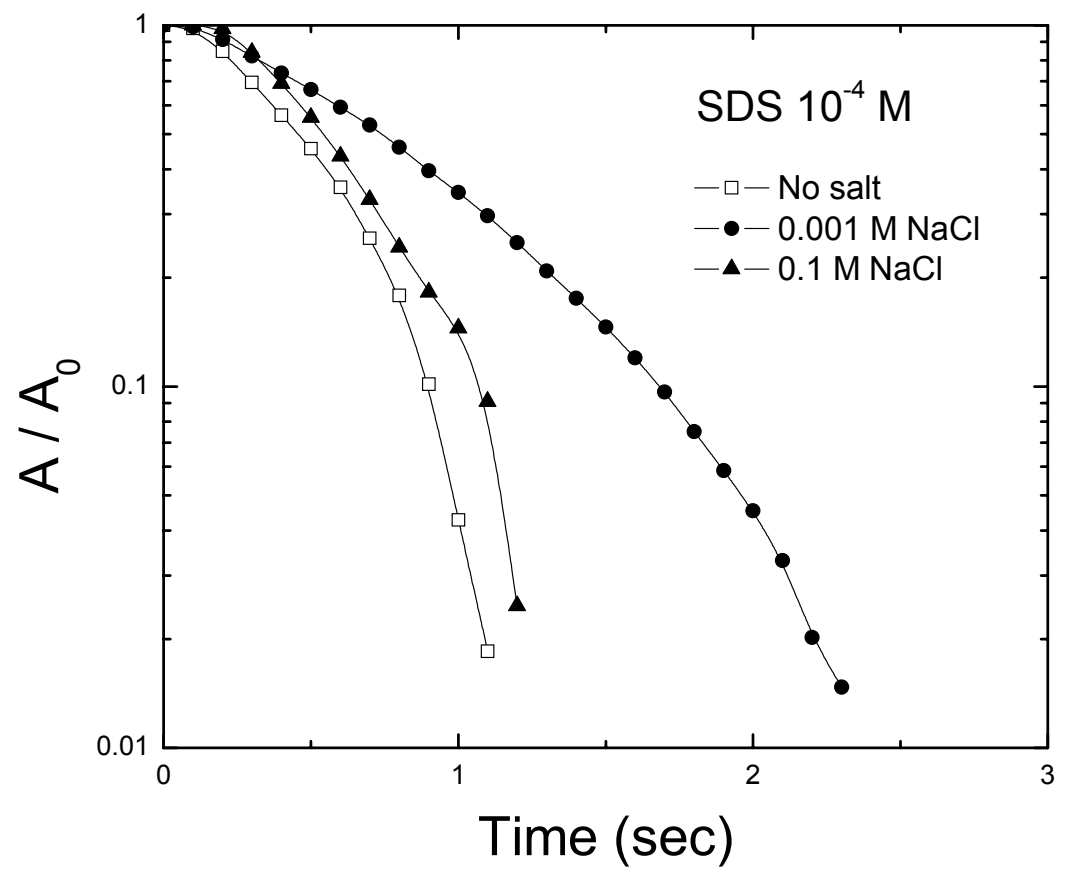

Figure 2 Surface area decay of SDS foams containing various concentrations of $\mathrm{NaCl}$. 
Figure 2 shows that the SDS foams are unstable and decay thoroughly in several seconds. The foam containing $0.001 \mathrm{M} \mathrm{NaCl}$ is less unstable, probably due to the reason that the attractive hydrophobic force in lamella of the foams is sufficiently suppressed by the electrolyte. For single foam films containing $10^{-4} \mathrm{M}$ SDS with no $\mathrm{NaCl}$, there is a strong hydrophobic force with $\mathrm{K}_{232}=2.3 \times 10^{-18} \mathrm{~J}$, much larger than van der Waals attraction (Hamaker constant $3.7 \times 10^{-20} \mathrm{~J}$ ). This result is consistent with the measurements of the disjoining pressure isotherms for single foam films reported in Wang and Yoon (2004). At high $\mathrm{NaCl}$ concentration, i.e. $0.1 \mathrm{M}$, the double layer repulsion was efficiently suppressed, so that the foam became less stable.

Combing Table 1 and Figure 2, one can see that PDM is suitable for measuring the stability of foams formed from low surfactant concentrations, which is the case for froth flotation. As for froths, involving solid particles, we expect this method is preferable since only the pressure external the froth will be monitored. If other methods were used, either the bubble formation is not reproducible, or the solid particles bring unexpected disturbance to optical and other properties of the sample.

\section{SUMMARY}

The pressure decay method was evaluated for studying the stability of foams and froths at low surfactant concentrations. It involves monitoring the change in pressure external to the foam in container of constant volume and temperature. The experimental data are then converted to decay of the interfacial area of the foam as a function of time. The measured results agree well with previous measurements of disjoining pressure isotherm, as well as those obtained using shake test. This technique is thus expected to be useful for studying the effects of particle size, shape and hydrophobicity on froth stability.

\section{FUTURE WORK}

Using pressure decay method to investigate froth stability.

\section{REFERENCES}

A. Waltermo, P.M. Claesson, S. Simonsson, E. Manev, I Johansson, V. Bergeron, Langmuir, , 12 (1996) 5271.

“1975 Annual book of ASTM Standards, “ D1173-53, Part 30, p. 180.

J.J. Bikerman, Trans. Faraday. Soc. 34 (1938) 634.

S. Ross, M.J. Cutillas, J. Phys. Chem. 59 (1955) 863.

G. Nishioka, S. Ross, J. Colloid Interface Sci., 81 (1981) 1 
M.-A. Yu, S. Damodaran, J. Agric. Food Chem. 39 (1991) 1555

B.S. Murray, H.-J. Liang, J. Agric. Food Chem. 47 (1999) 4984

L. Wang and R.-H. Yoon, Langmuir, in press.

\section{PUBLICATIONS/PRESENTATIONS}

To date, no major publications have resulted from this project.

\section{APPENDICES}

No appendices are included in this report. 
Appendix D: Direct Measurement of Forces in Flotation Systems 


\section{TECHNICAL PROGRESS REPORT}

\begin{tabular}{|c|c|c|}
\hline Contract Title and Number: & \multicolumn{2}{|c|}{ Period of Performance: } \\
\hline$\overline{\text { Establishment of the Center for Advanced }}$ & \multirow{2}{*}{$\begin{array}{l}\text { Starting Date: } \\
\text { Ending Date: }\end{array}$} & $9 / 17 / 01$ \\
\hline Separation Technologies (DE-FC26-01NT41091) & & 05/31/05 \\
\hline Sub-Recipient Project Title: & \multicolumn{2}{|c|}{ Report Information: } \\
\hline \multirow[t]{3}{*}{ Direct Measurement of Forces in Flotation Systems } & Type: $\quad A$ & Annual \\
\hline & Number: & 5 \\
\hline & Period: & $5 / 17 / 04-9 / 30 / 04$ \\
\hline Principal Investigators: & Date: & $11 / 30 / 04$ \\
\hline William Ducker and Roe-Hoan Yoon & Code: & VA004 \\
\hline Contact Address: & \multicolumn{2}{|c|}{ Contact Information: } \\
\hline 304 Davidson Hall and 146 Holden Hall & Phone: $\quad 5$ & $540-231-2249$ \\
\hline Virginia Tech & Fax: & \multirow[b]{2}{*}{ cast@vt.edu } \\
\hline Blacksburg VA & E-Mail: & \\
\hline Subcontractor Address: & \multicolumn{2}{|c|}{ Subcontractor Information: } \\
\hline No subcontracts issued. & \multicolumn{2}{|c|}{ Phone: } \\
\hline
\end{tabular}

\section{ABSTRACT}

The objective of this project is to directly measure the interaction force between a particle and bubbles as a function of separation distance. This force controls the attachment and detachment of particles to bubbles, which is an essential step in determining the efficiency of a flotation process. A specially designed device will be fabricated for these measurements. The device will use the force-detection method employed by an Atomic Force Microscope and the separation-detection method employed by a Surface Forces Apparatus. The key advances are to explicitly measure both the separation between the particle and the bubble and the shape of the bubble at all times. Without this separation and shape data, it would be difficult to relate the measured forces to flotation results. After fabrication of the device, measurements of the interaction forces acting on hydrophilic, hydrophobic, and charged particles in aqueous solutions of surfactant molecules will be obtained. During this reporting period, force measurements were conducted using a model system (silica particle and plate).

\section{INTRODUCTION}

\section{$\underline{\text { Background }}$}

Froth flotation is the most important solid-solid separation process for upgrading run-of-the-mine (ROM) ores and coal (Leja, 1982). In froth flotation, a stream of small air bubbles is introduced to the bottom of a tank (or flotation cell) in which a finely 
ground ore is suspended in water. The air bubbles rise to the top of the tank under a buoyancy force, and in doing so, they collide with suspended particles. Purification of the ore is based on differences in the attachment and/or detachment of particles to the bubbles. It is believed that the attachment of particles to a bubble surface is caused by a "hydrophobic" interaction. Several investigators have attempted to directly measure the forces between air bubbles and particles, but the results are not consistent with experience from flotation practice. The main difficulty lies in the fact that bubbles deform during in the interaction. This deformation leads to confusion as to the real separation between a particle and a bubble. Knowledge of the separation is vital to elucidate the range of the force, and thus the mechanism of the interaction. The range of the force is a required input for calculation of the kinetics of flotation.

\section{Objective and Approach}

The overall objective of this project is to perform direct measurements of forces acting on different types of particles when they collide with bubbles. Data from these measurements make it possible to determine the relative importance of long-range and short-range forces in flotation and to examine the role of collectors (surfactants) in controlling these forces. In particular, the data make it possible to develop a better understanding of the nature of the "hydrophobic" interaction that is thought to be responsible for the capture of particles by bubbles.

\section{PROJECT TASKS}

\section{$\underline{\text { Task } 1 \text { - Design and Fabrication }}$}

The apparatus used to experimentally measure the surface forces between to interacting surfaces was described in the last technical progress report. The apparatus uses the deflection of a micro-fabricated spring to determine the surface force, while the separation distance is indirectly measured using an interferometer. No additional construction work was required during the past reporting period.

\section{$\underline{\text { Task } 2 \text { - Experimental Measurements }}$}

Several series of surface force experiments were conducted during this reporting period using quartz particles and plates. The surface forces were measured at at room temperature $\left(\sim 25^{\circ} \mathrm{C}\right)$ using the colloidal probe technique developed by Ducker et al. (1991, 1992). The hydrophobicity of the surfaces was controlled by treating with surfactant $\left(\mathrm{C}_{18} \mathrm{TACl}, 97 \%\right.$ purity). To remove contamination, the quartz surfaces were boiled in a $\mathrm{H}_{2} \mathrm{SO}_{4} / \mathrm{H}_{2} \mathrm{O}_{2}$ solution for 20 minutes, rinsed thoroughly with nanopure water, equilibrated with the nanopure water in a sealed vial for at least 30 minutes, and then

used for the experiments. When required, the surfactant solutions were degassed using the procedure described by Sakamoto et al. (2002). 
Figures 1 and 2 show the results of our measurement of the forces between a glass particle and a flat silica plate in aqueous $\mathrm{C}_{18} \mathrm{TACl}$ solution in solutions that were equilibrated with air, and in solutions that were degassed. The experimental data show (i) that the most attractive forces are much greater in magnitude than the van der Waals force and (ii) the effect of degassing is not very large. When the concentration of surfactant is increased, first the repulsive force decreases, then becomes strongly attractive, then becomes repulsive again. The overall trend is the same for both the air-equilibrated and degassed solutions; there is just a shift in the concentration at which a particular force occurs.

A possible explanation for the effect of degassing is that it changes the $\mathrm{pH}$ of the solution. The air-equilibrated solution was found to have a $\mathrm{pH}$ of about 5.7, whereas the $\mathrm{pH}$ of the degassed solution was about 6.6. The change in $\mathrm{pH}$ may be responsible for the change in forces since it is well known that an increase in $\mathrm{pH}$ causes an increase in the dissociation of surface silanol groups, leading to a greater charge on the solid. Also, Goloub et al. (1996) showed that a greater charge on the solid leads to an increase in the amount of adsorbed surfactant for a given concentration near where the point where the net charge on the surface (solid plus surfactant) is approximately zero. Thus, in the degassed solution, the $\mathrm{pH}$ is a little higher and the forces are shifted a little to lower surfactant concentrations. The overall trend of forces in air-equilibrated and degassed solutions is similar, but owing to the large gradient of the force with respect to concentration, there can be large effects at some individual concentrations due to degassing. For example, in $10^{-5} \mathrm{M} \mathrm{C}_{18} \mathrm{TACl}$, the force is attractive in air-equilibrated solution, and repulsive in degassed solution. The difference may simply be a shift in the amount of adsorbed surfactant as a result of the $\mathrm{pH}$ change.

To further investigate the effects of charging, the surface force was measured as a function of $\mathrm{pH}$ in $10^{-5} \mathrm{M} \mathrm{C}_{18} \mathrm{TACl}$ in solutions that were not degassed. The $\mathrm{pH}$ was 
altered via the addition of $\mathrm{NaOH}$. The data given in Figure 3 shows that addition of $\mathrm{NaOH}$ produces an effect that is similar to increasing the concentration of surfactant. In particular, a shift in the $\mathrm{pH}$ from 5.7 to 6.5 produces a similar change to that of degassing.

\section{$\underline{\text { Task } 3 \text { - Data Analysis }}$}

There are several possible explanations for the anomalous attractive force observed in the experimental measurements. These include (1) adsorbed air-bubbles, (2) attractive double-layer forces, (3) disturbed water structure, and (4) correlations between adsorbed

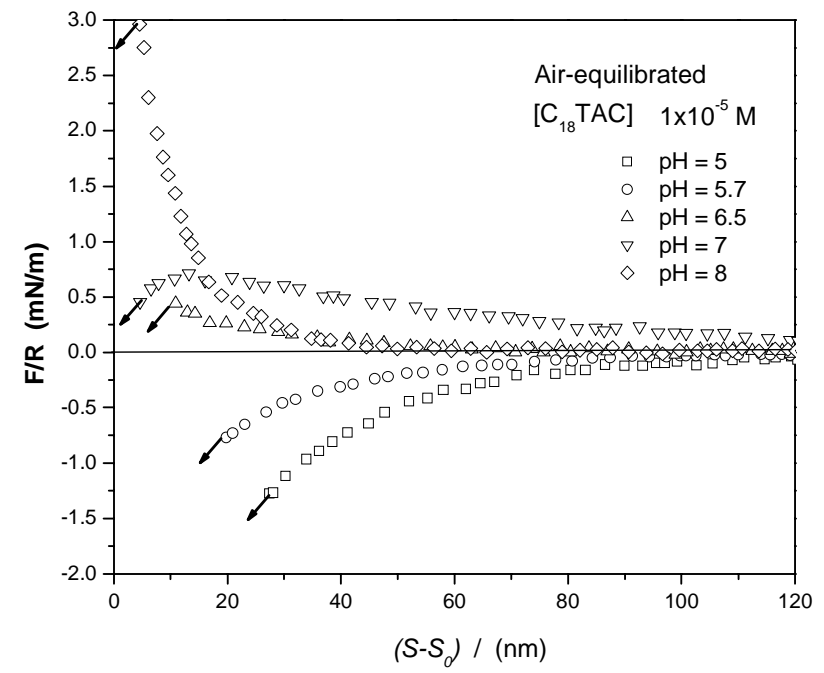

Fig. 3. Effect of $\mathrm{pH}$ on forces in air-equilibrated solution at a constant surfactant concentration of $1 \times 10^{-5} \mathrm{M}$. patches.

Adsorbed air-bubbles. The data show that the effect of degassing is both slight, and explicable in terms of $\mathrm{pH}$. There were no discontinuities (steps) in the force curves that are often used as a signature for the air-bubbles. Both changes on degassing and the existence of steps have been used to support the adsorbed bubble hypothesis, but of course, their absence does not directly show that adsorbed bubbles are not present. However, it is difficult to explain the observed loss of the attractive force with an increase in $\mathrm{pH}$ in terms of the adsorbed bubble hypothesis. It is not clear why an increase in $\mathrm{pH}$ would cause the removal of the bubbles. As such, there is no strong evidence to suggest that adsorbed bubbles are responsible for the strong attractive force.

Attractive double-layer forces between two different homogeneous surfaces. The measured attractive forces are approximately exponential with respect to separation. Also, the two solids are different: the particle is glass whereas the plate is silica. A different charge on each surface offers the possibility of attractive double-layer forces. To examine this effect, the zeta potential of both the particles and plate (after grinding it into small particles) were measured. Although the potentials were found to be slightly different (Jiang, 2004), the difference in potentials is much too low to explain the magnitude of the measured attractive forces.

Disturbed water structure. The original explanations for the long-ranged attractive force between high water-contact-angle solids was that water is an unfavorable state in the thin film between two alkane surfaces because of the inability of water to hydrogen bond to the surfaces i.e. a larger-scale version of the hydrophobic effect. The problem with this explanation is that the range of the forces (in our case $>80 \mathrm{~nm}$ or about 300 water diameters) is very large compared to the range of normal hydration effects (a 
few water diameters). Also, the solids are not particularly hydrophobic, i.e., the static contact angle of a $10^{-5} \mathrm{M} \mathrm{C}_{18} \mathrm{TACl}$ solution on the silica plate is about $50^{\circ}$ (droplet on plate) to $60^{\circ}$ (bubble on plate). The only evidence for the disturbed water hypothesis is that the attractive force starts to diminish at about the same concentration that the zeta potential begins to be positive. A positive zeta potential is also an indication that additional surfactant adsorbs with the headgroups facing solution (i.e. decreasing the hydrophobic nature of the surface).

Heterogeneous surfaces. It has been proposed that anomalous long-range attractive forces between surfactant-coated surfaces may arise because of correlations between patches on two heterogeneous surfaces. Ellipsometry and AFM studies indicate that the surfactant is slow to adsorb and that the adsorption proceeds via patches. Miklavic et al. (1994) examined the case when a surface contains an equal number of mobile positive and negative charge patches and found that the force is attractive, exponential, and dependent on the salt concentration. At low salt concentration, the decay length scales with the patch size, and at high salt concentration, the decay length is given by the solution Debye-length. To test whether the forces are electrostatic in origin, the forces were measured as a function of salt concentration in $5 \times 10^{-6} \mathrm{M} \mathrm{C}_{18}$ TACl. This is the concentration where the attractive force is a maximum and the zeta potential is approximately zero. The resultant data show that the decay length of the force decreases as salt is added. In fact, the decay length was found to be similar to the Debye length at moderate salt concentrations, as predicted by Miklavic's model. Therefore, one can imagine two scenarios for the formation of the charge patches with correlated positions. In the first scenario, they are formed on the isolated surface due to the slow rate of adsorption and the strong mutual attractive of the surfactant alkyl chains in water. The patches must be mobile to allow correlations between positive and negative patches. In the second scenario, they are formed when surfactant layers on two separate surfaces are brought into contact and then separated. During separation, the rupture occurs in the plane between the surfactant and the solid.

\section{SUMMARY}

During the past reporting period, experimental measurements of surface forces were performed using model (silica) surfaces treated with surfactant $\left(\mathrm{C}_{18} \mathrm{TACl}\right)$. The surfactant produces attractive forces between silica-like surfaces in aqueous solutions. These attractive forces are much greater in magnitude and range than expected for the van der Waals force between silica or hydrocarbon surfaces. Both the magnitude and range of the anomalous attractive forces are diminished through the addition of low concentrations of salt (10-4 M NaCl), but are not largely influenced by degassing or $\mathrm{pH}$ changes. In the absence of a good reason to believe that either the adsorption of bubbles or the water structure is dramatically affected by such low concentrations of simple salt or small changes in $\mathrm{pH}$, several other possibilities have been considered to explain the long-range attraction. An explanation that is consistent with the experimental results is that the attractive force is due to correlations between patches of positive charge on one surface and patches of negative charges on the other surface. Such patches could exist on isolated surfaces and the correlations be produced by surface mobility, or patches of 
opposite charge on each surface could be produced by the fusion and rupture of layers when the surfaces are brought into contact and then separated.

\section{FUTURE WORK}

Much of the work conducted during the next reporting period will focus on the continued construction and shakedown testing of the experimental apparatus for the direct measurement of force and separation distance between bubbles and particles.

\section{REFERENCES}

Ducker, W. A., Senden, T. J. and Pashley, R. M. 1991. Direct Measurement of Colloidal Forces Using an Atomic Force Microscope Nature, 353: 239-241.

Ducker, W. A., Senden, T. J. and Pashley, R. M. 1992. Measurement of Forces in Liquids Using a Force Microscope Langmuir, 8: 1831-1836.

Goloub, T. P., Koopal, L. K., Bijsterbosch, B. H. and Sidorova, M. P. 1996. Adsorption of Cationic Surfactants on Silica. Surface Charge Effects. Langmuir, 12: 3188-3194.

Jiang 2004 et al Submitted to Langmuir.

Leja, J., 1982. Surface Chemistry of Froth Flotation, Plenum Press, New York, N.Y.

Miklavic, S. J., Chan, D. Y. C., White, L. R. and Healy, T. W. 1994. Double-Layer Forces between Heterogeneous Charged Surfaces Journal of Physical Chemistry, 98: 9022-9032.

Sakamoto, M., Kanda, Y., Miyahara, M. and Higashitani, K. 2002. Origin of Long-Range Attractive Force between Surfaces Hydrophobized by Surfactant Adsorption Langmuir, 18: 5713-5719.

\section{PUBLICATIONS/PRESENTATIONS}

To date, no major publications have resulted from this project.

\section{APPENDICES}

No appendices are included in this report. 
Appendix E: Novel Surfactants as Collectors for Froth Flotation 


\section{TECHNICAL PROGRESS REPORT}

\begin{tabular}{|c|c|}
\hline Contract Title and Number: & Period of Performance: \\
\hline Establishment of the Center for Advanced Separation & Starting Date: $\quad$ March 1, 2003 \\
\hline Technologies (DE-FC26-01NT41091) & Ending Date: \\
\hline
\end{tabular}

Sub-Recipient Project Title:

NOVEL SURFACTANTS AS COLLECTORS FOR FROTH FLOTATION.

Principal Investigators:

Richard D. Gandour

Contact Address:

Department of Chemistry, MC 0212

Virginia Tech

Blacksburg, VA 24061

Subcontractor Address:

No subcontracts issued.
Report Information:

Type: Annual

Number: 3

Period: $\quad 10 / 1 / 03-9 / 30 / 04$

Date: $\quad 1 / 5 / 2005$

Code: VA007-R03

Contact Information:

Phone: 540-231-3731

Fax: $\quad$ 540-231-3255

E-Mail: gandour@vt.edu

Subcontractor Information:

Phone:

Fax:

E-Mail:

\section{ABSTRACT}

The three series of hydra surfactants have been synthesized and characterized. All, including those with 22 carbons in the chain, are quite soluble in triethanolamine solutions

\section{INTRODUCTION}

\section{Background}

Froth flotation involves separating finely divided solid particulates suspended in water.(Fuerstenau and Herrera-Urbina, 1989) Many mining companies worldwide employ froth flotation to enhance the concentration of desired mineral and coal fines. Processing occurs on the scale of billions of tons of ore annually and consumes millions of kilograms of reagentscollectors, depressants, activators, modifiers, and frothers. The proposed hydra surfactants will function as collectors.

\section{Objective and Approach}

Our goals are to synthesize novel, inexpensive surfactants that outperform current collectors in mineral flotation. These surfactants will selectively bind to mineral particles and create a 
hyrdophobic coating on a particle to enable selective separation by froth flotation. We plan to synthesize surfactants that have very hydrophobic, long chains, yet have sufficient water solubility for mineral processing. These amphiphiles, which we call 'hydra surfactants', will have one tail and three identical functional groups on the head. These novel molecules will be useful for many different separations in mineral processing.

We expect the hydra surfactants to enhance collector properties in two critical areas: (1) stronger binding to the particle through multi-valent attachment and (2) increasing the solubility for longer chains. First, they should improve binding of the surfactant to the particle because multiple functional groups increase the chances for attachment. After the initial attachment, it is easier for the remaining functional groups to attach. Second, the increased polarity of the head group will increase the water solubility of very long hydrophobic chains. Consequently, fewer bound molecules will be needed to produce the desired hydrophobicity. Improvement in both critical areas will ultimately produce a method that uses a smaller amount of collector while achieving a better separation

\section{PROJECT TASKS}

\section{Task 1. Description of Synthesis.}

During the past 6 months, we have spent most of our efforts on the synthesis of hydra surfactants. Our research team of three graduate students, one laboratory specialist, and two undergraduate are making progress.

We recently completed the syntheses of the single-tailed, tri-headed amphiphiles. (Scheme 1) The condensation of Behera's amine (1) with either an acid chloride or a carboxylic acid that was activated by DCC afforded the triesters (3EAm\#) in good-to-moderate yields (step iv) of purified products. In general, the acid chloride reaction gave higher yields than the DCC method. Formolysis of the triesters produced the triacids, 3CAm\#, in high yields of recrystallized products. Syntheses of the other series began with Weisocyanate ${ }^{\mathrm{TM}}(2)$. Condensation of longchain amines with 2 occurred at room temperature to afford good-to-high yields (step v) of 3EUr\#. Formolysis of the triesters produced the triacids, 3CUr\#, in good yields of recrystallized products. Condensation of long-chain alcohols with 2 occurred at $95{ }^{\circ} \mathrm{C}$ with triethylamine (step vi) as the solvent to afford good isolated (after flash chromatography) yields of $\mathbf{3 E C b \# . ~}$

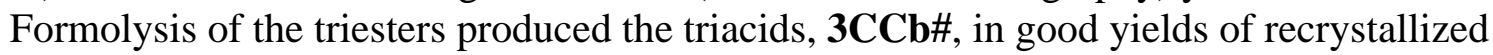
products. All compounds were fully characterized.

Solubility studies are progressing. To date, we are studying the solubility of these amphiphiles in aqueous triethanolamine solutions. As shown for $N$-lauroyl-L-glutamate/water, triethanolammononium salts can be dissolved in a much greater concentration than potassium salts. Compounds 3CAm13, 3CAm 17, and 3CAm 21 readily dissolve to form $4.0 \times 10^{-2}-M$ solutions of each amphiphile. Compounds 3CUr14 and 3CAm 18 readily dissolve to form $4.0 \times$ $10^{-2}-M$ solutions of each amphiphile; at this concentration, 3CUr22 forms a cloudy solution in an hour after initially dissolving. Mindful that chain length can affect the $p K_{\mathrm{a}}$ in fatty acids because of aggregation, we have found that a $4.0 \times 10^{-2}-M$ solution of 3 CUr22 can be made in a $4 \%$ triethanolamine in water. We expect that these concentrations are well above those needed for flotation. 
Scheme 1. i. $\mathrm{BnN}^{+} \mathrm{Me}_{3} \mathrm{OH}$, DME, $70-80^{\circ} \mathrm{C}, 1$ h (73\%); ii. $\mathrm{H}_{2}$, Raney Ni, EtOH, 38-40 ${ }^{\circ} \mathrm{C}$, 2 h (90\%); iii. ( $t$ BuOCO) ${ }_{2} \mathrm{O}$, DMAP, $\mathrm{CH}_{2} \mathrm{Cl}_{2}, 15$ min; iv. $\mathrm{R} \mathrm{CH}_{2} \mathrm{COCl}, \mathrm{PhH}, \mathrm{Et}_{3} \mathrm{~N}$, $42 \mathrm{~h}(80 \%)$ or $\mathrm{R} \mathrm{CH}_{2} \mathrm{COOH}$, DCC, HOBT, THF, 48-72 h (61-82 \%); v. R1- $\mathrm{NH}_{2}, \mathrm{CH}_{2} \mathrm{Cl}_{2}$, rt, 18 h (82-92\%); vi. R1-OH, $\mathrm{NEt}_{3}, 95{ }^{\circ} \mathrm{C}$, 4 h (65-85\%); vii. HCOOH, THF, 18-48 h (55-80 \%). 3CAm\#:R1 = $n-\mathrm{C}_{\mathrm{n}} \mathrm{H}_{2 \mathrm{n}+1}, \mathrm{n}=12,14,16,18,20,22$ and \# = 13, 15, 17, 19, 21, 23; For 3CUr\#:R1 $=n-C_{n} H_{2 n+1}$, $n$ and $\#=14,16,18,20,22$; For 3CCb\#:R1 $=n-C_{n} H_{2 n+1}, n$ and $\#=14,16,18,20$, 22

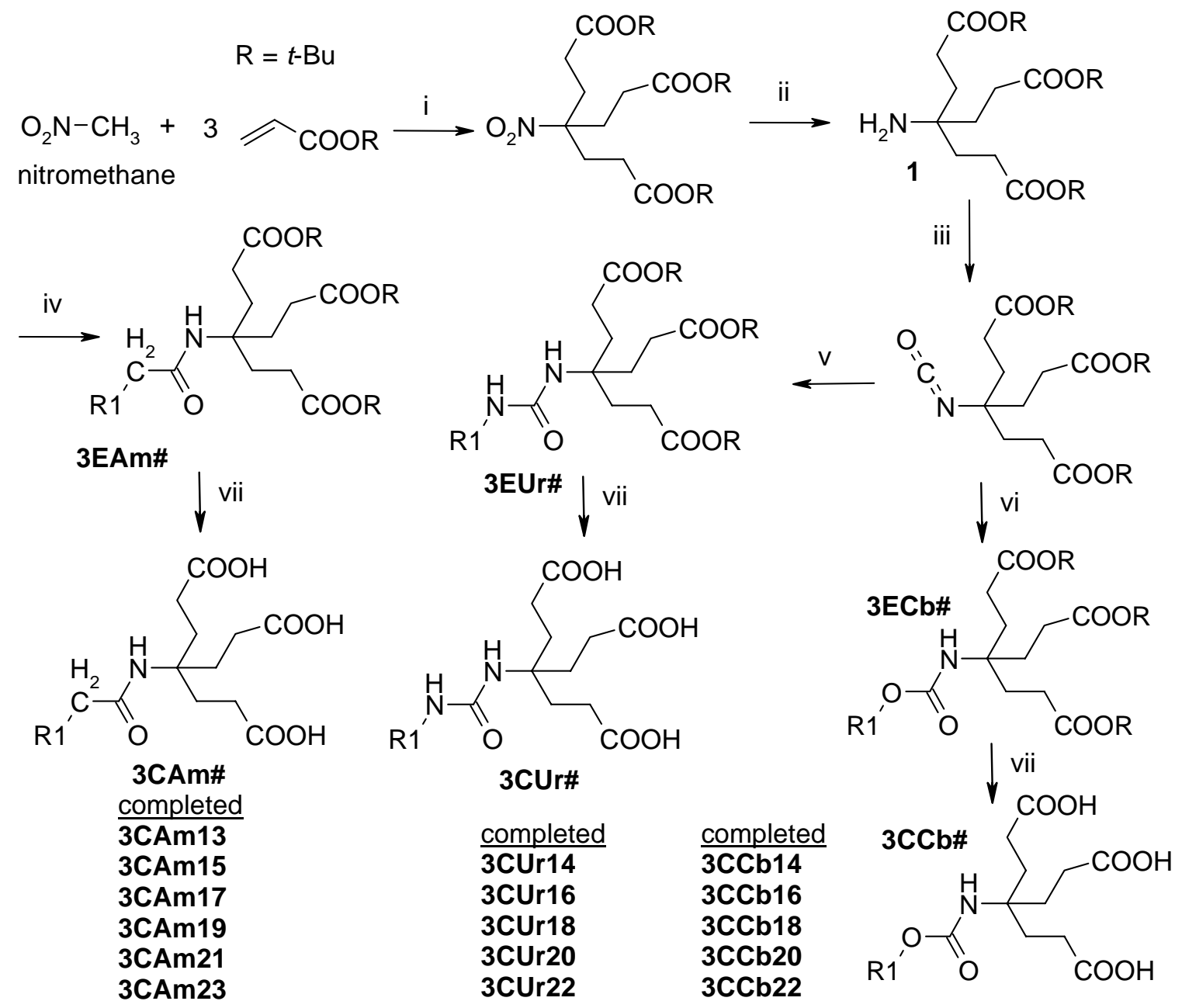

\section{SUMMARY}

We have developed a procedure for making three classes of hydra surfactants. We have that they are soluble in aqueous triethanolamine.

\section{FUTURE WORK}

We shall scale up these syntheses to provide samples for testing. We shall collaborate with Professor Yoon's group to test the novel surfactants in flotation tests. 


\section{REFERENCES}

Fuerstenau, D.W. and Herrera-Urbina, R., 1989. Flotation Reagents. In: S. Chander and R.R. Klimpel (Editors), Advances in Coal and Mineral Processing Using Flotation. Society for Mining, Metallurgy, and Exploration, Inc., Littleton, Colorado, pp. 3-18.

\section{PUBLICATIONS/PRESENTATIONS}

None to report during this period. 
Appendix F: Column Flotation of Fine Dolomitic Phosphate by Selective Fatty Acids 


\section{TECHNICAL PROGRESS REPORT}

\begin{tabular}{lll} 
Contract Title and Number: & \multicolumn{2}{l}{ Period of Performance: } \\
\cline { 2 - 2 } Establishment of the Center for Advanced Separation & Starting Date: & 9/17/01 \\
Technologies (DE-FC26-01NT41091) & Ending Date: & $5 / 31 / 05$
\end{tabular}

Sub-Recipient Project Title:

Column Flotation of Fine Dolomitic Phosphate by

Selective Fatty Acids

Report Information:

Type: Annual

Number: 2

Principal Investigators:

Period: $\quad 10 / 1 / 03-09 / 30 / 04$

Felecia Peng

Date: $\quad 9 / 30 / 04$

Code: WV006-R02

Contact Address:

Contact Information:

365 Mineral Resources Building

Phone: 304-293-7680

Mining Engineering Dept., West Virginia University

Fax: $\quad 304-293-5708$

Morgantown, WV 26506-6070

E-Mail: ffpeng@mail.wvu.edu

Subcontractor Address:

Subcontractor Information:

No subcontracts issued.

Phone:

Fax:

E-Mail:

\section{ABSTRACT}

Based on the combined results obtained from stirred tank cell flotation for reagent requirement tests, and initial fine dolominitc phosphate pebble column flotation tests, four major operation variables which affect the separation performance of fine dolomitic phoaphate pebble flotation significantly are determined. The variables are air flowrate, $\mathrm{pH}$, collector dosage and column interphase height. Center Composite Experiment Design are selected for systematic testing fine dolomitic phosphate pebble column flotation. Some of the experiment results obtained in this period are reported. The overall $\mathrm{P}_{2} \mathrm{O}_{5}$ recovery is very high, but the rejection of $\mathrm{MgO}$ is not so high as expected due to the reagent dosages are low for this period of the experiment design runs. The improvement in rejection of $\mathrm{MgO}$ is expecting for the forth coming experiment design runs.

\section{INTRODUCTION}

Background:

Development of $\mathrm{MgO}$ removal process are important for recover phosphate from low grade phosphate ores, and existing dolomitic phosphate pebbles rejects stockpiled at mine sites in Florida. The processes so far developed can not produce the acceptable phosphate concentrate with low $\mathrm{MgO}$ content $(<=1 \% \mathrm{MgO})$. The phosphate concentrate with high content of $\mathrm{MgO}$ is detrimental for the down stream filtration process in phosphoric acid manufacturing process for making fertilizer. Recent newly developed selected fatty acid 
collectors extracted from cotton seeds, and synthesized collectors show they are the effective dolomite collectors in reverse fine dolomitic phosphate flotation process (Hwang, et al., 2003). Preliminary test results in WVU Mining Engineering Department for fine dolomitic phosphate pebble in column flotation showed that PA-31 is an effective collector for dolomite flotation. The results also show that the high recovery of $\mathrm{P}_{2} \mathrm{O}_{5}$ and low $\mathrm{MgO}$ in the phosphate product can be achieved with this improved process.

\section{Objective and Approach}

The objective of this project is to use the selective fatty acid soap collector, PA-31 or its substitute for fine dolomitic phosphate pebble flotation in the flotation column. The depressant used for phosphate particles is the mixture of phosphoric acid, or the mixture of phosphoric acid and sulfuric acid. Based on the Central Composite Experiment Design the major operation variables affect the column flotation results in terms of $\mathrm{P}_{2} \mathrm{O}_{5}$ recovery and $\mathrm{MgO}$ rejection will be conducted and analyzed in systematic approach.

\section{PROJECT TASKS}

In this period, the reagent dosages required for column flotation tests were determined in the 2-Liter stirred-tank cell in a D-12 model Denver flotation machine. For initial column flotation tests, combination of one-16th fraction of the $2^{7}$ and a resolution II design with which only main effect can be estimated (similar to Plackett-Burman Experiment Design), is used to test on some dolomitic phosphate pebble samples. The results of reagent tests combined with the results of the screening experiment design runs for the initial column flotation, the operation conditions for the column flotation were determined as shown in Table 1.

Table 1 Conditions for the column flotation of fine dolomitic phosphate pebbles

\begin{tabular}{|l|c|c|c|}
\hline Factors & $\mathbf{- 1}$ & $\mathbf{0}$ & $+\mathbf{1}$ \\
\hline $\mathrm{pH}$ & 4.5 & 5 & 5.5 \\
\hline Collector & 2 & 2.5 & 3 \\
\hline Interface $(\mathrm{cm})$ & 10 & 15 & 20 \\
\hline Air rate $(\mathrm{ml} / \mathrm{min})$ & 3.5 & 4 & 4.5 \\
\hline
\end{tabular}

Central composite experiment design is applied for the experiment runs based on the information provided in Table 1. The distance from the center of the design space to a factorial point is \pm 1 unit for each factor. The distance from the center of the design space to a star point is $\pm \alpha$. In this case, we select $\alpha=1$, then the design is called face centered composite design. Based on the output table of the statistics software JMP, 26 runs are needed for the analysis. The coded conditions and natural conditions are arranged as shown in Table 2. The column flotation of fine phosphate pebble phosphate pebble has been tested. Some of the experiment design runs results completed is given in Table 3. Figure 1 also shows the relationship between $\mathrm{P}_{2} \mathrm{O}_{5}$ recovery and $\mathrm{MgO}$ recovery from the test results. 
Table 2 Central composite experiment design

\begin{tabular}{|c|c|c|c|c|c|c|c|c|c|c|c|c|c|c|c|c|c|}
\hline \multirow[t]{2}{*}{ Run } & \multicolumn{4}{|c|}{ Coded conditions } & \multicolumn{4}{|c|}{ Natural conditions } & \multirow[t]{2}{*}{ Run } & \multicolumn{4}{|c|}{ Coded conditions } & \multicolumn{4}{|c|}{ Natural conditions } \\
\hline & pH & CL & IF & AR & pH & CL & IF & AR & & CL & IF & AR & $\mathbf{p H}$ & CL & IF & AR & pH \\
\hline 1 & -1 & -1 & -1 & -1 & 4.5 & 2 & 10 & 3.5 & 14 & 1 & -1 & 1 & 1 & 5.5 & 2 & 20 & 4.5 \\
\hline 2 & 1 & -1 & -1 & -1 & 5.5 & 2 & 10 & 3.5 & 15 & 1 & 1 & -1 & 1 & 5.5 & 3 & 10 & 4.5 \\
\hline 3 & -1 & 1 & -1 & -1 & 4.5 & 3 & 10 & 3.5 & 16 & 1 & 1 & 1 & 1 & 5.5 & 3 & 20 & 4.5 \\
\hline 4 & -1 & -1 & 1 & -1 & 4.5 & 2 & 20 & 3.5 & 17 & 1 & 0 & 0 & 0 & 5.5 & 2.5 & 15 & 4 \\
\hline 5 & -1 & -1 & -1 & 1 & 4.5 & 2 & 10 & 4.5 & 18 & -1 & 0 & 0 & 0 & 4.5 & 2.5 & 15 & 4 \\
\hline 6 & 1 & 1 & -1 & -1 & 5.5 & 3 & 10 & 3.5 & 19 & 0 & 1 & 0 & 0 & 5 & 3 & 15 & 4 \\
\hline 7 & -1 & 1 & 1 & -1 & 4.5 & 3 & 20 & 3.5 & 20 & 0 & -1 & 0 & 0 & 5 & 2 & 15 & 4 \\
\hline 8 & -1 & -1 & 1 & 1 & 4.5 & 2 & 20 & 4.5 & 21 & 0 & 0 & 1 & 0 & 5 & 2.5 & 20 & 4 \\
\hline 9 & -1 & 1 & -1 & 1 & 4.5 & 3 & 10 & 4.5 & 22 & 0 & 0 & -1 & 0 & 5 & 2.5 & 15 & 4 \\
\hline 10 & 1 & -1 & 1 & -1 & 5.5 & 2 & 20 & 3.5 & 23 & 0 & 0 & 0 & 1 & 5 & 2.5 & 20 & 4.5 \\
\hline 11 & 1 & -1 & -1 & 1 & 3.5 & 2 & 10 & 4.5 & 24 & 0 & 0 & 0 & -1 & 5 & 2.5 & 20 & 3.5 \\
\hline 12 & 1 & 1 & 1 & -1 & 5.5 & 3 & 20 & 3.5 & 25 & 0 & 0 & 0 & 0 & 5 & 2 & 20 & 4 \\
\hline 13 & -1 & 1 & 1 & 1 & 4.5 & 3 & 20 & 4.5 & 26 & 0 & 0 & 0 & 0 & 5 & 2 & 20 & 4 \\
\hline
\end{tabular}

Table 3 Experiment Results for Fine Dolomitic Phosphate Pebble Column Flotation

\begin{tabular}{|c|c|c|c|c|c|c|c|c|c|c|c|c|c|c|}
\hline \multirow[t]{2}{*}{ Run } & \multicolumn{4}{|c|}{ Coded conditions } & \multicolumn{4}{|c|}{ Natural conditions } & \multirow{2}{*}{ Product } & \multirow{2}{*}{ Wt.\% } & \multicolumn{2}{|c|}{ Grade, \% } & \multicolumn{2}{|c|}{ Recovery, \% } \\
\hline & pH & CL & IF & AR & $\mathbf{p H}$ & $\mathbf{C L}$ & IF & AR & & & $\mathbf{P}_{2} \mathbf{O}_{5}$ & MgO & $\mathbf{P}_{2} \mathbf{O}_{5}$ & MgO \\
\hline \multirow{3}{*}{1} & \multirow{3}{*}{-1} & \multirow{3}{*}{-1} & \multirow{3}{*}{-1} & \multirow{3}{*}{-1} & \multirow{3}{*}{4.5} & \multirow{3}{*}{2} & \multirow{3}{*}{10} & \multirow{3}{*}{3.5} & Float & 3.40 & 18.80 & 4.15 & 2.48 & 10.24 \\
\hline & & & & & & & & & Sink & 96.60 & 26.06 & 1.28 & 97.52 & 89.76 \\
\hline & & & & & & & & & Feed & & 25.81 & 1.38 & 100.00 & 100.00 \\
\hline \multirow{3}{*}{2} & \multirow{3}{*}{1} & \multirow{3}{*}{-1} & \multirow{3}{*}{-1} & \multirow{3}{*}{-1} & \multirow{3}{*}{5.5} & \multirow{3}{*}{2} & \multirow{3}{*}{10} & \multirow{3}{*}{3.5} & Float & 5.20 & 20.15 & 4.74 & 3.94 & 18.38 \\
\hline & & & & & & & & & Sink & 95.20 & 26.86 & 1.15 & 96.06 & 81.62 \\
\hline & & & & & & & & & Feed & & 26.62 & 1.34 & 100.00 & 100.00 \\
\hline
\end{tabular}




\begin{tabular}{|c|c|c|c|c|c|c|c|c|c|c|c|c|c|c|}
\hline \multirow{3}{*}{3} & \multirow{3}{*}{-1} & \multirow{3}{*}{1} & \multirow{3}{*}{-1} & \multirow{3}{*}{-1} & \multirow{3}{*}{4.5} & \multirow{3}{*}{3} & \multirow{3}{*}{10} & \multirow{3}{*}{3.5} & Float & 7.33 & 20.68 & 3.77 & 5.90 & 20.69 \\
\hline & & & & & & & & & Sink & 91.31 & 26.50 & 1.16 & 94.10 & 79.31 \\
\hline & & & & & & & & & Feed & & 25.71 & 1.34 & 100.00 & 100.00 \\
\hline \multirow{3}{*}{4} & \multirow{3}{*}{-1} & \multirow{3}{*}{-1} & \multirow{3}{*}{1} & \multirow{3}{*}{-1} & \multirow{3}{*}{4.5} & \multirow{3}{*}{2} & \multirow{3}{*}{20} & \multirow{3}{*}{3.5} & Float & 3.80 & 20.30 & 4.93 & 2.98 & 13.39 \\
\hline & & & & & & & & & Sink & 96.20 & 26.08 & 1.26 & 97.02 & 86.61 \\
\hline & & & & & & & & & Feed & & 25.86 & 1.40 & 100.00 & 100.00 \\
\hline \multirow{3}{*}{5} & \multirow{3}{*}{-1} & \multirow{3}{*}{-1} & \multirow{3}{*}{-1} & \multirow{3}{*}{1} & \multirow{3}{*}{4.5} & \multirow{3}{*}{2} & \multirow{3}{*}{10} & \multirow{3}{*}{4.5} & Float & 5.39 & 19.46 & 4.04 & 3.97 & 15.76 \\
\hline & & & & & & & & & Sink & 94.61 & 26.84 & 1.23 & 96.03 & 84.24 \\
\hline & & & & & & & & & Feed & & 26.44 & 1.38 & 100.00 & 100.00 \\
\hline \multirow{3}{*}{6} & \multirow{3}{*}{1} & \multirow{3}{*}{1} & & & & & & & Float & & & & & \\
\hline & & & -1 & -1 & 5.5 & 3 & 10 & 3.5 & Sink & & & & & \\
\hline & & & & & & & & & Feed & & & & & \\
\hline & & & & & & & & & Float & 6.67 & 22.6 & 3.73 & 5.62 & 18.30 \\
\hline 7 & -1 & 1 & 1 & -1 & 4.5 & 3 & 20 & 3.5 & Sink & 93.33 & 27.10 & 1.19 & 94.38 & 81.70 \\
\hline & & & & & & & & & Feed & & 26.80 & 1.36 & 100.00 & 100.00 \\
\hline & & & & & & & & & Float & 5.12 & 17.5 & 4.4 & 3.48 & 17.48 \\
\hline 8 & -1 & -1 & 1 & 1 & 4.5 & 2 & 20 & 4.5 & Sink & 94.88 & 26.19 & 1.12 & 96.52 & 82.52 \\
\hline & & & & & & & & & Feed & & 25.75 & 1.29 & 100.00 & 100.00 \\
\hline & & & & & & & & & Float & 8.14 & 18.1 & 4.77 & 5.67 & 26.88 \\
\hline 9 & -1 & 1 & -1 & 1 & 4.5 & 3 & 10 & 4.5 & Sink & 91.86 & 26.70 & 1.15 & 94.33 & 73.12 \\
\hline & & & & & & & & & Feed & & 26.00 & 1.44 & 100.00 & 100.00 \\
\hline 10 & 1 & -1 & 1 & -1 & 55 & 2 & 20 & 35 & Float & 4.23 & 19.67 & 3.92 & 3.20 & 12.00 \\
\hline & & & & & & & & & Sink & 95.76 & 26.30 & 1.27 & 96.80 & 88.00 \\
\hline
\end{tabular}




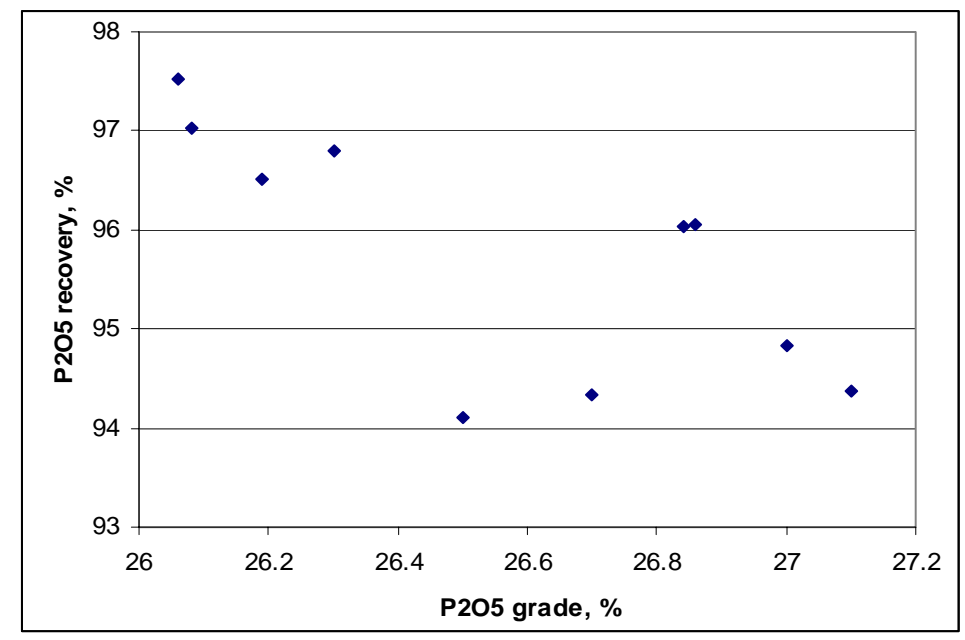

Figure 1 Relationship of $\mathrm{P}_{2} \mathrm{O}_{5}$ Recovery and $\mathrm{P}_{2} \mathrm{O}_{5}$ Grade

\section{SUMMARY}

Experiment runs using flotation column for fine dolomitic phosphate pebble flotation based on Center Composite Experiment Design have been conducted in systematic tests. The results show that the $\mathrm{P}_{2} \mathrm{O}_{5}$ recovery is very high (over 95\%), but $\mathrm{MgO}$ rejection is low due to possible insufficient of collector dosages for this period of the experiment design runs, which can be observed from the appearance and thickness of froth layers. The improvement in reduction of $\mathrm{MgO}$ is expecting for the rest of the tests for the experiment design runs which required more higher dosages.

\section{FUTURE WORK}

Experiment design runs for fine dolomitic phosphate pebble column flotation based on Center Composite Experiment Design for systematic testing will be continued in systematic approach, and to complete experiment design runs. The results will be subject to analyzed for ANOVA using JMP software tools for $\mathrm{P}_{2} \mathrm{O}_{5}$ recovery and $\mathrm{MgO}$ rejection as the responses.

\section{REFERENCES}

Hwang, C., Guan, C., Gao Z., Zheng, Z., and Xu, Q., Optimizing the Formulation for Dolomite Collector "PA-31" Using Raw Materials From the United States, Final Report No. 02-150-197, submitted to Florida Institute of Phosphate Research, Bartow, FL., April 2003.

Peng, F. F., and Gu, Z. X., 2003, "Separation of dolomite from phosphate using fatty acid soap collector by structure packing flotation column, Preprint 03-153, 2003 SME Annual Meeting, Cincinnati, OH., Feb. $24-26$.

\section{PUBLICATIONS/PRESENTATIONS}

None. 
Appendix G: Development of a Novel Fine Particle Centrifuge 


\section{TECHNICAL PROGRESS REPORT}

\begin{tabular}{llll} 
Contract Title and Number: & \multicolumn{2}{l}{ Period of Performance: } \\
Establishment of the Center for Advanced & Starting Date: & 9/17/01 \\
Separation Technologies (DE-FC26-01NT41091) & Ending Date: & 5/31/05 \\
\hline
\end{tabular}

Sub-Recipient Project Title:

Development of a Novel Fine Particle Centrifuge

Principal Investigators:

Roe-Hoan Yoon and Gerald H. Luttrell

Contact Address:

146 Holden Hall

Virginia Polytechnic Institute \& State University

Blacksburg, VA 24061

Subcontractor Address:

No subcontracts issued.

$\begin{array}{ll}\text { Report Information: } \\ \text { Type: } & \text { Semi-Annual } \\ \text { Number: } & 2 \\ \text { Period: } & 4 / 1 / 04-9 / 30 / 04 \\ \text { Date: } & 11 / 23 / 04 \\ \text { Code: } & \text { VA006 }\end{array}$

Contact Information:

Phone: (540) 231-4508

Fax: $\quad$ (540) 231-3948

E-Mail: cast@vt.edu

Subcontractor Information:

Phone:

Fax:

E-Mail:

\section{ABSTRACT}

The solid-solid separation processes employed by modern coal preparation plants require large amounts of process water. After cleaning, the unwanted water must be removed from the surfaces of the particles using mechanical dewatering equipment. Coarse particles can be readily dewatered using simple screening systems, while finer particles require more complicated unit operations such as centrifuges and filters. Unfortunately, the processes used to dewater fine particles are inherently inefficient and costly to operate and maintain. To overcome this problem, a novel hyperbaric centrifugal filter has been developed by researchers at Virginia Tech. Preliminary test data obtained using a batch unit suggest that this new technology can reduce the moisture content of fine coal products by approximately $30-50 \%$ as compared to existing dewatering processes. The objective of this project will be to construct a continuous prototype unit and to conduct a detailed experimental study of this new technology. Activities performed during this reporting period include (i) fabrication work required to construct a $100 \mathrm{lb} / \mathrm{hr}$ semi-continuous prototype and (ii) theoretical studies of the underlying mechanisms that occur during hyperbaric centrifugation. 


\section{INTRODUCTION}

\section{Background}

One of the most difficult and costly steps in coal preparation is the removal of moisture from the surfaces of fine coal particles. Although thermal dryers can effectively reduce moisture, these units require large capital expenditures and stringent air quality standards make it impossible to obtain new operating permits. As a result, coal producers resort to mechanical systems for fine coal dewatering. Unfortunately, the performance of mechanical dewatering equipment diminishes sharply for particles finer than $0.1 \mathrm{~mm}$. The unacceptably high moisture content associated with this fraction forces the majority of coal producers to discard their coal fines to waste impoundments. In the U.S. alone, approximately 2 billion tons of fine coal has been discarded in abandoned ponds and 500 to 800 million tons in active ponds. On a yearly basis, U.S. coal producers discard approximately 30 to 40 million tons of fresh coal fines into ponds. This represents a loss of valuable natural resources, loss of profit for coal producers, and creation of significant environmental concerns.

\section{Objective and Approach}

The availability of a low-cost mechanical dewatering device that can efficiently remove moisture from fine coal will greatly benefit the U.S. coal industry. In light of this need, researchers at Virginia Tech have developed a novel hyperbaric filter centrifuge that can reduce the moisture contents of fine coal by $30-50 \%$ as compared to currently available dewatering methods (Yoon and Asmatulu, 2000). The new technology employs both gas pressure and centrifugal force to increase the driving force for removing water from the fine capillaries present in a filter cake. The specific objectives of this project are (i) to construct a small-scale continuous prototype unit for testing, (ii) to evaluate the effects of key operating and design variables, and (iii) to demonstrate the capabilities of the new technology using fine coals from different coal preparation plants.

\section{PROJECT TASKS}

\section{$\underline{\text { Task } 1 \text { - Filter Centrifuge Design/Construction }}$}

The objective of this task is to design and construct a prototype of the hyperbaric filter centrifuge. As stated in the last technical progress report, many conceptual designs were assessed before one was chosen. The selected design consists of a two-stage system incorporating separate thickening and dewatering steps (Figure 1). The first stage, which consists of a thickening tank, efficiently separates water from feed solids at a high capacity. This step ensures that high solids recoveries are maintained. The second stage, which consists of a rotating pressure chamber lined with wedge-shaped bars $(0.1 \mathrm{~mm}$ openings), dewaters the thickened feed using a combination of gas pressure and centrifugal force (Figure 2). This particular step ensures that good product moistures are 
maintained. Fine solids that pass with the water through the wall of the pressure chamber are recycled back to the thickener.

The cake discharge system was considered to be the most critical element in the design. The cake is discharged using a pusher/scraper equipped with a Teflon pressure seal to minimize leakage (Figure 3). The seal is designed as a wear component that can be easily accessed and replaced. In addition, the spacing bars had to be fabricated with a very tight tolerance (i) to prevent the excessive loss of solids though the screen and (ii) to provide a good seal against the wall of the pressure chamber. The bars were obtained from a centrifuge manufacturer who obtains prefabricated parts pressed from tungsten carbide (Figure 4). This makes the bars highly wear resistant and ensures that the same screen opening is maintained.

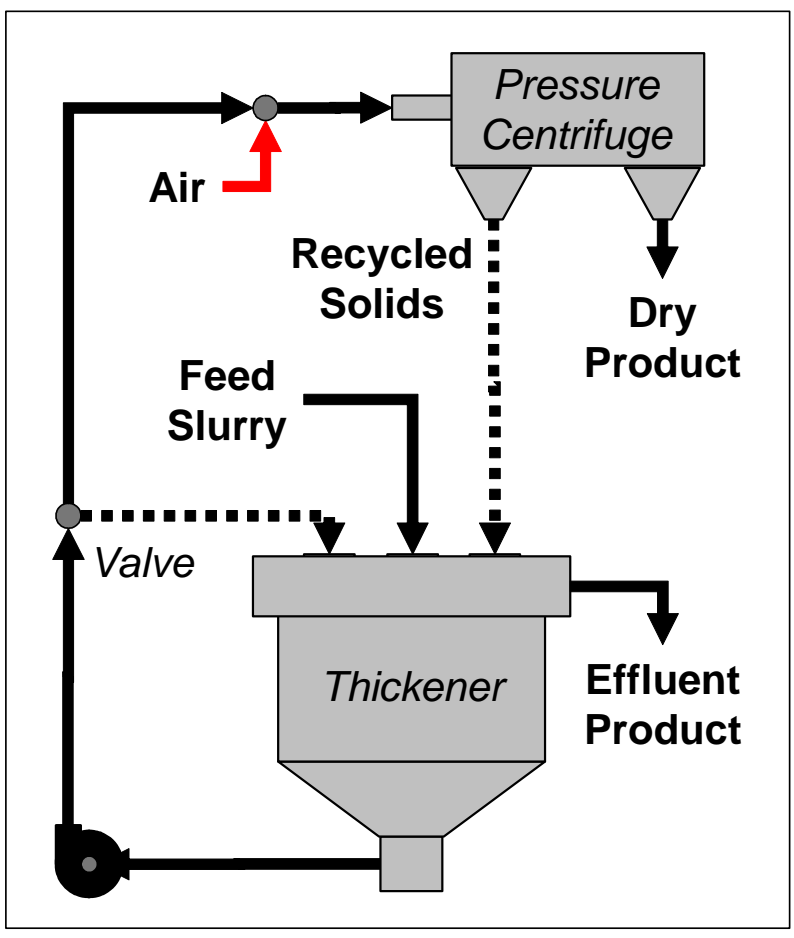

Figure 1. Hyperbaric filter centrifuge (cake moisture controlled by the centrifuge and solids recovery controlled by the thickener).

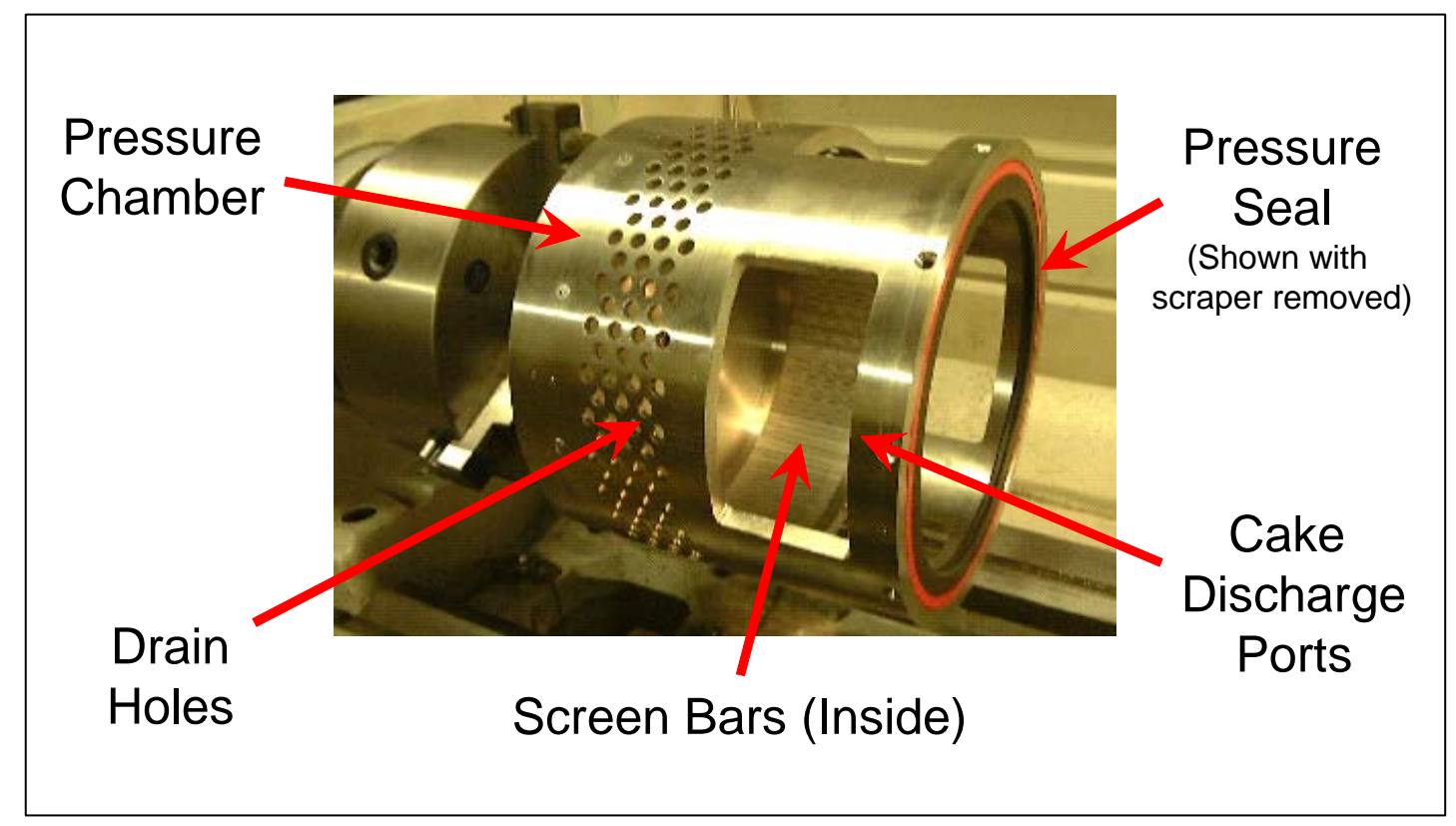

Figure 2. Key components of the rotating centrifuge pressure chamber. 


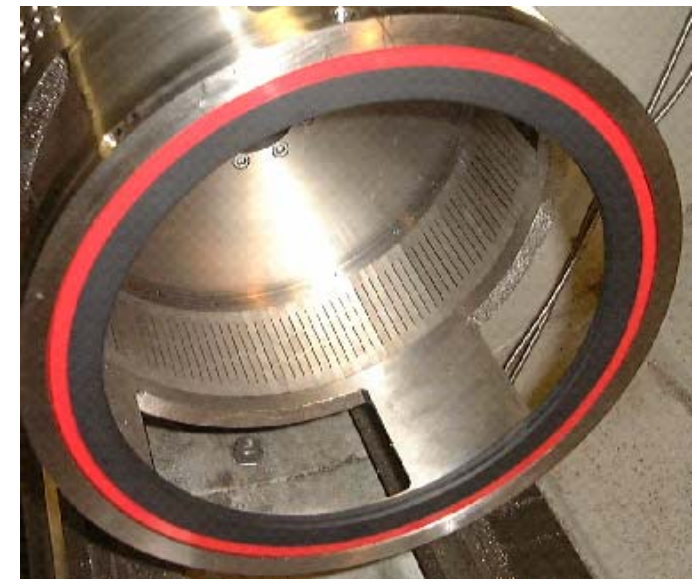

Figure 3. Photo of Teflon pressure seal (red) and tungsten carbide spacer bars (back). (Shown with scraper removed.)

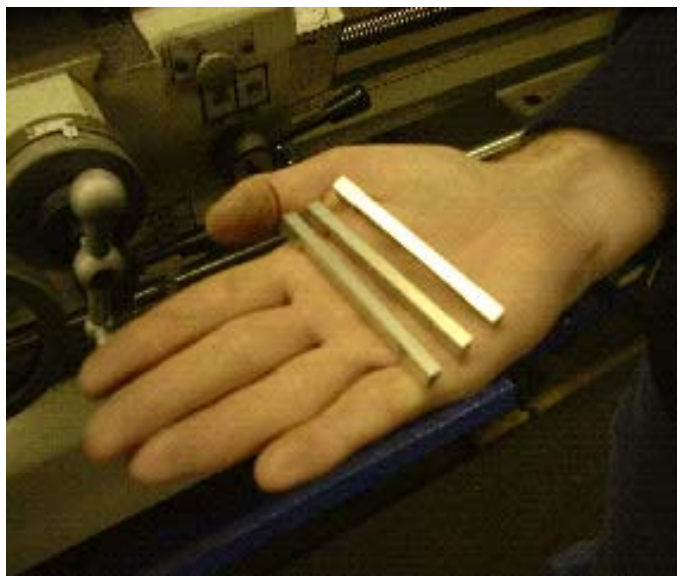

Figure 4. Photo showing the tungsten carbide spacer bars prior to installation inside the rotating pressure chamber.

\section{Task 2 - Filter Centrifuge Testing}

To date, no work has been done on this task pending the completion of the construction of the prototype unit.

\section{Task 3 - Engineering Design}

To date, no work has been done on this task pending the completion of the construction of the prototype unit.

\section{Task 4 - Process Modeling}

Batch tests conducted to date indicate that the cake moisture produced by a basket-type centrifugal filter can be substantially reduced via the introduction of compressed air into the filter chamber. During the past reporting period, a theoretical analysis was conducted to develop a better understanding of the underlying mechanisms of dewatering by hyperbaric centrifugation. To simplify this analysis, the model system has been assumed to consist on a bed of settled particles initially submerged in water (Figure 1). This system can be readily described by the radii between the rotational axis of the centrifuge and the water level $\left(\mathrm{r}_{\mathrm{w}}\right)$, cake surface $\left(\mathrm{r}_{\mathrm{s}}\right)$, and bowl wall $\left(\mathrm{r}_{\mathrm{b}}\right)$. The bed of particles is assumed to be comprised of a series of straight capillaries that radiate outward through the filter cake, an assumption that is commonly used in elementary studies of filtration phenomenon. The analysis also assumes that the resisting and driving forces that control the dewatering must be equal at the equilibrium moisture content. This 
assumption makes it possible to neglect hydrodynamic flow resistance since no flow occurs under equilibrium conditions. The influence of the filter medium on dewatering is also not considered in this elementary analysis.

In light of the stated assumptions, the primary force resisting the dewatering of the cake is the capillary pressure. In the case where $\mathrm{r}_{\mathrm{w}}<\mathrm{r}_{\mathrm{s}}$, the liquid level is above the cake surface and the capillary pressure $\left(\mathrm{P}_{\text {cap }}\right)$ is given by the well-known equation:

$$
\mathrm{P}_{\text {cap }}=\frac{2 \gamma \cos \theta}{\mathrm{r}_{\mathrm{c}}}
$$

where $\gamma$ is the interfacial surface tension, $\theta$ is the contact angle, and $r_{c}$ is the capillary radius. For $r_{\mathrm{w}}>\mathrm{r}_{\mathrm{s}}$, the cake is not fully saturated. This geometry introduces a second three phase contact that increases the resisting capillary pressure to a value given by:

$$
\mathrm{P}_{\text {cap }}=\frac{4 \gamma \cos \theta}{\mathrm{r}_{\mathrm{c}}}
$$

The equilibrium moisture can now be calculated when compressed gas is the only driving pressure. In this case, equilibrium requires that $\mathrm{P}_{\text {gas }}=\mathrm{P}_{\text {cap }}$, i.e.:

$$
\mathrm{P}_{\text {gas }}=\frac{4 \gamma \cos \theta}{\mathrm{r}_{\mathrm{c}}}
$$

After starting with a saturated cake (Figure 2a), this expression indicates that all water is forced from capillaries for which $\mathrm{P}_{\text {gas }}>\mathrm{P}_{\text {cap }}$. Water is removed entirely from all of the

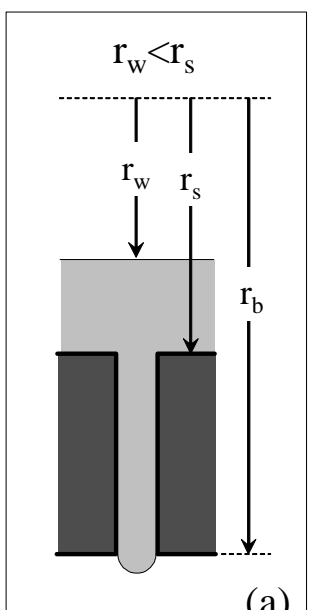

(a)

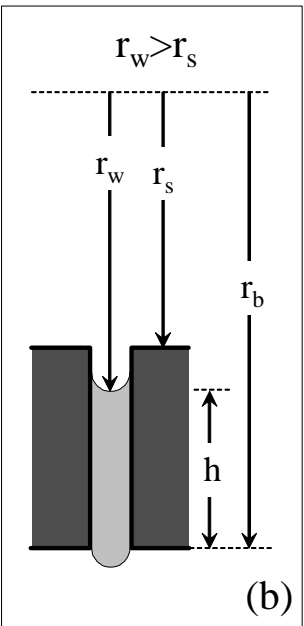

Figure 2. Geometry for capillary pressure when (a) $r_{w}<r_{s}$ and (b) $r_{w}>r_{s}$. (a) Saturated Cake

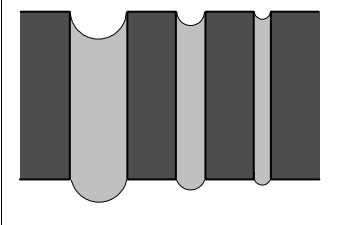

(c) Centrifugation Only

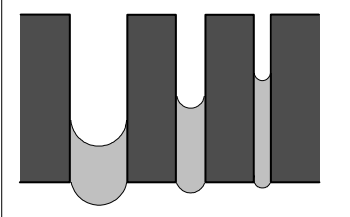

Figure 3. Response of various sizes of capillaries to different driving forces. 
larger capillaries until gas breakthrough occurs and gas pressure is lost. Unfortunately, water remains in the smaller capillaries for which $\mathrm{P}_{\text {gas }}<\mathrm{P}_{\text {cap }}$ (Figure $2 \mathrm{~b}$ ). For a specified gas pressure, there is no reduction in the height of liquid (h) in capillaries smaller than the critical capillary radius defined by Eq. [3]. As a result, not all of the water can be removed from the cake capillaries using gas pressure as the only driving force.

When the centrifugal field is the only driving force, then the equilibrium requires that $\mathrm{P}_{\text {hyd }}=\mathrm{P}_{\text {cap }}$. For any distance $\mathrm{r}$ from the rotational axis, the hydrostatic pressure differential $\left(\mathrm{P}_{\text {hyd }}\right)$ created by the centrifugal field can be calculated by integration of:

$$
\mathrm{P}_{\text {hyd }}=\rho \int_{\mathrm{r}_{\mathrm{w}}}^{\mathrm{r}_{\mathrm{b}}} \mathrm{r} \omega^{2} \mathrm{dr}=\frac{\rho \omega^{2} \mathrm{r}_{\mathrm{b}}^{2}}{2}\left[1-\left(\frac{\mathrm{r}_{\mathrm{w}}}{\mathrm{r}_{\mathrm{b}}}\right)^{2}\right]
$$

where $\rho$ is the fluid density and $\omega$ is the angular velocity, Since the height of water in the capillary (h) is equal to $\mathrm{r}_{\mathrm{b}}-\mathrm{r}_{\mathrm{w}}$ and $\mathrm{h} / \mathrm{r}_{\mathrm{b}}<<1$, Eq. [4] can be simplified to:

$$
\mathrm{P}_{\text {hyd }}=\frac{\rho \omega^{2} \mathrm{r}_{\mathrm{b}}^{2}}{2}\left[1-\left(1-2 \frac{\mathrm{r}_{\mathrm{w}}}{\mathrm{r}_{\mathrm{b}}}\right)\right]=\rho \omega^{2} \mathrm{r}_{\mathrm{b}} \mathrm{h}
$$

By combining Eqs. [2] and [5], we obtain:

$$
\mathrm{h}=\frac{4 \gamma \cos \theta}{\rho \omega^{2} \mathrm{r}_{\mathrm{b}} \mathrm{r}_{\mathrm{c}}}
$$

This expression shows that the height of water in capillaries of all different sizes is reduced proportionally in accordance with capillary radius (Figure 2c). This response is in sharp contrast to the case in which only gas pressure is used and water is removed only from capillaries for which $\mathrm{P}_{\text {gas }}>\mathrm{P}_{\text {cap. }}$. On the other hand, the driving force for additional water removal by centrifugation disappears for all capillaries when the water height (h) reaches the value dictated by Eq. [6]. As a result, not all of the water can be removed from the capillaries using centrifugation as the only driving force.

Now consider the case in which both gas pressure and centrifugation are combined for improved dewatering. Equilibrium for this case requires that $\mathrm{P}_{\text {hyd }}+\mathrm{P}_{\text {gas }}=$ $\mathrm{P}_{\text {cap }}$, which dictates that:

$$
\mathrm{h}=\frac{1}{\rho \omega^{2} \mathrm{r}_{\mathrm{b}}}\left[\frac{4 \gamma \cos \theta}{\mathrm{r}_{\mathrm{c}}}-\mathrm{P}_{\text {gas }}\right]
$$

In this case, the height of water in all of the capillaries predicted by Eq. [6] is further reduced by the gas pressure $\left(\mathrm{P}_{\mathrm{gas}}\right)$. More importantly, the gas pressure removes water completely from capillaries for which $\mathrm{P}_{\text {gas }}>\mathrm{P}_{\text {cap }}$ (Figure 2d). As stated previously, this is not possible using centrifugation alone once the volume of water in the capillary is so 
small that $\mathrm{P}_{\text {hyd }}<\mathrm{P}_{\text {cap }}$. Likewise, the centrifugal force removes water from smaller capillaries that cannot be removed by gas pressure alone. Therefore, the combined influence of gas pressure and centrifugal force provide a synergistic effect for cake dewatering.

\section{SUMMARY}

A project has been initiated to construct and test a hyperbaric filter centrifuge that is capable of substantially improving the removal of moisture from fine particle streams. Laboratory experiments performed using a batch laboratory unit indicates that moisture reductions of up to 50\% can be achieved using this new technology. During this reporting period, work was continued to complete the construction of a continuous prototype. The prototype system is comprised of a two-stage process incorporating a thickening step and dewatering step. Once construction is completed, the prototype unit will be used (i) to demonstrate the capabilities of this new technology at a larger scale and (ii) to obtain scale-up data for the engineering design of a production scale unit.

\section{FUTURE WORK}

The next step in this project is to complete fabrication of the hyperbaric centrifuge and begin testing as described under Task 2. Also, laboratory-scale tests will run concurrently using the lab centrifuge to further understand and optimize each operating parameter in this novel centrifugation process.

\section{REFERENCES}

Yoon, R.-H. and R. Asmatulu, "Methods of Improving Centrifugal Filtration,” U.S. Patent Application Serial No. 09/531,373, filed March 21, 2000.

Yoon R.-H. and R. Asmatulu, PCT Patent Application Serial No. PCT/US02/11318, filed April 12, 2002.

\section{PUBLICATIONS/PRESENTATIONS}

To date, no major publications have resulted from this project. 
Appendix H: New Strategies for Dewatering of Coals 


\section{TECHNICAL PROGRESS REPORT}

Contract Title and Number: Crosscutting Technology Development at the Center for Advanced Separation Technologies (DE-FC26-02NT41607)

\section{Sub-Recipient Project Title:}

New Strategies for dewatering of coals

Principal Investigators: M. S. Seehra

Co-investigators:

Contact Address:

Physics Department

West Virginia University

Morgantown, WV 26506-6315

Subcontractor Address:

No subcontracts issued.
Period of Performance:

Starting Date: 3/03

Ending Date: 5/31/05
Report Information:

Type: Semi-Annual

Number: 03

Period: $\quad 3 / 04$ to $9 / 04$

Date: $\quad 9 / 27 / 04$

Code: WV004-R003

Contact Information:

Phone: $\quad 304-293-3422$ x1473

Fax: $\quad 304-293-5732$

E-Mail: mseehra@wvu.edu

Subcontractor Information:

Phone:

Fax:

E-Mail:

\section{ABSTRACT}

During this reporting period, our efforts were directed at designing and constructing a laboratory-scale coal dewatering apparatus using the conventional $2.45 \mathrm{GHz}$ microwaves. A commercial microwave oven has been modified so that wet coal can be fed to a microwave oven on a conveyor belt. This open design necessitated substantial microwave shielding and a remote control electronic circuit. Details of the systems are presented in this report, along with some preliminary results. Also included is a description of the status of the modeling studies of coal-water interactions.

\section{INTRODUCTION}

Since water is used extensively in coal preparation and cleaning, dewatering of coals is required prior to shipment to power plants. However, finer fractions of coals are difficult to dewater and consequently these fractions are relegated to settling ponds, causing environmental problems and loss of a large fraction of coals. Thermal drying of coals is not an efficient process since hot air heats both coal and water. An objective of this project is to investigate new strategies for coal dewatering which involve selective energy absorption by 
water without heating coal. These new strategies include selective heating by microwaves and infrared radiation combined with modeling of the coal-water interactions.

\section{PROJECT TASKS}

In the previous report, we presented results on gram quantities of a wet coal showing that dewatering of coals by resonant microwave drying is a very efficient process in that drying efficiency near the theoretical maximum of $1.4 \mathrm{~kg}$ of water per kilowatt-hour of power used is obtainable without significantly heating the coals. Since then, we have designed a laboratory-scale microwave dewatering unit with a conveyor-belt feed system to simulate a potential commercial unit. This unit was designed with the assistance of Phil Tucker, the electronic technician in the Physics Department and built with the assistance of personnel in our machine shop. The graduate assistant A. Kalra and co-investigator A. Manivannan were the lead personnel for this effort with oversight and guidance provided by the Principal Investigator. The modeling work was carried out by the co-investigator, M. Bachlechner. This report was prepared by the PI using input from all the investigators of the project.

(a) Laboratory-Scale Microwave Dewatering Unit: A schematic diagram of this unit is shown in Fig. 1. It consists of a store-bought 850 watt microwave oven whose two opposite sides were opened up for feeding a conveyor-belt. The conveyor-belt is fed by two motor driven rollers mounted at the end of the table. The feed-speed is controlled by the rotation speed of the rollers. Because of the open microwave oven, extensive shielding from microwaves is needed. Since microwaves are absorbed by any good electrical conductor, we have used store-bought wire-mesh for shielding, double layer of which surrounded the whole apparatus (shielding not shown in Fig. 1). A portable microwave detector was used to check for any leaks of the microwaves outside the apparatus. Since the operator of the system must be protected from any microwaves, an electrical circuit was designed to control the operation of the system from a distance. A basic design of this electrical system for the microwave unit is shown in Fig. 2 and for the control system in Fig. 3. The main consideration in this design is that with the remote control switch, power to the microwave oven and the conveyor-belt motor can be controlled. We are looking into acquiring a better shielding system (perhaps leaded-glass which is often used for shielding x-rays) which will not only provide necessary shielding but also allow visual view of the system during operations.

Although the system has been tested successfully for operation in that significant dewatering of the coal was obtained after a single cycle, systematics studies have yet to be carried out. The factors that need to be investigated include: (i) determining the optimum resident time of the coal inside the oven which in turn will be controlled by the speed of the conveyor-belt; (ii) the amount of dewatering achieved in a given cycle by measuring the moisture content of the coal before and after microwave exposure; (iii) Any effects of microwaves on the impurities and the carbon content of the coal; and (iv) analysis of the feasibility of scaling up the process for a commercial demonstration unit. These issues will be addressed in coming months. 


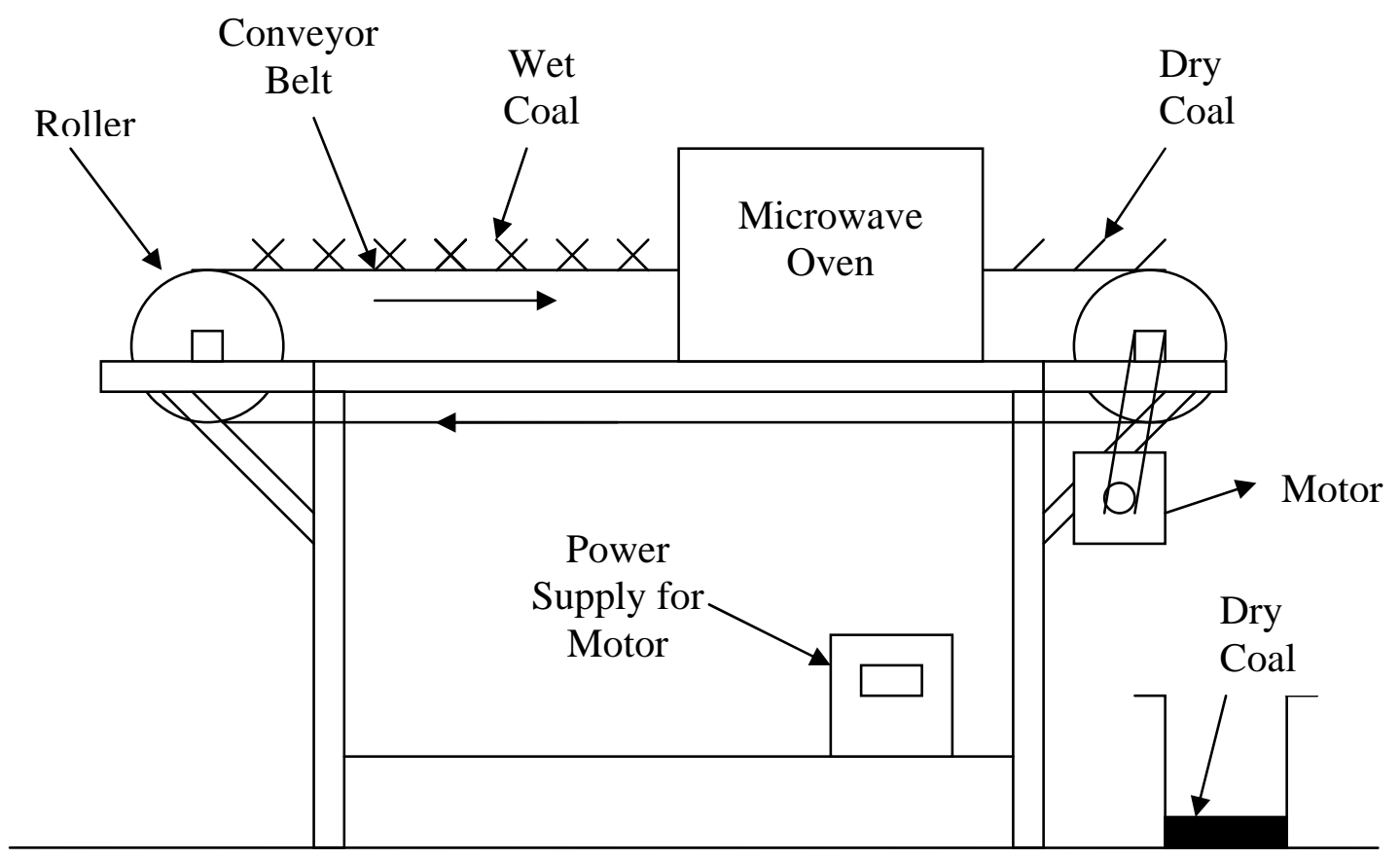

Figure (1) - Microwave Drying Setup

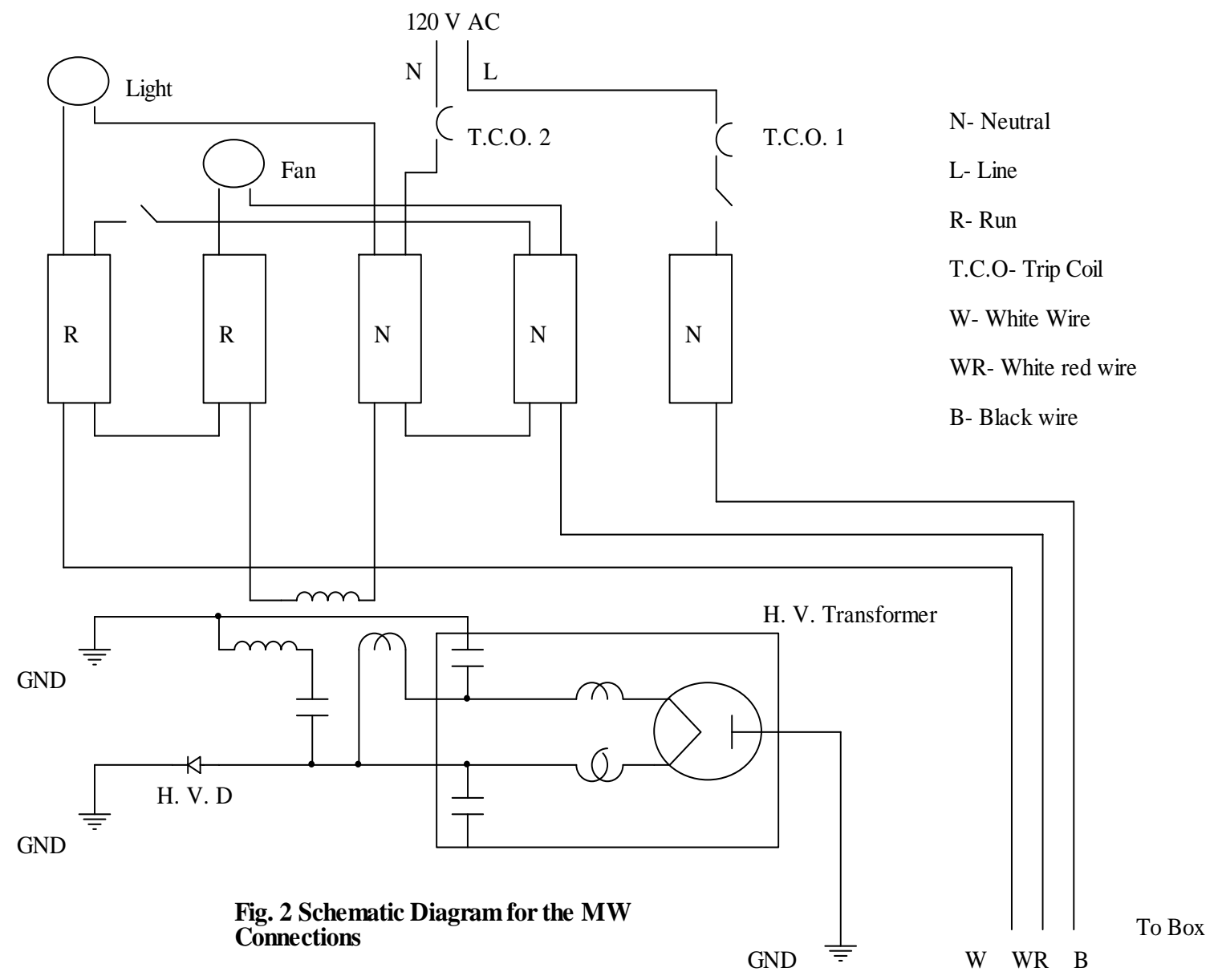




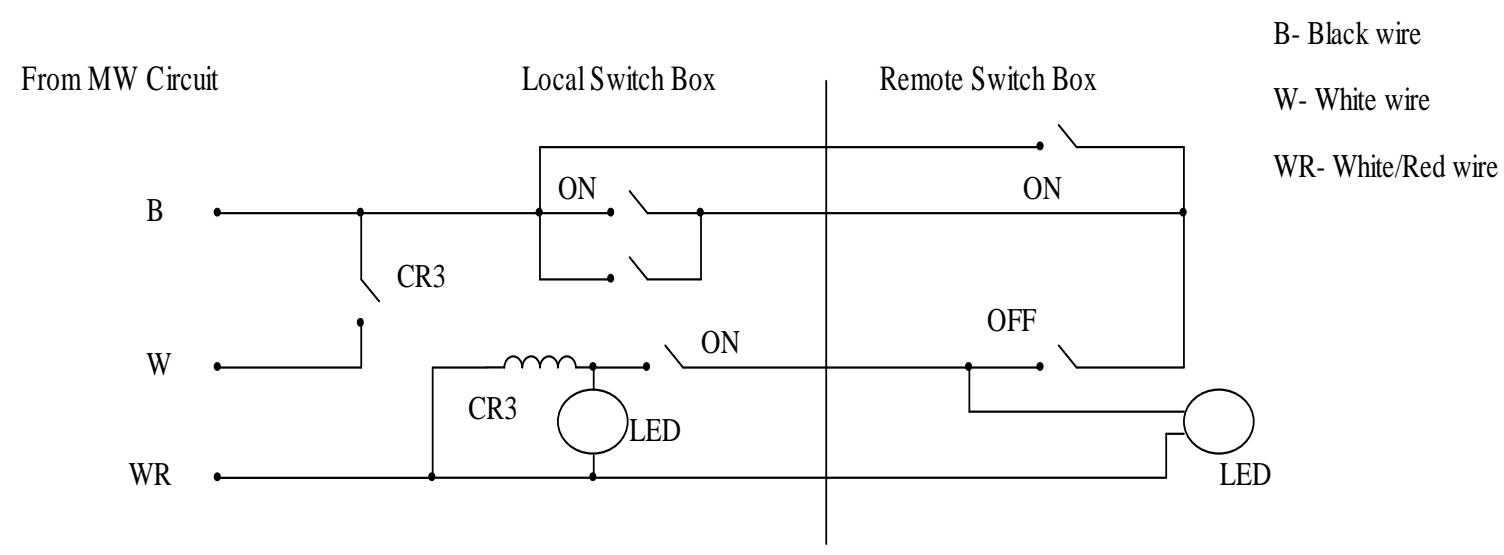

Fig 3. Schematic for Local and Remote Control

(b) Modeling the coal-water interface Dr. Bachlechner, a co-investigator on the project, is carrying out theoretical modeling studies of how water interacts with coal surface. This modeling work is based on the studies of Walther et al [1] who have modeled density profile of water around a carbon nanotube. Our studies are investigating this model's applicability to water on the surface of amorphous carbon simulating coal. The modeling studies involve the following types of pair interactions: (i) $\mathrm{O}-\mathrm{H}$ interaction within each $\mathrm{H}_{2} \mathrm{O}$ molecule; (ii) $\mathrm{O}-\mathrm{O}, \mathrm{H}-\mathrm{H}$ and $\mathrm{O}-\mathrm{H}$ interactions between different $\mathrm{H}_{2} \mathrm{O}$ molecules; (iii) C-O interaction between carbon and oxygen; and (iv) three-body interactions within a $\mathrm{H}_{2} \mathrm{O}$ molecule to ensure $109.47^{\circ}$ bond angle for $\mathrm{H}-\mathrm{O}-\mathrm{H}$. In order to determine the orientation of the water molecules at the carbon surface (and hence the water-coal interaction), we need to include quadrupole interaction between the carbon atoms and the partial charges on $\mathrm{H}$ and $\mathrm{O}$ atoms of $\mathrm{H}_{2} \mathrm{O}$. The computer code for this phase of the work is now under development.

[1]. J. H. Walther, R. Jaffe. T. Halicioglu, and P. Koumoutsakos, J. Phys. Chem. B $\underline{105}$, 9980 (2001).

\section{FUTURE WORK}

As outlined above at the end of section (a) under Project Tasks, systematic studies of the efficiency of the microwave dewatering using the laboratory scale unit (Fig. 1 and Fig. 2) are planned for the coming months. The belt speed and hence the optimum resident time of coal in the microwave unit, for maximum dewatering, needs to be determined. The modeling studies should provide some insight into the mechanism of water absorption in coals. 
Appendix I: Novel Bioleaching Technology Assisted by Electrolytic

\section{Processes}


TECHNICAL PROGRESS REPORT

Contract Title and Number:

Period of Performance:

Establishment of the Center for Advanced Separation

Starting Date: 9/17/2001

Technologies (DE-FC26-01NT41091)

Ending Date: 7/31/2005

Sub-Recipient Project Title:

Bioleaching Assisted by Electrolytic Processes

Principal Investigators: Eung Ha Cho

Co-investigators: Ray R-K Yang

Contact Address:

Department of Chemical Engineering

West Virginia University

P. O. Box 6102

Morgantown, WV 26506-6102
Report Information:

Type: Semi-Annual

Number: 05

Period: $\quad 4 / 1 / 04$ to $9 / 31 / 04$

Date: $\quad$ 9/31/04

Code: WV005-R005

Contact Information:

Phone: $\quad 304-293-2111$ x -2433

Fax: $\quad$ 304-293- 4139

E-Mail: Eung.Cho@mail.wvu.edu

Subcontractor Information:

Phone:

Fax:

E-Mail:

\section{ABSTRACT}

This project is based on the electro-bioleaching of sulfide minerals of pyrite $\left(\mathrm{FeS}_{2}\right)$, chalcopyrite $\left(\mathrm{CuFeS}_{2}\right)$ and sphalerite $(\mathrm{ZnS})$. The approach is focused on the growth of the bacteria $A$. ferrooxidans under the influence of applied direct current. The major objective is to optimize the conditions of the electro-bioleaching process.

During the period for this semi-annual report, the leaching of chalcopyrite was investigated in two different leaching modes. One was the bacteria leaching (bioleaching) and the other was the electro-bioleaching where solution is circulated from the bioreactor to the cathode compartment of an electrochemical cell and then from the cathode to the bioreactor back to produce and provide the bacteria nutrient, ferrous ion. It was found that the leaching conversion of the mineral in the former mode increased as time went on, reached $0.57 \%$ around 22 days and then tended to level off. However, the leaching 
conversion of the mineral in the latter mode reached only $0.45 \%$ at the same time period of 22 days but increased without leveling off. It reached $0.64 \%$ in 38 days. These results show that the effect of electro-bioleaching is not clearly pronounced. These effects will be investigated on pyrite and sphalerite in the next period.

\section{INTRODUCTION}

\section{Background}

It is known that the bacteria A. ferrooxidans catalyzes the leaching of sulfide minerals including sphalerite $(\mathrm{ZnS})$, chalcopyrire $\left(\mathrm{CuFeS}_{2}\right)$ and pyrite $\left(\mathrm{FeS}_{2}\right)$. The bacteria grow in their nutrient of ferrous ion and produce ferric ion. Thus if we reduce the ferric ion which is produced from the growth of the bacteria to ferrous ion, the bacteria will grow continuously and thus can catalyze the leaching of sulfide minerals. This is the idea of this project.

An experimental system is designed with two reactors. One is a bioreactor in which sulfide minerals are leached with the bacteria A. ferrooxidans and the other is an electrochemical cell in which a leaching byproduct, ferric ion, is reduced to ferrous ion at the cathode compartment. The solution will be recycled from the cathode compartment of the electrochemical cell to the bioreactor by a pump, and at the same time, the solution will be recycled back to the cathode compartment of the electrochemical cell from the bioreactor by gravitational force. In this way, the bacteria will grow continuously in the bioreactor and will catalyze the leaching reactions of sulfide minerals.

\section{Objective}

The major objective of this project is to optimize the conditions of the electro-bioleaching process.

\section{PROJECT TASKS}

This project was conducted in the following tasks:

\section{Task 4 - Bioleaching of Mineral (Chalcopyrite)}

The leaching of chalcopyrite was investigated with the bacteria cultured for 6 days at the initial solution $\mathrm{pH}$ of 1.5. The bioreactor had one-liter solution which consisted of $250 \mathrm{ml}$ of sub-cultured solution and $750 \mathrm{ml}$ of ferrous solution at 0.0719 molar. The results show that the leaching conversion of the mineral increased as time went on, reached $0.57 \%$ at 22 days and tended to level off afterward. It was observed that the total soluble iron concentration in the solution decreased as time went on probably by precipitation as jarosite (sodium ferric sulfate/hydroxide minerals). It was estimated to be around $30 \%$ in 38 days. This may be the reason that the leaching of the mineral leveled off after 22 days.

\section{Task 5 - Electro-bioleaching of Mineral (Chalcopyrite)}

The leaching of chalcopyrite was investigated in an apparatus combining a bioreactor and an electro-chemical cell. The solution was discharged from the bioreactor to the cathode compartment by gravitational force, ferric ion was reduced at the cathode compartment 
electrochemically, and the solution was pumped back to the bioreactor. An identical bioreactor and identical conditions with those of Task 4 were used. The cathode compartment of the cell was filled with one-liter of 0.05 - molar ferric solution and the anode compartment was filled with one-liter of 0.2-molar sodium sulfate solution. The catholyte was ferrous ion in the previous experiments but it was changed to ferric ion because the ferric ion might simulate the actual conditions in the field better. Also, the current was kept around $50 \mathrm{~mA}$ for 5 hours in a day. This current flow reduced the ferric ion to ferrous ion by $15 \%$ in a day. The results show that the leaching conversion of the mineral increased as time went on, reached $0.45 \%$ at 22 days and continuously increased to $0.64 \%$ in 38 days without leveling off. This is slightly better than that with bioleaching only; however, the effect of electro-bioleaching is not clearly pronounced. It was estimated that the loss of total soluble iron due to precipitation is less than $5 \%$ during the 38-day period which is much lower than that with the bioleaching only. This may be the reason that the leaching conversion of chalcopyrite in this electro-bioleaching increases continuously without leveling off.

Towards the end of this period, an analytical method for ferrous ion was adopted and used. This method is involved in titrating ferrous ion with dichromate using an indicator of barium diphenylamine sulfonate. Also, total soluble iron concentration was determined by reduction of ferric to ferrous with stannous chloride and then titration of ferrous to ferric using dichromate as in the other method. The old analytical methods for ferric (iodometric titration) and for total iron (AA) were replaced with these new methods.

\section{SUMMARY}

The leaching conversion of chalcopyrite sample in bioleaching increased as time went on, reached $0.57 \%$ at 22 days and then tended to level off. However, the leaching conversion of the mineral in the electro-bioleaching reached only $0.45 \%$ at the same time period of 22 days but increased without leveling off. It reached $0.64 \%$ in 38 days. These results show that the electro-bioleaching is slightly better than the bioleaching; however, the effect of the electro-bioleaching is not clearly pronounced.

\section{FUTURE WORK}

Future experimental work will be focused on the leaching of pyrite and sphalerite in the two leaching modes of bioleaching and electro-bioleaching.

\section{PUBLICATIONS/PRESENTATIONS}

No publication or presentation has been made from the work of this research project. 
Appendix J: Evaluation of Coal Cleaning Efficiency Using TransponderBased Density Tracers 


\section{TECHNICAL PROGRESS REPORT}

\begin{tabular}{|c|c|}
\hline Contract Title and Number: & Period of Performance: \\
\hline 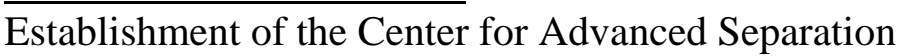 & $\overline{\text { Starting Date: } \quad 9 / 17 / 01}$ \\
\hline Technologies (DE-FC26-01NT41091) & Ending Date: \\
\hline
\end{tabular}

Sub-Recipient Project Title:

Evaluation Coal Cleaning Efficiency Using

Transponder-Based Density Tracers

Principal Investigators:

Gerald Luttrell and Roe-Hoan Yoon

Contact Address:

146 Holden Hall

Virginia Polytechnic Institute \& State University

Blacksburg, VA

Subcontractor Address:

No subcontracts issued.
Report Information:

Type: Semi-Annual

Number: 3

Period: $\quad$ 10/1/03-9/30/04

Date: $\quad 10 / 30 / 04$

Code: VA005

Contact Information:

Phone: (540)231-4508

Fax: (540)231-3948

E-Mail: cast@vt.edu

Subcontractor Information:

Phone:

Fax:

E-Mail:

\section{ABSTRACT}

Density tracers are one of the most powerful diagnostic tools for evaluating the performance of coal preparation plants. The objective of this project is to develop a new generation of density tracers that can be automatically tracked using RFID (radio frequency identification) technology. During previous reporting periods, controlled experiments were performed with static (stationary) transponders to evaluate the response of the RFID detection system to variables such as tracer-antenna orientation, detection distance, signal interference, reliability, etc. During this reporting period, dynamic tests were conducted using tracers in motion to examine variables such as charge up time, antenna placement, signal duplication/overlap, etc. Based on promising results obtained from these tests, a commercial prototype has been designed using off-the-shelf components for use in full-scale plant trials. Work is now focused on the manufacture of a large quantity (several hundred) of transponder-based tracers and the assembly of hardened reader-antenna system that can operate successfully in a harsh industrial environment. 


\section{INTRODUCTION}

\section{Background}

Density traces are plastic blocks (usually cubic) that incorporate high-density fillers to create artificial particles with known densities in the range of 1.20-2.60 SG. Density tracers offer a rapid and low-cost method for identifying problems with density-based processes that currently serve as the workhorse for the coal preparation industry. A typical plant test requires about an hour to complete as compared to several days (or even weeks) for traditional sampling and laboratory analysis. In addition, the tracer procedure is less costly and less hazardous to the environment compared to competitive float-sink tests that utilize toxic organic liquids. Unfortunately, the use of density tracers in industrial operations has been limited due to problems that occur during the retrieval step. The amount of labor required to manually collect the blocks can be excessive for large plants or complicated circuits. In addition, the data can sometimes be unreliable since tracers can be easily lost when buried in a thick bed of particles.

\section{Objective and Approach}

The objective of this project is to develop a new type of density tracer that can be automatically tracked using an electronic tagging system. The proposed system makes use of miniature tags (transponders) that are embedded within the tracer blocks when they are manufactured. These "passive" tags remain inactive until they become externally powered by a transmission signal from an antenna of a remote interrogator/reader. Once energized, the tags transmit a unique digital code back to the interrogator/reader that identifies the density and size of tracers reporting to either the clean coal or refuse products. The successful development of this technology will eliminate statistical counting errors associated with lost tracers and will make it possible for tests to be performed by a single individual. During the past reporting period, a Texas Instruments RFID system was tested to see if this low-cost, off-the-shelf technology could meet the strict requirements of the density tracer application.

\section{PROJECT TASKS}

\section{$\underline{\text { Task } 1 \text { - Transponder Evaluation }}$}

Several series of experiments were performed during the past reporting period to evaluate the capabilities of an off-the-shelf RFID detection system manufactured by Texas Instruments. Laboratory tests conducted using static (stationary) tracers indicated that this low-cost system could reliability detect tags embedded in density tracers, but some severe limitations existed in terms of effective read range (maximum allowable distance between the antenna and transponder tag). For example, a convenient location for the placement of the reader antenna would be on the clean coal belt and the refuse belt. In order for this location to be a viable option, a read range that is at least as deep as the load on the belt $(45-60 \mathrm{~cm})$ is required. While many factors influence the read range, the most important consideration is the power of the interrogation system. Because an RFID transponder is activated passively, it draws all of its power from the magnetic field created by the interrogation antenna. The 
available voltage falls off as $1 /$ distance $^{3}$ and the power falls off as $1 /$ distance $^{6}$. Other factors influencing the power requirement include the tag power demand, tag quality factor $(\mathrm{Q})$, tag tuning, tag antenna aperture, and reader antenna aperture.

The magnetic field used for activation of the transponder is related to the type of antenna(s) used to transfer power to the transponder and transmit data back to the reader. The size of the antenna determines how far the magnetic flux lines are emitted from the reader antenna. The larger the antenna the further the flux lines will extend into the region being monitored. The read range for a low frequency, inductively coupled, passive system is approximately one to two times the reader's antenna diameter. Larger antennas will yield a larger read area, but caution must be taken because the increased reception may lead to a worse signal-to-noise ratio at the receiver. The antenna needs to be tuned to resonate at the operating frequency in order for the maximum energy to be transferred to the transponder. The resonant frequency $\left(\mathrm{f}_{\mathrm{r}}\right)$, in a LC circuit, is described by:

$$
f_{r}=\frac{1}{2 \pi \sqrt{L C}}
$$

in which $\mathrm{L}$ is the inductance and $\mathrm{C}$ is the capacitance. The TI-RIS reader requires an antenna with an inductance of 26 to $27.9 \mu \mathrm{H}$. The antenna used in this system has an inductance of $24.4 \mu \mathrm{H}$. In order to bring the inductance up to $27 \mu \mathrm{H}$, a $0.0047 \mu \mathrm{F}$ capacitor is put in parallel with the antenna. In addition to being tuned to the resonant frequency, the antenna should have a $\mathrm{Q}$ factor of 100. ( $\mathrm{Q}$ is defined as the ratio of the effective impedance caused by the inductance of the coil at the frequency of transmission to the resistance in the antenna wire). The $\mathrm{Q}$ factor can be calculated using:

$$
Q=\frac{2 \pi f_{r} L}{R}
$$

in which $\mathrm{R}$ is the resistance. Since Q effectively represents the ratio of stored energy to dissipated energy, a higher $\mathrm{Q}$ indicates that a larger amount of energy can be extracted from the reader's field. In the current configuration, $\mathrm{L}=27 \mu \mathrm{H}, \mathrm{f}_{\mathrm{r}}=134 \mathrm{kHz}$, and $\mathrm{R}=0.22$. This combination provides an acceptable $\mathrm{Q}$ factor of 105.

For this application, smaller $(23 \mathrm{~mm})$ transponders are needed so that they can be embedded in density tracers that have sizes similar to the coal being treated. A ferrite rod can be used to focus the magnetic flux lines into the transponder's antenna. This is common in applications where the aperture of the antenna is less than $1 \mathrm{~cm}$. Another factor that can affect the read range is noise. Depending on the type of signal modulation that is employed the signal can be corrupted by electronic noise in the environment. The FSK (frequency shift keying) employed by the TI transponder is better than ASK (amplitude shift keying) at being immune to surrounding noise. In addition to the system design, there are also several environmental factors that affect the read range. The orientation of the transponder, movement of the transponder, and metal in the proximity are examples of application specific factors that also affect the read range. 


\section{$\underline{\text { Task } 2 \text { - Transponder Procurement }}$}

As indicated in the last technical report, a prototype reader system, field antenna, and associated transponder tags were procured for use in this project. Steps are now underway to procure an assortment of additional tags for manufacturing tracer blocks of various SG values for field testing in an industrial preparation plant.

\section{Task 3 - Preparation of Density Tracers}

During the past reporting period, steps were undertaken to initiate the manufacture of prototype density tracers for this project. Density tracers are normally cast by mixing a highdensity filler (such as zinc oxide) with a low-density resin (such as epoxy). A small transponder tag with a unique digital code is placed inside each block during casting. A colored pigment or dye is also added to make the block easier to see by eye during manual collection. A proprietary manufacturing procedure is used to ensure that the filler and pigment is homogeneously distributed and the mixture is free of entrained air bubbles.

The manufacturing of density tracers with an acceptable density tolerance was one of the major challenges that had to be addressed in this project. Ideally, the electronic tracers should have a density tolerance of at least \pm 0.01 SG. The presence of the glass-encased transponder complicates the manufacturing process since the block is no longer homogeneous (i.e., the mass and volume of the transponder must also be considered). Mathematically, the composite density of the tracer block $(\rho)$ can be calculated using:

$$
\rho=\rho_{\mathrm{t}} \phi_{\mathrm{t}}+\frac{1-\phi_{\mathrm{t}}}{\left(\frac{1-\mathrm{m}_{\mathrm{f}}}{\rho_{\mathrm{r}}}+\frac{\mathrm{m}_{\mathrm{f}}}{\rho_{\mathrm{f}}}\right)}
$$

in which $\mathrm{m}_{\mathrm{f}}$ is the mass fraction of high-density filler, $\phi_{\mathrm{t}}$ is the volume fraction occupied by the transponder tag, and $\rho_{\mathrm{t}}, \rho_{\mathrm{r}}$, and $\rho_{\mathrm{f}}$ are the densities of the transponder tag, dried resin, and filler, respectively. Using this relationship as a guide, a set of test blocks was prepared to evaluate the precision of the manufacturing procedures. The densities of the blocks were experimentally measured using a density balance, i.e.:

$$
S G=\frac{W_{A}}{W_{A}-W_{W}}
$$

in which SG is the specific gravity of the block, $\mathrm{W}_{\mathrm{A}}$ is the block weight when suspended in air, and $\mathrm{W}_{\mathrm{W}}$ is the block weight when suspended in water. The results obtained from these measurements are summarized in Table 1. All of the test blocks achieved the required tolerance specification of less than $\pm 0.01 \mathrm{SG}$, and all but one achieved a tolerance comparable to that typically obtained for conventional density tracers (i.e., $\pm 0.005 \mathrm{SG}$ ). 
Table 1. Measured SG Values for Manufactured Density Tracer Blocks.

\begin{tabular}{cccccccc}
\hline & \multicolumn{3}{c}{ Measurement 1 } & \multicolumn{3}{c}{ Measurement 2 } & Avg +/- \\
\cline { 2 - 7 } Target & $\begin{array}{c}\text { Weight } \\
\text { in Air } \\
\text { SG }\end{array}$ & $\begin{array}{c}\text { Weight } \\
\text { in Water } \\
\text { (gms) }\end{array}$ & $\begin{array}{c}\text { Block } \\
\text { SG }\end{array}$ & $\begin{array}{c}\text { Weight } \\
\text { in Air } \\
\text { (gms) }\end{array}$ & $\begin{array}{c}\text { Weight } \\
\text { in Water } \\
\text { (gms) }\end{array}$ & $\begin{array}{c}\text { Block } \\
\text { SG }\end{array}$ & $\begin{array}{c}\text { trom } \\
\text { SG }\end{array}$ \\
\hline 1.80 & 54.69 & 24.4 & 1.8055 & 54.69 & 24.39 & 1.8050 & -0.005 \\
1.80 & 55.04 & 24.49 & 1.8016 & 55.04 & 24.61 & 1.8087 & -0.005 \\
1.78 & 53.48 & 23.52 & 1.7850 & 53.48 & 23.44 & 1.7803 & -0.003 \\
1.78 & 54.17 & 23.92 & 1.7907 & 54.2 & 23.86 & 1.7864 & -0.009 \\
2.00 & 60.66 & 30.37 & 2.0026 & 60.63 & 30.33 & 2.0010 & -0.002 \\
2.00 & 62.27 & 31.24 & 2.0068 & 62.26 & 31.16 & 2.0019 & -0.004 \\
\hline
\end{tabular}

Another issue that must be considered for the electronic density tracers is abrasion. During use, the outer surfaces of tracer blocks are often chipped away as the blocks pass through the plant circuitry. The loss of volume has minimal effect on conventional density tracers since the blocks are homogenous throughout. However, this is not true for the case of electronic density tracers. Since the embedded transponders are typically of a higher density than the surrounding resin, the tracer blocks become slightly denser as the surface is eroded away. To assess the impact of this problem, the change in block density was calculated using Eq. [3] for different starting densities and for total volume losses of 1, 5, 10 and 20\%. The results summarized in Table 2 indicate that a gain of less than 0.004 SG units is expected for blocks of low density (1.4-1.5 SG) even when up to $20 \%$ of the total block volume is lost. The net increase in density drops to just 0.003 SG units at intermediate densities (1.6-1.7 SG) and is essentially negligible for higher densities (>2.0 SG). These calculations suggest that the loss of block volume caused by normal abrasion/chipping should not be a serious problem for the transponder-based density tracers.

Table 2. Calculated Variation in Density After Loss of Block Volume.

\begin{tabular}{ccccc}
\hline Target & \multicolumn{4}{c}{ Density Tracer SG Versus Percentage Volume Loss } \\
\cline { 2 - 5 } SG & $1 \%$ & $5 \%$ & $10 \%$ & $20 \%$ \\
\hline 1.400 & 1.400 & 1.401 & 1.402 & 1.404 \\
1.500 & 1.500 & 1.501 & 1.502 & 1.504 \\
1.600 & 1.600 & 1.601 & 1.601 & 1.603 \\
1.700 & 1.700 & 1.701 & 1.701 & 1.703 \\
1.800 & 1.800 & 1.800 & 1.801 & 1.802 \\
1.900 & 1.900 & 1.900 & 1.901 & 1.902 \\
2.000 & 2.000 & 2.000 & 2.001 & 2.001 \\
2.100 & 2.100 & 2.100 & 2.100 & 2.101 \\
2.200 & 2.200 & 2.200 & 2.200 & 2.200 \\
\hline
\end{tabular}




\section{Task 4 - Testing and Evaluation}

During the previous reporting period, the components of the EDT technology were tested to determine the operational limits of the system. Static tests conducted previously with stationary blocks indicated that transponder orientation strongly influenced the read range. However, dynamic tests conducted by passing single blocks through the antenna field suggested that the transponders could be identified regardless of orientation provided they were in motion. To test this possibility, a pilot-scale test circuit was constructed using two conveyor belts that constantly circulated the transponder tracers past the reader antenna (Figure 1). The antenna was placed 22 inches above the surface of the belting material. This experimental setup made it possible to determine how the transponder movement and spatial factors (belting material, coal bed, conveyor framing) affected the transponder readings.

Tests conducted with the pilot-scale circuit showed that all of the tracer blocks could be accurately identified as they passed below the antenna regardless of orientation. Therefore, the problem of orientation experienced with the static (stationary) tracers does not appear to be a problem for actual practice. The tests also showed that problems could occur with duplicate readings when two blocks are in close proximity to each other. The reader is designed to forward the transponder ID to the computer only when the ID is different that the one last read. However, if multiple transponders are in the field for a long enough period of time, one can be read and then the other, therefore allowing the first one to be read again. To overcome this problem, each block with require a unique ID number so that it can be compared with the data file log and counted only once by the computer software.

The pilot-scale test circuit also provided a convenient opportunity to evaluate the data acquisition system used to tally the tags and to generate the user report. Two software packages were evaluated for this task. The first program was DASYLab (Data Acquisition System Laboratory). This program is attractive because it has the power to handle several inputs and outputs simultaneously. The problem with this package was that it required very specific formats for reading signals from devices. The TI tag has an identification number that exceeds the character limits of the DASYLab input. Therefore, this package was abandoned in favor of the WinWedge software. WinWedge serves as a pipeline that transfers the data from the reader into another program on the computer. In this case, the data was transferred using DDE (Dynamic Data Exchange) routines to the Excel spreadsheet program for data manipulation and plotting. Figure 2 shows a snapshot of the computer screen after a set of test runs.

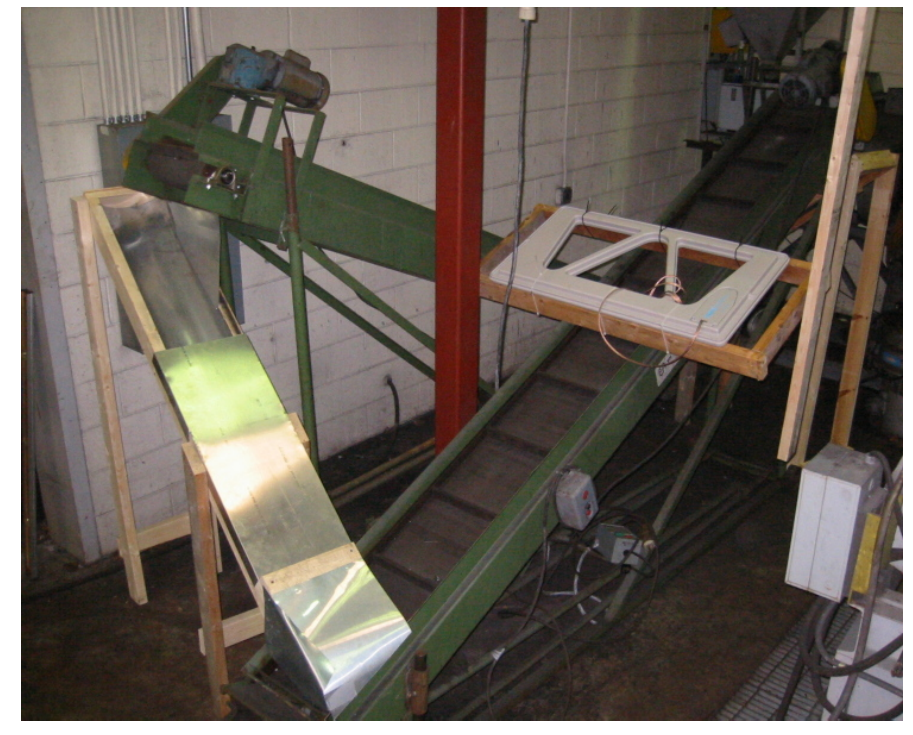

Fig. 1. Pilot-Scale Test Circuit. 


\section{SUMMARY}

Several important activities were completed during the past reporting period. Prototype blocks were manufactured and were found to have a density tolerance that is acceptable for the proposed work (i.e., \pm 0.01 SG units). The change in block density resulting from the loss of volume due to abrasion was also deemed to be within an acceptable range. Pilot-scale tests conducted using twin conveyor belts to simulate an industrial setting indicated that the low-cost TI RFID system is well suited for this particular application. Data obtained from the pilot-scale trials were used to develop an antenna array that can accurately identify tracer tags at a distance of 45-60 cm (18-24 inches) regardless of orientation on a moving conveyor belt.

\section{FUTURE WORK}

During the next reporting period, work is expected to focus on (i) production of a large batch of tracers for testing in an industrial application, (ii) the investigation of different antenna configurations/arrays to extend the capabilities of the system, (iii) continued development of data acquisition system for deployment in industrial environment, and (iv) full-scale field testing at an industrial site.

\section{REFERENCES}

1. DASYLab, “Book 1: User Guide” and "Book 2 Module Reference Guide,” DASYTECH USA, A National Instruments Company, NH, 1991-2001.

2. Scher, Bob, "Antenna Considerations for Low Frequency RFID Applications," http://www.rfidusa.com/rfid_antenna_consid.html.

3. Sorrells, Peter, 2000 “Optimizing Read Range in RFID Systems," EDN Magazine, December 7, 2000, pp. 173-184.

4. Texas Instruments, Series 2000 Reader System, RI-RFM-007B, Reference Guide

5. WinWedge, “RS-232 User Guide,” TAL Technologies, PA, www.taltech.com.

\section{PUBLICATIONS/PRESENTATIONS}

To date, no major publications have resulted from this project. 